\title{
III. Die Bank deutscher Länder, die Konvertibilität und Deutschlands Rückkehr zum europäischen Markt 1952 bis 1955
}

Im Jahre 1952 rückte das Thema der Konvertibilität auf die Tagesordnung der OEEC und der OEEC-Mitgliedsländer. Die Belgisch-Luxemburgische-Wirtschaftsunion ${ }^{1}$ hatte im Verlauf des Jahres 1951 ihre EZU-Quote als Gläubigerin ausgeschöpft und verlangte für ihre EZU-Überschüsse, die über die Quote hinausgingen, im Winter 1951/52 einen höheren Goldanteil, womit sie indirekt Schritte in Richtung Konvertibilität forderte. Auch der Bundeswirtschaftsminister Ludwig Erhard plädierte seit Anfang 1952 für die Aufhebung der Devisenbewirtschaftung. Zur selben Zeit wurden im Vereinigten Königreich, wenn auch vorerst hinter verschlossenen Türen, Pläne für eine Konvertibilitätserklärung und einen EZU-Austritt geschmiedet. Mit diesen Forderungen, Ideen und Plänen mußten sich die OEEC-Länder auseinandersetzen. Als Konsequenz waren die folgenden Jahre von Diskussionen und Verhandlungen über die währungspolitische Kooperation Europas, über die EZU und ihre Zukunft sowie über die Konstruktion eines zukünftigen internationalen Währungssystems geprägt. Erst im Sommer 1955 fanden diese Diskussionen ein vorläufiges Ende. Nachdem die OEEC-Länder schon im Sommer 1954 die EZU modifiziert und leicht gehärtet hatten, hoben sie im folgenden Jahr den Goldanteil für Überschüsse und Defizite von 50 auf 75 Prozent an. Wenn sie sich auch vorerst für die Beibehaltung der EZU entschieden, betonten sie, daß diese Institution bei der nächsten günstigen Gelegenheit aufgelöst werden sollte. Um die dann erreichte Konvertibilität abzufedern, unterzeichneten sie das Europäische Währungsabkommen (EWA) ${ }^{2}$. Mit dem multilateralen System des Zahlungsausgleiches und mit dem nicht-automatischen Kreditsystem des EWA, dem Europäischen Fonds, sollte im Falle der EZU-Auflösung die währungspolitische Kooperation der OEEC-Länder weitergeführt werden. Bis zu diesem Zeitpunkt jedoch sollte das EWA vor allem einen gewissen Schutz vor nationalen Alleingängen bieten. Es enthielt nämlich die Bestimmung, daß die EZU durch das EWA nur auf Antrag von Mitgliedern, die 50 Prozent der EWA-Quoten stellten, ersetzt werden konnte.

Mit den währungspolitischen Beschlüssen der OEEC vom Sommer 1955 war ein Einschnitt in der währungspolitischen Geschichte Europas erreicht. Die

\footnotetext{
${ }^{1}$ Im folgenden werde ich hierfür den Begriff Belgien verwenden.

${ }^{2}$ Das EWA ist abgedruckt in: BGBL 1959 II, S. $293 \mathrm{ff}$.
} 
OEEC-Länder hatten sich damit dem globalen Bretton-Woods-System, das auf Konvertibilität und festen Wechselkursen beruhte, erheblich angenähert. Mehr noch: Da im Verlauf der Jahre einige Länder ihre nationalen Devisenbeschränkungen gelockert hatten ${ }^{3}$ und da das Vereinigte Königreich seit Februar 1955 den Kurs des transferierbaren Pfundes stützte ${ }^{4}$, war in Europa die de-facto-Konvertibilität erreicht ${ }^{5}$. Die Diskussionen und Verhandlungen, die zu diesem Einschnitt führten, sind von Bedeutung für das Verständnis der Nachkriegsgeschichte Europas und der Bundesrepublik. Allerdings steht die historische Erforschung dieser Verhandlungen noch in den Anfängen: Zwar haben Jacob J. Kaplan und Günther Schleiminger eine detaillierte Studie zur EZU und zur financial diplomacy der fünfziger Jahre vorgelegt ${ }^{6}$. Darüber hinaus hat Alan S. Milward in den letzten Jahren die Auseinandersetzungen über die Konvertibilität und über das EWA analysiert ${ }^{7}$. Wenn er dabei auch die konträren Interessenslagen einzelner Staaten herausarbeitet, so ist festzuhalten, daß die nationalen Perspektiven und Entscheidungsprozesse bisher nur unzureichend erforscht wurden ${ }^{8}$. Damit fehlt die Basis für das Verständnis der Konvertibilitätsverhandlungen sowie der Nachkriegsgeschichte Europas und der Bundesrepublik.

In bezug auf die Geschichte Europas erhellen diese Diskussionen und Verhandlungen, wie die EZU von den Mitgliedsländern eingeschätzt wurde, welche Aspekte als unverzichtbar angesehen wurden und wo die Europäer ihre Prioritäten setzten. Darüber hinaus zeigt eine Analyse, wie das mit der EZU geschaffene System funktionierte, welchen Einflüssen es unterlag und wie Einflüsse innerhalb dieses Systems geltend gemacht werden konnten. Ferner wird deutlich, wie die OEECLänder mit der anfänglich noch immer problematischen währungspolitischen $\mathrm{Si}$ tuation in Europa umgingen, wie sie auf Entwicklungen reagierten und wie sie Krisen begegneten: Trotz gesamteuropäisch anwachsender konvertibler Währungsreserven blieb diese Zeit keineswegs krisen- und sorgenfrei'; vielmehr verlief die Ent-

${ }^{3}$ Beispielsweise unterlagen die Deviseninländer in der Bundesrepublik kaum noch Devisenbeschränkungen: Der Handel zum OEEC-Raum war zu 91,3\%, der Handel zum Dollarraum war zu 68\% (mit Einschluß der Einfuhren auf Grund offener Ausschreibungen sogar zu 85\%) liberalisiert. Ähnlich war die Situation für die Schweiz, Belgien und die Niederlande.

${ }^{4}$ Da der Kurs der transferierbaren Pfunde nun in der Bandbreite des offiziellen Wechselkurses gehalten wurde, konnten alle Ausländer ihre Pfund-Guthaben mit einem geringen Diskont in Dollar umtauschen. Dadurch wurde das EZU-System de facto konvertibel, auch wenn die Überschüsse in der EZU nur zum Teil in Gold oder Dollar beglichen wurden.

5 Aus diesem Grund betont z.B. Bordo, Monetary System, S.36, daß das Bretton-Woods-System schon 1955 funktionierte. Dem ist zuzustimmen, obwohl festzuhalten bleibt, daß es weiterhin die EZU und eine europäische Währungskooperation gab. Wie sich diese entwickeln würde, war nicht abzusehen.

${ }^{6}$ Vgl. Kaplan/Schleiminger, EPU.

7 Vgl. Milward, Rescue, S. 345 ff.; ders., Motives; ders., European Monetary Agreement.

${ }^{8}$ Für die Bundesrepublik vgl. Buchheim, Maßnahmen; ders., Wiedereingliederung; ders., Bundesrepublik; Hardach, Rückkehr.

9 Im Juni 1952 beliefen sich die europäischen Reserven auf 8,1 Mrd. \$. Bis Ende 1955 wuchsen sie bis auf 13,8 Mrd \$ an. Vgl. OEEC, 7 th Annual Report (1956), S.104. Hinter diesen aggregierten Zahlen verbergen sich ausgesprochen unterschiedliche Entwicklungen. Vgl. hierzu Seite $144 \mathrm{f}$. 
Abbildung 3: Kumulative Positionen einiger EZU-Mitglieder 1950 bis 1956

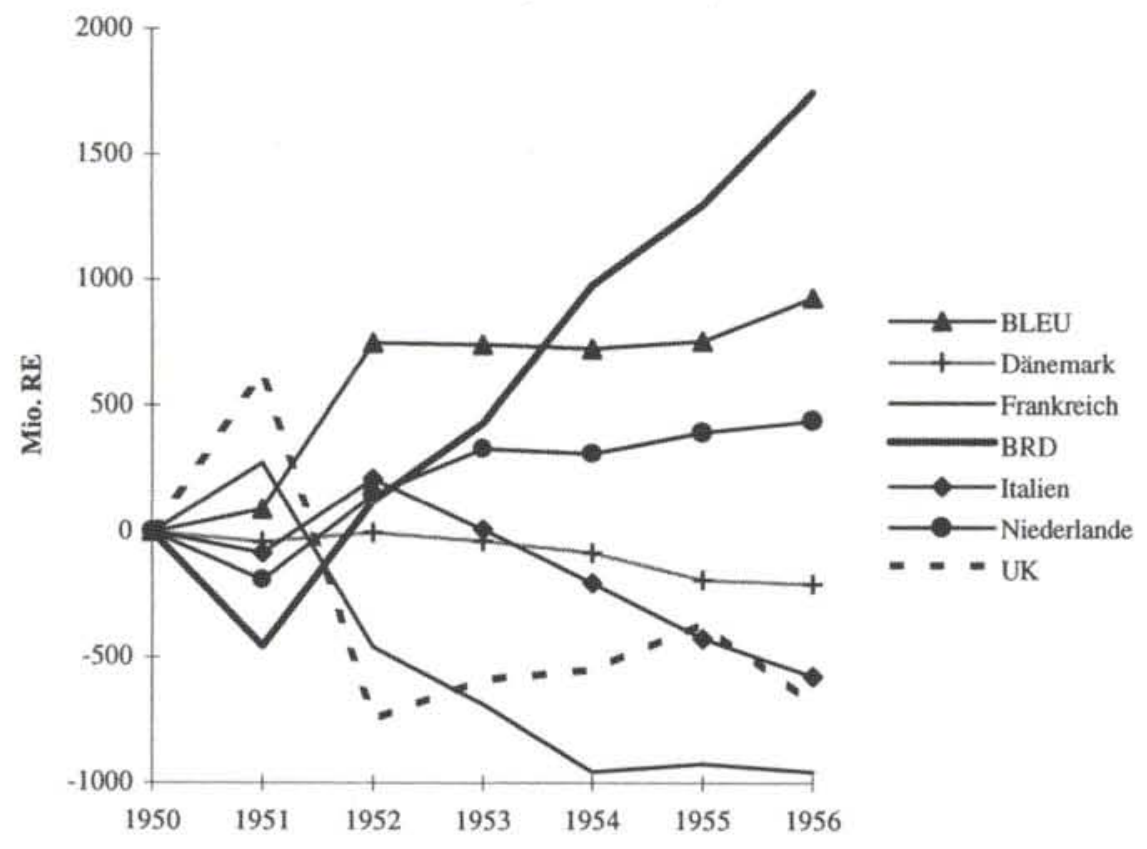

Quelle: BIZ, Annual Reports, div. Ausgaben.

wicklung höchst ungleichgewichtig, und deshalb waren andauernd Krisen zu bewältigen. Wie Abbildung 3 zeigt, hatte die EZU mit extremen Salden zu kämpfen. Während einige Länder - wie die Niederlande und die Bundesrepublik - ihre Zahlungspositionen mit dem Abebben der Koreahausse entscheidend verbesserten, sich zu hartnäckigen Gläubigern der EZU entwickelten und konvertible Reserven akkumulierten, gerieten andere Länder - wie das Vereinigte Königreich und Frankreich - in Zahlungsbilanzschwierigkeiten. Sie akkumulierten EZU-Defizite, hatten ihre EZU-Quoten als Schuldner ausgenutzt und litten durchgängig an ungenügenden Reserven. Vor diesem Hintergrund war das OEEC-Liberalisierungsprogramm permanent bedroht oder gar Rückschritten unterworfen.

Für die Geschichte der Bundesrepublik lassen die Auseinandersetzungen über die Konvertibilität und die währungspolitischen Verhandlungen ihre Handelsorientierungen und die Entwicklung ihrer Handelsmuster in einem anderen Licht erscheinen. Damit wird auch die bisher vorherrschende Interpretation des deutschen Wirtschaftswunders in Teilen modifiziert. Das Wirtschaftswunder wurde und wird oftmals mit dem Modell des export-led-growth in Verbindung gebracht $^{10}$. Dabei wurde und wird die Bundesrepublik in der Tradition von Er-

${ }^{10}$ Zum Modell des export-led-growth vgl. Beckerman, Projecting und kritisch Caves, Growth. Für die deutsche Entwicklung speziell vgl. Michalsky, Export; Hennings, Germany, S.479ff.; Milward, Marshall Plan. Kritisch für die Zeit des Koreabooms: Temin, Koreaboom. 
hards Buch „Deutschlands Rückkehr zum Weltmarkt", das in liberalem Geist schon 1953 diese Rückkehr teils feierte, teils forderte, häufig als außenwirtschaftlich liberales Land gesehen ${ }^{11}$. Da gemäß dieser Interpretation die Bundesrepublik die Rückkehr zum Weltmarkt anstrebte und erreichte, erklärt sich das Wirtschaftswunder nicht zuletzt aus der liberalen deutschen Außenwirtschaftspolitik. Diese Sichtweise ist sicherlich zu einem großen Teil berechtigt. Verfolgt man die bundesrepublikanische Außenwirtschaftspolitik, so ist festzustellen, daß auf diesem Gebiet tatsächlich Erstaunliches geleistet wurde. Seit 1949 wurden Beschränkungen des Handels- und Zahlungsverkehrs kontinuierlich abgebaut. Als Ergebnis war die deutsche Rückkehr zum Weltmarkt im Jahre 1955 abgeschlossen. Zu diesem Zeitpunkt hatte, wie Tabelle 5 zeigt, der deutsche Anteil an den Weltexporten sowie die deutsche Exportquote das Niveau der Weimarer Republik erreicht oder sogar überschritten. Allerdings bleibt dieses Bild unvollständig, was Schatten auf den außenwirtschaftlichen Liberalismus der Bundesrepublik wirft. Während Erhard in seinem Werk die Rückkehr zum Weltmarkt insgesamt propagierte, die Chancen und Erfolge in Lateinamerika, im Mittleren und Fernen Osten ausführlich thematisierte und sich gegen jeden "noch so weitgesteckten Regionalismus" aussprach ${ }^{12}$, verlief die tatsächliche Entwicklung anders. Die regionale Aufgliederung der deutschen Ein- und Ausfuhr in Tabelle 5 zeigt, wie sich bis 1956 die deutschen Handelsmuster entwickelten und verfestigten: Die Dollareinfuhren waren in dieser Zeit von ihrem hohen Nachkriegsniveau auf ein handhabbares Maß zurückgegangen; der amerikanische Markt war insoweit erobert worden, als er nunmehr für die deutsche Wirtschaft die gleiche Bedeutung hatte wie zu Zeiten der Weimarer Republik; der Anteil des Außenhandels, der vor dem Krieg mit Osteuropa abgewickelt wurde, war zum größten Teil auf den EZU-Raum umgelenkt worden; der Export in die übrigen Länder fiel nach einen Anstieg in den Jahren 1949 bis 1954 seit 1955 wieder zurück, denn ihm waren deutliche Grenzen gezogen worden; der EZU-Raum entwickelte sich dagegen zum weitaus wichtigsten Lieferanten und Abnehmer. Wenn auch der Export in den EZU-Raum seit Ende der vierziger bzw. seit Anfang der fünfziger Jahre prozentual zurückgegangen war und der Import aus dem EZURaum, vor allem gegen Ende der Periode, zugunsten der Dollarimporte verlor, so lag er doch erheblich über den Vorkriegszahlen. Bis auf die Verschiebung der Handelsströme von Ost- nach Westeuropa stellt sich somit die Entwicklung des deutschen Außenhandels weitgehend als eine Rekonstruktion der traditionellen deutschen Handelsmuster dar, so wie sie in den zwanziger Jahren bestanden haben. Demnach ist eher von einer Rückkehr zum europäischen Markt zu spre-

\footnotetext{
${ }^{11}$ Vgl. Erhard, Rückkehr. Vgl. als Beispiele dieses Traditionsstranges: Wallich, Triebkräfte; Sohmen, Competition; Klump, Wirtschaftsgeschichte. Auch neuere Forschungen stellen die Rückkehr zum Weltmarkt fest. Sie legen allerdings einen längeren Zeitraum zugrunde. Vgl. Buchheim, Wiedereingliederung; ders., Bundesrepublik; Neebe, Optionen; ders., Überseemärkte; Giersch/Paqué/Schmieding, Miracle.

12 Erhard, Rückkehr, S. 9.
} 
Tabelle 5: Struktur des deutschen Außenhandels 1927, 1936, 1948-1956

Anteilan Export-

Weltex- quote

porten

\begin{tabular}{rcrrrrrrrrr} 
& $\begin{array}{c}\text { porten } \\
\%\end{array}$ & \multicolumn{1}{c}{$\%$} & \multicolumn{1}{c}{$\%$} & \multicolumn{1}{c}{$\%$} & \multicolumn{1}{c}{$\%$} & \multicolumn{1}{c}{$\%$} & \multicolumn{1}{c}{$\%$} & \multicolumn{1}{c}{$\%$} & \multicolumn{1}{c}{$\%$} & \multicolumn{1}{c}{$\%$} \\
\hline 1927 & 7,8 & 13,1 & 60,3 & 7,8 & 18,6 & 13,4 & 50,7 & 17,3 & 14,6 & 17,5 \\
1936 & 9,0 & 5,9 & 60,2 & 4,3 & 17,4 & 18,1 & 55,0 & 6,0 & 16,6 & 22,5 \\
1948 & 1,1 & n.v. & 84,8 & 5,9 & 2,7 & 6,5 & 35,3 & 51,6 & 2,1 & 10,9 \\
1949 & 1,9 & 5,1 & 84,1 & 4,7 & 5,5 & 5,7 & 45,9 & 37,4 & 4,9 & 11,8 \\
1950 & 3,6 & 8,5 & 72,3 & 6,6 & 7,4 & 13,8 & 69,7 & 15,7 & 3,6 & 11,1 \\
1951 & 4,6 & 12,1 & 73,0 & 7,6 & 5,0 & 14,4 & 62,9 & 23,1 & 4,2 & 9,7 \\
1952 & 5,5 & 12,3 & 72,1 & 6,8 & 5,5 & 15,6 & 62,7 & 19,5 & 5,0 & 12,8 \\
1953 & 6,0 & 12,5 & 71,5 & 7,5 & 3,6 & 17,4 & 66,3 & 13,5 & 3,9 & 16,3 \\
1954 & 6,9 & 13,9 & 71,6 & 6,6 & 3,7 & 18,2 & 63,6 & 14,0 & 4,1 & 18,3 \\
1955 & 7,4 & 14,2 & 72,1 & 7,4 & 4,1 & 16,4 & 63,3 & 15,6 & 4,3 & 16,8 \\
1956 & 8,0 & 15,4 & 71,2 & 8,2 & 5,0 & 15,6 & 60,1 & 17,4 & 5,1 & 17,4 \\
\hline
\end{tabular}

Quellen: Sp.1: 1927/36: League of Nations, Review of World Trade; 1949ff.: UN Yearbook of International Trade Statistics.

Sp. 2: 1949: Statistisches Jahrbuch der BRD 1952; 1927, 1936, 1950 ff.: Deutsches Geldund Bankwesen.

Sp. 3-10: 1927/36: Statistisches Jahrbuch für das Deutsche Reich; 1948 ff.: Außenhandel des VWG/der BRD, div. Ausgaben.

Anmerk.: jeweils Reichs- bzw. Bundesgebiet; 1948: VWG.

Importe: cost, insurance, freight; Exporte: free on board; Spezialhandel (reiner Warenverkehr), Herstellung- und Verbrauchsländer. Die Zusammensetzung der Währungsräume unterlag v.a. beim Dollarraum erheblichen Veränderungen. Im Zeitablauf kann er nicht eindeutig geographisch definiert werden. Eingedenk der Tatsache, daß Ab- und Zugänge zum Dollarraum keineswegs zwingend von Dauer waren, sondern vielmehr als adhoc-Lösungen für Zahlungsschwierigkeiten verstanden werden müssen, und um die Vergleichbarkeit sicherzustellen, sind die Zahlungsräume durchgängig wie folgt zusammengesetzt:

Dollar-Raum = U.S. A., Kanada, Philippinen.

EZU-Raum = Mitgliedsländer, abhängige Gebiete und Länder des Sterling-Raumes.

Osteuropa = Albanien, Bulgarien, Finnland, Jugoslawien, Polen, Rumänien, Tschechoslowakei, Ungarn, UdSSR.

chen als von einer Rückkehr zum Weltmarkt ${ }^{13}$. Da die Existenz eines diskriminatorischen Zahlungssystems vor allem den Handelsaustausch innerhalb des Systems fördert, ist diese Entwicklung nicht zuletzt auf die EZU und auf die deutsche Stellungnahme zur EZU bzw. zur Konvertibilitätsfrage zurückzuführen.

Eine Analyse der deutschen Position zur Konvertibilitätsfrage kann aber nicht nur den Blick für die Entwicklung der deutschen Handelsmuster schärfen, sondern sie ist auch notwendig, um die EZU und die Konvertibilitätsverhandlungen

\footnotetext{
${ }^{13}$ Vgl. Geiger, Phoenix.
} 
zu interpretieren. Schließlich erwirtschaftete die Bundesrepublik seit 1951 permanent Überschüsse zum EZU-Raum. Sie wurde daher eine hartnäckige Gläubigerin der EZU, was mit einer entsprechenden Machtposition verbunden war. Darüber hinaus entwickelte sich die Bundesrepublik zu einem wichtigen und unverzichtbaren Markt wie auch zu einem bedeutenden Lieferanten für die anderen OEEC-Länder, so daß sie in den Verhandlungen eine wichtige Rolle spielen konnte - selbst wenn sie formal noch nicht souverän und währungspolitisch nur unter Vorbehalt der Alliierten handlungsfähig war ${ }^{14}$. Innerhalb der Bundesrepublik kam der BdL eine besondere Bedeutung $\mathrm{zu}^{15}$. Die Ausarbeitung einer deutschen Verhandlungsposition und ihre Umsetzung oblag zwar weitgehend der Regierung, in erster Linie dem Wirtschaftsministerium, dem Finanzministerium und dem Marshallplanministerium, wobei sowohl das Bundeskanzleramt als auch das Auswärtige Amt mitsprachen. Die BdL war aber als währungspolitische Beraterin der Regierung und als Devisenbewirtschaftungsinstanz in den Entscheidungsprozeß, die internationalen Verhandlungen sowie die Ausführung der Politik eingebunden. Dabei verfügte sie aus mehreren Gründen über einen großen Einfluß. Zum einen hatte die BdL in den ersten Jahren ihres Bestehens in nationaler wie internationaler Hinsicht an Reputation gewonnen. Zum zweiten vergab sie die Kredite an die EZU, so daß sie erheblichen Einfluß auf deren Fortbestehen und Funktionieren ausüben konnte. Zum dritten war innerhalb der Bundesrepublik die europäische Kooperation und Integration vor allem zwischen Adenauer und Erhard heftig umstritten. Vor dem Hintergrund dieser Auseinandersetzungen kam der Haltung der BdL gestiegene Bedeutung $\mathrm{zu}^{16}$.

Wie bereits beschrieben, nahm Ende 1951 die Außenwirtschaftsstrategie der Zentralbank klare Züge an. Dabei sah sie in der Konvertibilität eine notwendige Grundlage für ein internationales Währungssystem. Allerdings war diese Meinung nur eine prinzipielle Befürwortung der Konvertibilität. Sie blieb unkonkret, war nicht in allen Teilen durchdacht, und über Prioritäten war noch nicht entschieden worden. Andere Elemente innerhalb der Außenwirtschaftsstrategie der Zentralbank - wie z. B. die Befürwortung der OEEC-Liberalisierung - konnten dem Ziel Konvertibilität widersprechen. Als im Winter 1951/52 das Thema der Konvertibilität auf die Tagesordnung rückte, mußte die BdL reagieren und eine konkrete Position ausarbeiten. In den folgenden Kapiteln wird dieser Entscheidungsprozeß analysiert, die Haltung der BdL in nationalen wie internatio-

14 Bis zum Mai 1955 galt das Besatzungsstatut vom 6.3.1951. Das formulierte in Hinsicht auf die Außenwirtschaftspolitik einige Vorbehalte, die allerdings im Verlauf gelockert wurden. 13.8.1951: Übertragung der Führung der Devisenbewirtschaftung auf deutsche Stellen; 13.10.1951: Verzicht auf handelspolitische Vorbehalte; 25.11.1952: Verzicht auf wechselkurspolitische Vorbehalte; 1.6.1953: Ankündigung, daß die ordnungsgemäße Befriedigung der Ansprüche gegen Deutschland nach Inkrafttreten des Londoner Schuldenabkommens nicht mehr kontrolliert wird. Vgl. Kühne, Regelungen, S.138ff., S.198ff. und S. $257 \mathrm{f}$.

15 Das betonen auch Hardach, Rückkehr; Milward, Motives.

${ }^{16}$ Diese Auseinandersetzungen sollten vor allem mit den EWG-Verhandlungen brisant werden. Keime dieser Auseinandersetzungen waren aber schon vorher zu erkennen. Vgl. Küsters, Streit. 
nalen Diskussionen beschrieben sowie ihre Politik in bezug auf unilaterale Devisenbewirtschaftungsmaßnahmen dargestellt. Dabei werde ich weitgehend chronologisch vorgehen.

$\mathrm{Zu}$ Anfang zeigt sich, daß die theoretische und prinzipielle Befürwortung der Konvertibilität weitgehend ein Lippenbekenntnis war. Anläßlich der belgischen Gläubigerkrise und der Erhardschen Kampagne für die Konvertibilität plädierte die BdL für die Beibehaltung des Status quo. In ihren Augen wies der althergebrachte Gold-Devisen-Standard Schwächen auf. Demgegenüber bewertete sie die EZU, die OEEC-Liberalisierung, feste Wechselkurse und eine gewisse Kontrolle der Gläubiger positiv. Wenn die BdL auch zu diesem Zeitpunkt gegen die Konvertibilität optierte, kann man sie jedoch nicht als eine prinzipielle Konvertibilitätsgegnerin bezeichnen. Vielmehr konnte sie wichtige Gründe anführen, die zu diesem Zeitpunkt gegen die Konvertibilität sprachen. Ihres Erachtens waren vor allem die vorerst noch unsicher erscheinende deutsche Zahlungsbilanzsituation und die auf die Bundesrepublik zukommenden Lasten aus der Regelung der Schuldenfrage zu beachten. Wegen dieser Probleme sah sich die Bank vorerst nicht in der Lage, der Konvertibilität zuzustimmen: Eine Konvertibilitätsbefürwortung war ihres Erachtens nicht eine Frage des Wollens, sondern eine Frage des Könnens.

Diese erste Stellungnahme der BdL zur Konvertibilitätsproblematik war also zwiespältig. Darüber hinaus war sie zeitgebunden: Da sich im weiteren Verlauf die deutsche Zahlungsbilanz positiv entwickelte, vergrößerte sich auch der deutsche Handlungsspielraum. Gleichzeitig rückte mit dem Scheitern des Weltbankkredits die Funktion der Konvertibilität, den Kapitalimport zu beleben, in den Vordergrund. Ferner wurden angesichts der deutschen Exportüberschüsse in einer nicht-konvertiblen Welt die bilateralen Kreditierungsverpflichtungen belastender. Auch akzentuierten die deutschen Überschüsse zum EZU-Raum die Kosten des Regionalismus, während die Vorteile zu schwinden schienen. Damit hatte die BdL einen Anlaß, ihre bisherige konvertibilitätskritische und EZU-bejahende Stellungnahme zu modifizieren. In der Analyse, ob und inwieweit das geschah, zeigt sich jedoch, daß die BdL zwiespältig blieb. Weiterhin visierte sie die Konvertibilität als internationales Währungssystem an. Gleichzeitig aber galt ihre Priorität dem Erhalt der EZU und der OEEC-Liberalisierung. Alle Konvertibilitätsbestrebungen wurden diesem Ziel untergeordnet und als zweitrangig eingeschätzt. Trotz positiver deutscher Zahlungsbilanzentwicklung sei die Konvertibilität nicht zu forcieren, denn aus deutschem Interesse müsse, so hieß es innerhalb der BdL, gesamteuropäisch gedacht und gemeinsam vorgegangen werden. Obwohl Kapitalmangel herrschte, war die Konvertibilität nicht wegen ihrer Fähigkeit, Kapitalimporte zu induzieren, zu befürworten. Wenn auch die Kreditierungsverpflichtungen stetig wuchsen, waren die Kredite an die EZU - ganz im Gegensatz zu den Krediten an Verrechnungsländer - vertretbar. Insgesamt sah die BdL die Kosten der EZU als tragbar an, während ihr die Minimierung dieser Kosten unwichtig erschien. Vielmehr war es ihr Anliegen, die EZU trotz deut- 
scher Überschüsse funktions- und arbeitsfähig zu erhalten und dadurch der deutschen Wirtschaft Exportmöglichkeiten zu sichern. Erreichen wollte sie das durch eine Good Creditor Policy, durch einen Ausbau der Liberalisierung und durch einen Abbau der Devisenbeschränkungen.

Wie die BdL versuchte, ihre Position in den Jahren 1953 bis 1955 in Politik umzusetzen, welche Rolle sie bei der Formulierung und Durchsetzung der deutschen Außenwirtschaftspolitik spielte, wird im Anschluß untersucht. Dabei ist zum einen die Einflußnahme der BdL auf die sich nunmehr forcierenden internationalen Verhandlungen sowie die vorbereitenden nationalen Diskussionen zu verfolgen. Zum anderen sind die unilateral verfügten Lockerungen auf dem Gebiet der Devisenbewirtschaftung darzustellen, die sie in Zusammenarbeit mit dem Wirtschafts- und dem Finanzministerium erließ.

In bezug auf die internationalen Verhandlungen wird deutlich, daß die BdL wenn auch der Konvertibilität nicht abgeneigt - durchgängig für die Beibehaltung der OEEC-Liberalisierung und der EZU, möglichst mit britischer Mitgliedschaft, plädierte. Sie befürwortete höchstens eine schrittweise Annäherung an die Konvertibilität. Damit setzte sie sich in Opposition zum Wirtschaftsminister Erhard und zum Vereinigten Königreich, die eine Auflösung der EZU befürworteten. Trotzdem gelang es ihr weitgehend, ihre Meinung gegen diese Opponenten durchzusetzen. Zwar mußte sie dem Vereinigten Königreich im Sommer 1954 aus politischen Gründen entgegenkommen. Doch wenn auch anfänglich befürchtet wurde, daß sich die Bank damit von der EZU abgewandt habe, zeigte sich schon bald, daß sie keineswegs ihr Votum für den Erhalt der europäischen Währungskooperation aufgegeben hatte. In den weiteren Verhandlungen konnte sie es auch durchaus zur Geltung bringen. Einig mit anderen Ressorts, unterstützt durch die einhellige Opposition der kontinentalen EZU-Mitglieder gegenüber den britischen Plänen sowie die abwartende Haltung der USA, profitierend von Erhards Unklarheit und Wankelmut wie auch von der Uneinigkeit der EZUGegner und der wirtschaftlichen Schwäche des Vereinigten Königreiches, konnten Konvertibilitätsinitiativen abgeschlagen, die EZU beibehalten und die mit einer Konvertibilitätserklärung in Europa drohende Handelsdiskriminierung abgewehrt werden. Ferner konnten sich die OEEC-Länder mit dem EWA auf ein währungspolitisches Kooperationsforum einigen, das nach der Konvertibilitätserklärung greifen und bis dahin unilaterale Schritte erschweren sollte.

In bezug auf die unilateral verfügten Lockerungen zeigt sich ein anderes Bild der BdL. Hier wird deutlich, daß sie sich bemühte, die Konvertibilität schrittweise vorzubereiten bzw. sich ihr langsam anzunähern. Mehrere Gründe waren dafür verantwortlich. Zum einen strebte die Bank eine Good Creditor Policy an, da dadurch die EZU erhalten werden konnte. Zum zweiten sah sie es als unabdingbar an, sich auf die Konvertibilität vorzubereiten, da in den Verhandlungen deutlich geworden war, daß der EZU nur noch eine begrenzte Lebensdauer gegönnt war. Darüber hinaus war mit erstarkender Zahlungsbilanzposition die grundsätzliche Kritik an der Konvertibilität in den Hintergrund gerückt, und 
die angestiegenen Reserven legten Lockerungen nahe. Insgesamt zeigt sich also, daß die BdL eine doppelte Strategie verfolgte: Einerseits tat sie alles, um das Fortbestehen der EZU zu ermöglichen; andererseits bereitete sie sich stringent auf die Konvertibilität vor. Dies führte zu dem Nebenprodukt, daß sich die deutschen Handelsmuster in der oben beschriebenen Weise verfestigten.

\section{Die Bank deutscher Länder und die Konvertibilität im Jahre 1951/52}

\section{Die belgische Gläubigerkrise 1951/52}

Die Problemlage

Belgien war das erste europäische Land, das die aus dem Krieg resultierenden wirtschaftlichen Schwierigkeiten überwand. Da es nur geringe Zerstörungen erlitten hatte, konnte es schon Ende 1947 - als einziges europäisches Land - den Produktionsstand der Vorkriegszeit erreichen ${ }^{17}$. Dieses belgische Wirtschaftswunder spiegelte sich auch in der außenwirtschaftlichen Entwicklung Belgiens wider. Da der belgische Export schon früh anlaufen konnte und zudem in Europa eine hohe Nachfrage nach belgischen Produkten bestand, erwirtschaftete Belgien erhebliche Überschüsse mit seinen europäischen Handelspartnern. Das jedoch warf Probleme auf: Sofern es seinen Export nicht einschränken wollte, war es im herrschenden System des Bilateralismus gezwungen, seine Überschüsse zu kreditieren ${ }^{18}$. Das aber stieß auf Unwillen, denn auch Belgien litt an einem Defizit zum Dollarraum, weshalb an Stelle der bilateralen Kreditvergabe eine Konvertierbarkeit der aufgelaufenen Guthaben in Dollar angestrebt wurde. Gleichzeitig wurde an Stelle einer Reglementierung des Außenhandels das Steuerungsinstrument der Währungspolitik befürwortet. Dies würde nicht den Handelsaustausch behindern und sei demnach für ein Land mit traditionell hoher Außenhandelsverflechtung angemessen. Tatsächlich war die Banque Nationale de Belgique eine der wenigen europäischen Zentralbanken, die seit dem Jahre 1944 eine restriktive - oder auch "gesunde“ - Währungspolitik verfolgte ${ }^{19}$, was tendenziell die belgische Überschußposition verstärkte.

Mit seinen Exportüberschüssen und währungspolitischen Ansichten stand Belgien im Gegensatz zu anderen europäischen Ländern. Im Rahmen der Conference for European Economic Co-operation (CEEC) bzw. der OEEC kam es

17 Vgl. Baudhuin, Histoire; de Vries, Benelux; Vandeputte, Geschiedenis; Cassiers, Miracle belge.

${ }^{18}$ Im Jahre 1947 waren die bilateralen Kredite Belgiens an europäische Staaten von 60 Mio. \$ auf 268 Mio. \$ angewachsen. Vgl. BIS, 18 th Annual Report (1947/48), S. 145.

19 Vgl. als Zeitgenossen Dupriez, Politique monetaire, und Baudhuin, Histoire, S. $144 \mathrm{ff}$. In den vierziger und fünfziger Jahren sahen belgische Wirtschaftswissenschaftler diese Politik als eine "gesunde" Währungspolitik an, vgl. Baudhuin, Histoire, S. 150. Heutzutage ist Konsens, daß sie deflationäre Züge aufwies, vgl. z.B. van Rujckeghem, Benelux, S.590 und de Vries, Benelux, S. 31. 
deshalb zu heftigen Auseinandersetzungen. Während die belgischen Vertreter eine Multilateralisierung der Zahlungsabkommen forderten und gegen Kreditierungsverpflichtungen votierten, wurde eine solche Politik in Europa wie in den USA vorerst mit Skepsis gesehen. Die belgischen Handelspartner wollten Ende der vierziger Jahre die aus einer Multilateralisierung möglicherweise resultierenden Goldzahlungen vermeiden ${ }^{20}$. Auch bei den Verhandlungen zur EZU widersetzte sich Belgien im Frühjahr 1950 hohen und automatischen Krediten. Zeitweise blockierte es sogar die Verhandlungen mit seiner Drohung, nicht an der EZU teilzunehmen. Erst als eine niedrigere Quote und dadurch geringere Kreditierungsverpflichtungen sowie weitere Sonderregelungen in Aussicht gestellt wurden, zog Belgien seinen Vorbehalt zurück, so daß das EZU-Abkommen unterzeichnet werden konnte ${ }^{21}$. Allerdings war damit - wie sich bald zeigen sollte - das grundsätzliche Problem nur aufgeschoben, nicht aber gelöst. Tatsächlich entwickelte sich Belgien im Rahmen der EZU zum Gläubiger - zumal im Gefolge des Koreakrieges die Nachfrage nach Rüstungsgütern und damit nach belgischem Stahlerzeugnissen anstieg. Im August 1951 hatte Belgien seine EZU-Quote in Höhe von $331 \mathrm{Mio}$. RE als Gläubiger ausgeschöpft. Damit wurden neue Lösungen notwendig, denn wohlweislich hatte man während der EZU-Verhandlungen die Möglichkeit einer Quotenüberschreitung nicht thematisiert.

Als Belgien im Jahre 1951 seine Quote als Gläubiger ausgeschöpft hatte, verlangte es eine hundertprozentige Abdeckung der Überschüsse, die über die Quote hinausgingen, in Gold. Vor allem die Banque Nationale de Belgique hielt eine weitere Kreditvergabe für unmöglich. Schon der Anstieg auf $17 \mathrm{Mrd}$. belgische Franken (Ende Dezember 1951) hatte ihrer Meinung nach jedes vernünftige $\mathrm{Maß}$ überschritten und zu inflationären Tendenzen geführt ${ }^{22}$. Als im Frühjahr 1952 die Verlängerung der EZU diskutiert wurde, untermauerte Belgien seine Forderung mit der Drohung, seine EZU-Mitgliedschaft zu kündigen ${ }^{23}$. Trotz dieser Ultima ratio stießen aber die belgischen Forderungen in der EZU und bei den anderen EZU-Mitgliedern auf hinhaltenden Widerstand.

Zum ersten wurden prinzipielle Gründe angeführt. Die anderen EZU-Mitglieder machten deutlich, daß es unmöglich sei, einem extremen Gläubiger zu erlauben, sein Dollardefizit mit den über die EZU verdienten Dollars abzudecken. Das würde gegen Charakter und Ziel der EZU verstoßen. Auch würde es die OEEC-Liberalisierung gefährden. Das europäische Dollarproblem würde so nur von einem Land auf ein anderes verschoben, nicht aber gelöst werden. Au-

\footnotetext{
${ }^{20}$ Vgl. Milward, Reconstruction, S.76f. und S.258ff. Vgl. auch Peters-Godts, Politique, S. $234 \mathrm{ff}$. Laurent, America's Ally, analysiert das belgisch-amerikanische Verhältnis in den Jahren 1947 bis 1950. Im Rahmen der CEEC und OEEC war Belgien seines Erachtens „America's Ally“. Allerdings vernachlässigt Laurent dabei die Meinungsvielfalt innerhalb der amerikanischen Regierung. Wahrend er den belgisch-amerikanischen Gleichklang betont, spielt er die belgisch-amerikanischen Meinungsunterschiede herunter.

21 Vgl. hierzu Milward, Reconstruction, S.320ff.; Dickhaus, Provisional; Bloemen, Franken, S.60ff.

22 Vgl. Banque Nationale de Belgique, Rapport 1951, S. $8 \mathrm{ff}$.

${ }^{23}$ Vgl. HAEC, OEEC-TRA 323, Belgian Government, Statement, 26.3.1952.
} 
Berdem könne bei dem wachsenden Dollardefizit keinem europäischen Land zugemutet werden, die für eine Befriedigung der belgischen Ansprüche notwendigen Dollars zur Verfügung zu stellen. Vielmehr - so wurde betont - seien auch die Gläubiger verpflichtet, Maßnahmen zur Korrektur ihres Zahlungsbilanzungleichgewichtes zu ergreifen. Um ein Gleichgewicht innerhalb der EZU zu erreichen, müsse Belgien - so schlußfolgerten beispielsweise der italienische, skandinavische und britische Vertreter im EZU-Direktorium - unter Aufsicht der OEEC eine andere, eine expansivere Politik verfolgen ${ }^{24}$.

Zum zweiten begegneten die belgischen Forderungen objektiven Schwierigkeiten, da sich die EZU zu diesem Zeitpunkt in einer Liquiditätsenge befand. Seit Frühjahr 1951 hatten sich die kumulativen Positionen größerer Mitgliedsländer umgekehrt ${ }^{25}$. Während zuerst die Bundesrepublik, dann die Niederlande Überschüsse erwirtschafteten und so hohe Goldbeträge von der EZU erhielten ${ }^{26}$, wiesen Frankreich und das Vereinigte Königreich Defizite auf. Diese mußten anfänglich - solange die kumulativen Gläubigerpositionen abzubauen waren - zu 50 Prozent in Gold abgegolten werden. Als aber das Vereinigte Königreich und Frankreich im Herbst 1951 kumulative Schuldnerpositionen erreichten, wurden die britischen und französischen Defizite in der ersten Tranche zu 100 Prozent, in der zweiten Tranche zu 80 Prozent usw. kreditiert. Da die britischen und französischen Quoten hoch waren, bedeutete das, daß wenig Gold in die Kassen der EZU floß, während gleichzeitig hohe Beträge ausbezahlt werden mußten. Als Konsequenz sanken die Reserven der EZU von 352 Mio. Dollar im Juni 1951 auf 180 Mio. Dollar im Oktober 195127. Demnach war der Spielraum der EZU, die belgischen Ansprüche zu befriedigen, ausgesprochen gering.

Die Reaktion der BdL: eine supranationale EZU

Die Diskussionen des EZU-Direktoriums über das belgische Problem fanden zu Anfang in Bonn und in Frankfurt nur geringe Aufmerksamkeit. Der deutsche Vertreter im EZU-Direktorium bezog keine pointierte Position und stimmte den vorgeschlagenen ad-hoc-Lösungen wie Aufstockungen der Quote und Abschlagszahlungen $\mathrm{zu}^{28}$. Erst als im Herbst 1951 die Liquiditätsenge der EZU wuchs und als im Frühjahr 1952 die erste EZU-Verlängerung anstand und die

${ }^{24}$ Vgl. HAEC, OEEC-TRA 319, Getz Wold an Cahan, 10.1.1952; Carli an Cahan, Januar 1952; Ellis-Rees an Cahan, 14.1.1952.

${ }^{25}$ Vgl. hierzu Abbildung 3.

${ }^{26}$ Z. B. hatte die Bundesrepublik im Frühjahr 1951 ihre Quote als Schuldner voll ausgeschöpft. Mit den ersten deutschen Überschüssen wurde dieses Defizit abgebaut. Dabei wurden die Überschüsse anfangs zu $80 \%$ in Gold ausbezahlt.

${ }^{27}$ Vgl. EPU, 2nd Annual Report (1951/52), S. 39. Die Trade and Finance Division der OEEC berechnete, daß die EZU-Reserven im Dezember bis auf 61 Mio. \$ schrumpfen könnten. Vgl. HAEC, OEEC-TRA 319, TFD/DI-308, 4.10.1951.

${ }^{28}$ Vgl. HAEC, OEEC-TRA 323, Figgures, An extreme Creditor, TFD/DI-263, 10.9.1951. Vgl. auch BBK, B 330/42, ZBR, Stenogramm, 9.5.1951. Nur im Marshallplan-Ministerium wurde die Frage nach einer Reform der EZU angeschnitten. Vgl. Karl Albrecht, „Kann die Zahlungsunion verbessert werden?", in: Frankfurter Allgemeine Zeitung, Wirtschaftspolitische Beilage, 1.5.1951, S.3; 
belgische Austrittsdrohung vorlag, setzten sich deutsche Stellen intensiver mit der Problematik auseinander. Nun diskutierte auch der Zentralbankrat die belgischen Forderungen ${ }^{29}$. Veit erarbeitete sogar einen Vorschlag, wie die Probleme zu lösen seien ${ }^{30}$. Doch letztendlich lehnte das Zentralbanksystem sowohl den Vorschlag Veits als auch andere imaginative Lösungen, die im Verlauf der Krise ventiliert worden waren, ab. Ein Ausgleich extremer Salden durch entgegengerichtete langfristige Kapitaltransaktionen, eine europäische Investitionsbank, eine regionale Ausweitung der EZU, ein Beitritt der USA, ein neues europäisches Verrechnungsinstitut basierend auf Konvertibilität und auf neuen Dollareinlagen waren seines Erachtens keine sinnvollen Alternativen ${ }^{31}$. Dafür gab es mehrere Motive.

Ein Grund war, daß das Zentralbanksystem auf eine weitere Dollarhilfe der USA spekulierte. Zwar hatten die USA schon im Frühjahr 1951 deutlich gemacht, daß nunmehr die Europäer für das Funktionieren der EZU die Verantwortung übernehmen müßten ${ }^{32}$. Gleichwohl rechnete Emminger noch im März

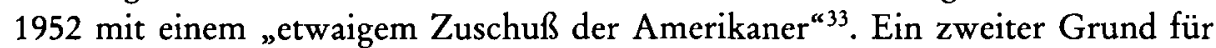
die Ablehnung der Reformvorschläge war die Überzeugung Emmingers, einige Pläne - wie zum Beispiel die Errichtung einer Europäischen Investitionsbank seien währungspolitisch gefährlich ${ }^{34}$, während andere - wie zum Beispiel ein Beitritt der USA oder irgendeine Form der Dollarkonvertibilität - nicht realisierbar seien $^{35}$. Hinzu kam als drittes und wohl wichtigstes Motiv, daß die BdL überzeugt war, solche Vorschläge seien nicht angemessen oder träfen nicht "den Kern der Sache“. Sie seien nicht angemessen, da schon bald "natürliche Aus-

Albrecht, Menschliche, S. 195 ff. Für die Lösungen vgl. BIS, 21 st Annual Report (1951/52), S.231; BIS, 22 nd Annual Report (1952/53), S. 191.

${ }^{29}$ Vgl. BBK, B 330/48, ZBR, Protokoll, 26./27.9.1951 und 10./11.10.1951; BBK, B 330/51, ZBR, Protokoll, 19.12.1951.

${ }^{30} \mathrm{Vgl}$. BBK, B 330/1313, Veit, Wege zur Konvertierbarkeit der europäischen Währungen, 27.12.1951.

${ }^{31}$ Vgl. BAK, B 146/235a-i, II/1, III/2b, Notwendigkeiten und Möglichkeiten für eine Reform der EZU, 16.11.1951; HAEC, OEEC-TRA 310, Cahan, Note, TFD/DI-292, 25.9.1951; Managing Board, The Future of EPU, MBC(51)100, 29.10.1951. Vgl. Emminger, Europäische Zahlungsunion, S. 655 f.; BBK, B 330/3378, Emminger, Stellungnahme zu dem Vorschlag des Herrn Präsidenten Professor Veit, 5.1.1952.

32 Vgl. FRUS 1951 IV, The US Special Representative in Europe (Katz) to the Administrator for Economic Cooperation (Foster), 3. 5.1951; FRUS 1952-54 VI, Report prepared by the Staff Committee of the NAC, 11.3.1952; Report prepared by the Secretary of the NAC, 1.5.1952. Vgl. auch BAK, B 102/6428.1, Diplomatische Vertretung der Bundesrepublik Deutschland, Washington, Bericht über Gespräch mit State Department, 24.4.1952, 30.4.1952.

${ }^{33}$ BBK, B 330/1313, Emminger, Vermerk, Vorschläge des EZU-Direktoriums zur Reform der EZU, 21.3.1952. Vgl. auch BBK, B 330/3478, Emminger, Ergebnisbericht der Sitzung des ERP-Arbeitskreises Intereuropäischer Zahlungsverkehr am 3.1.1952, 16.1.1952. Auch im EZU-Direktorium herrschte dieses Denken vor. Vgl. HAEC, OEEC-TRA 319, Cahan an Selleslags: Preparatory Work for the next meeting of the Managing Board, TFD-387/JFC/vr, 7.11.1951.

${ }^{34} \mathrm{Vgl}$. Emminger, Europäische Zahlungsunion, S. 646.

${ }^{35}$ Vgl. ebenda, S. 655 f., BBK, B 330/3378, Emminger, Stellungnahme zu dem Vorschlag des Herrn Präsidenten Professor Veit, 5.1.1952. 
gleichstendenzen" die momentanen europäischen Ungleichgewichte entschärfen würden. Sie gingen am Kern vorbei, da das Problem bei den extremen Gläubigern, nicht aber bei der EZU läge. Laut BdL wies die EZU bis auf die Diskrepanz der Ein- und Auszahlungen keinen „Konstruktionsfehler" auf. Da sie die Liberalisierung, "die beste Frucht der Pariser Integrationsbemühungen“, ermöglicht habe und da es ohne sie bzw. bei Härtung der EZU zu Rückschritten auf diesem Gebiete kommen müsse, sei sie „unerläßlich“, und demnach unbedingt weitgehend unverändert für mindestens zwei Jahre zu verlängern ${ }^{36}$. Das könne unter den gegebenen Umständen nur auf Kosten der extremen Gläubiger geschehen, denn die anderen EZU-Mitglieder, vor allem die Bundesrepublik, könnten sich höhere Dollarzahlungen nicht erlauben ${ }^{37}$.

Als Konsequenz drängte die BdL darauf, Liberalisierungsverpflichtungen für Gläubiger aufzustellen bzw. den Druck auf die Gläubiger zu verstärken. In diesem Zusammenhang kritisierte sie auch den traditionellen Goldstandard heftig. Emminger stellte heraus, heutzutage müsse die Pflicht zum Ausgleich der Ungleichgewichte „in vielen Fällen primär bei den Überschußländern“ liegen: „Man darf nur nicht vergessen, daß der Goldautomatismus nach dem Ersten Weltkrieg mit dadurch so sehr in Mißkredit geraten ist, daß er zwar mit voller Schärfe auf den Schuldner wirkte, aber - wenigstens nach dem Ersten Weltkrieg - beim Gläubiger völlig versagte. Vielleicht hängt der Kredit der EZU in ähnlicher Weise von einer ,zweiseitigen' Wirksamkeit ab. “38

Um diese "zweiseitige Wirksamkeit" zu erreichen, wollte die BdL sogar die supranationalen Aspekte der EZU verstärkt wissen, denn nur so könnten die Gläubiger kontrolliert werden. Die Bank war also von einer Befürwortung der Konvertibilität, einer Stärkung der Gläubiger und einer Härtung der EZU weit entfernt. Sie stimmte sogar einer Aufweichung der EZU zu ${ }^{39}$.

${ }^{36}$ Emminger, Europäische Zahlungsunion, S. 605 ff., v. a. S. 638 und S. 645; BBK, B 330/1313, Emminger, Vorschläge des EZU-Direktoriums zur Reform der EZU, 21.3.1952.

37 Vgl. BBK, B 330/1313, Emminger, Vorschläge des EZU-Direktoriums zur Reform der EZU, 21.3.1952. Ab Herbst 1951 sanken die deutschen konvertiblen Währungsreserven. Vocke wandte sich deshalb sogar an Schäffer. Vgl. BAK, B 136/7865, Vocke an Schäffer, 23.12.1951; Blücher an Adenauer, 10.1.1952. Vgl. auch BBK, B 330/2043, Vocke an Schäffer, 7.1.1952. Im weiteren Verlauf lockerte sich die Haltung der BdL, ohne jedoch von der grundsätzlichen Linie abzuweichen. Sofern die EZU damit erhalten werden könne und das belgische Problem „in fairer Weise ${ }^{a}$ geregelt würde, stimmte man geringfügigen Dollareinlagen zu. Vgl. BBK, B 330/54, ZBR, Stenogramm, 2.4.1952; BBK, B 330/2015, o. V., Bericht Stand der Verhandlungen zur Fortführung der EZU, 9.5.1952.

${ }^{38}$ Emminger, Europäische Zahlungsunion, S. 646; Otmar Emminger, „Die Etappe der Wahrungsneuordnung", in: Frankfurter Allgemeine Zeitung, Wirtschaftspolitische Beilage, 1.5.1951, S. $5 \mathrm{f}$. Vgl. auch BBK, B 330/3326, Veit, Comité Monetaire Européen, 19.3.1952; BBK, P-A, Vocke, Ansprache auf der Tagung der Deutschen Gruppe der Internationalen Handelskammer, 23.5.1951; BBK, B 330/1313, Emminger, Neuer Kompromißvorschlag für die Stärkung der Dollarreserven der EZU, 16.5.1952.

39 Vgl. BBK, B 330/1313, Emminger, Vorschläge des EZU-Direktoriums zur Reform der EZU, 21.3.1952; Emminger, Neuer Kompromißvorschlag für die Stärkung der Dollarreserven der EZU, 16.5.1952. Die Gläubiger sollten bei Liquiditätsschwierigkeiten der EZU auf einen Teil ihrer Goldzahlungen verzichten. 
$\mathrm{Da}$ die Meinung der BdL weitgehend mit den Ansichten in Bonn ${ }^{40}$ und Paris übereinstimmte und da Belgien seinen Vorbehalt zurückzog, wurde die EZU im Sommer 1952 für ein weiteres Jahr mit nur geringfügigen Modifikationen verlängert $^{41}$. Dabei wurde die belgische Quotenüberschreitung durch zahlreiche Sonderregelungen entschärft. Die EZU zahlte Gold aus, und es wurden bilaterale Abkommen zwischen dem Vereinigten Königreich und Belgien bzw. zwischen Frankreich und Belgien geschlossen. Auch gewährte der IWF seinen ersten stand-by-Kredit an Belgien ${ }^{42}$. Gleichzeitig versuchte die EZU weiterhin, die belgischen Überschüsse mit einer kontinuierlichen Überwachung und mit Empfehlungen von zum Teil diskriminierenden Maßnahmen zu entschärfen. Während die USA und Kanada diese verstärkte Diskriminierung als einen Schritt von der Konvertibilität weg kritisierten, wurde die Richtigkeit einer solchen Politik von der BdL nicht bezweifelt ${ }^{43}$.

\section{Erhards Kampagne für die Konvertibilität}

Erhards Ideen

Am 12.Januar 1952 erläuterte der Wirtschaftsminister Ludwig Erhard auf einer Tagung der evangelischen Akademien Hessen-Nassau seine Wirtschaftspolitik und sprach sich dabei für die Abschaffung der "Devisenzwangswirtschaft" aus $^{44}$. Das war der Beginn einer Erhardschen Kampagne gegen die Devisenbewirtschaftung. In den folgenden Wochen und Monaten griff er dieses Thema in der Öffentlichkeit immer wieder und in immer drastischeren Tönen auf ${ }^{45}$. Auch diskutierte er das Problem der „Devisenzwangswirtschaft" mit der belgischen Regierung. Ebenfalls beauftragte er sein Ministerium mit der Ausarbeitung von

\footnotetext{
${ }^{40}$ Sie stimmte vor allem mit der Meinung des Marshallplan-Ministeriums überein. Demgegenüber stand Erhard auf belgischer Seite. Vgl. BAK, B 102/6428.1, o. V., Vermerk Besprechung Minister Erhard - Belgien, 29.4.1952. Doch trotz seiner Zuständigkeit für "Geld und Kredit ${ }^{*}$ seit Frühjahr 1952 - vgl. Kabinettsprotokolle V (1952), 206. Kabinettssitzung am 11.3.1952, S.157f. - griff Erhard in die entsprechenden Verhandlungen kaum ein. Seine gleichzeitig bzw. nur geringfügig später einsetzende Kampagne gegen die Devisenbewirtschaftung war noch nicht zu Konkreta vorgedrungen.

${ }^{41}$ Die Schuldner wurden durch eine Teilung der ersten Tranche früher zu Goldzahlungen herangezogen. Ferner sollten im Notfalle alle Mitglieder - zuerst die Gläubiger - vorübergehend zur Stärkung der EZU-Reserven beitragen. Vgl. BIS, 23 rd Annual Report (1952/53), S. $190 \mathrm{ff}$.

${ }^{42} \mathrm{Vgl}$. Kaplan/Schleiminger, EPU, S. 144; EPU, 2 nd Annual Report (1951/52), S. $43 \mathrm{ff}$.

${ }^{43}$ Vgl. zu den belgischen Maßnahmen EPU, 2 nd Annual Report (1951/52), S.31 ff.; EPU, 3 rd Annual Report (1952/53), S.23ff. Zur Kritik dieser Maßnahmen vgl. Patterson, Discrimination, S. $93 \mathrm{ff}$.

44 Die Redeunterlagen finden sich in LESt, NE 559.

45 Als Beispiele siehe Hohmann (Hrsg.), Erhard, S.318ff. (6.2.1952: Schweizerisches Institut für Auslandsforschung); Erhard, Aspekte (26.2.1953: Handelshochschule St. Gallen); LESt, NE 1518 (17.4.1952: Deutsch-Belgisch-Luxemburgische Handelskammer); Frankfurter Allgemeine Zeitung, 28.4.1952, S.5, „Erhard auf der Photokina“; Frankfurter Allgemeine Zeitung, 12.5.1952, S.5, „Die Wechselkurse freigeben" (11.5.1952: Außenhandelskammer des DIHT); LESt, NE 858 n (11.10.1952: Italienische Handelskammer).
} 
Positionspapieren, setzte das Thema der Konvertibilität auf die Tagesordnung seines Wissenschaftlichen Beirates und wurde beim Zentralbankrat vorstellig ${ }^{46}$.

Die Gründe, die Erhard für die Abschaffung der „Devisenzwangswirtschaft" anführte, entsprangen seinen ordnungspolitischen Vorstellungen. Er stellte heraus, daß die „Devisenzwangswirtschaft" keine "Ordnungskategorie“" sei. Vielmehr verkörpere sie - als das außenwirtschaftliche Pendant zum „Schlendrian einer Planwirtschaft" und als die logische Konsequenz einer ,inflationären“, auf Vollbeschäftigung ausgerichteten Politik des „billigen Geldes" - die "internationale Unordnung “. Seines Erachtens müßten nunmehr die marktwirtschaftlichen Prinzipien auch im Bereich der Außenwirtschaft zum Tragen kommen. An die Stelle der „Devisenzwangswirtschaft“ - dieses „Symbols alles Bösen“, dieses „Folterinstruments freier Menschen “, dieser „Spottgeburt aus Dreck und Feuer" - müßten nun „gesunde Grundlagen" treten, nämlich die freie Konvertierbarkeit der Währungen. Nur so würde eine „gesunde Politik“ induziert, Vertrauen geschaffen, Kapital angezogen, Handel ermöglicht und die Wirtschaft in Schwung gebracht werden ${ }^{47}$.

Zwar blieb vorerst noch unklar, welche konkreten Konsequenzen Erhard aus seiner prinzipiellen Kritik zog. Weder formulierte er, welche Konvertibilitätsart er anstrebte, noch führte er Modalitäten und Bedingungen einer Konvertibilitätserklärung aus. Auch änderte er des öfteren seine Meinung. Wie er selbst einräumte, lehnte er das existierende System vorerst nur ab, hatte aber „mit der Negation [...] noch keine befriedigende Lösung gefunden ${ }^{\text {"48. }}$. Trotz dieser Vorläufigkeit können aber drei Punkte, auf die Erhard immer wieder zurückkam und die die Diskussion bestimmten, festgehalten werden.

Zum ersten kritisierte Erhard das OEEC-Liberalisierungsprogramm als unzulänglich. Mit Blick auf die französischen und britischen Entliberalisierungsmaßnahmen nannte er das Liberalisierungsprogramm der OEEC zu weich und bezeichnete es als "neckisches Spielchen: ,Hinein in die Liberalisierung, heraus aus der Liberalisierung “' 49 . Deshalb sah Erhard auch das schrittweise Anheben der Liberalisierungsquoten nicht als gangbaren Weg zur Konvertibilität $a^{50}{ }^{50}$ Da das OEEC-Liberalisierungsprogramm unbefriedigend war, hätte er - so führte Erhard aus - in der letzten Zeit "bewußt Zurückhaltung" bei der Erhöhung der deutschen Liberalisierung geübt ${ }^{51}$. Daß das nur eingeschränkt der Wahrheit ent-

\footnotetext{
46 Vgl. BAK, B 102/6428.1, o. V., Vermerk Besprechung Minister Erhard - Belgien, 29.4. 1952; BAK, B 102/56 894, Erhard an Abt. V, 20.5. 1952; Wissenschaftlicher Beirat beim Bundeswirtschaftsministerium, Gutachten vom 27.4.1952, S. 80ff.; BBK, B 330/57, ZBR, Protokoll, 11.6.1952.

47 Für die Zitate siehe Erhard, Aspekte, S. 109 ff.; ders., Wohlstand, S. 343.

${ }^{48}$ Erhard am 6.2.1952 vor dem Schweizerischen Institut für Auslandsforschung, abgedruckt in: Hohmann (Hrsg.), Erhard, S.331.

${ }^{49}$ Erhard, Aspekte, S.111. Im November 1951 hatte das Vereinigte Königreich seinen Liberalisierungssatz von $90 \%$ auf $61 \%$ gesenkt. Im Februar 1952 reduzierte es den Satz sogar auf $46 \%$. Zur gleichen Zeit suspendierte Frankreich seine Liberalisierung. Aufgrund dieser Ereignisse überschrieb die Frankfurter Allgemeine Zeitung, 27.2.1952, S.5, einen Kurzbericht mit dem Titel "Krise in der OEEC".

50 Vgl. Erhard, Aspekte, S. 113.

${ }^{51}$ Ebenda, S. 112.
} 
sprach, war jedoch ein offenes Geheimnis: Im Frühjahr 1952, als die OEEC auf Erhöhung der deutschen Liberalisierung drängte, übte die deutsche Industrie erheblichen Druck auf den Wirtschaftsminister aus und plädierte für Zurückhaltung bei der Erhöhung der deutschen Liberalisierung ${ }^{52}$.

Zum zweiten lehnte Erhard den OEEC-Regionalismus und die EZU prinzipiell ab. Seines Erachtens habe sich dieser grundsätzlich als problematisch erwiesen. Er habe die Bundesrepublik in eine Gläubigerposition geführt, die ihrer strukturellen Situation nicht gerecht würde. Während die Bundesrepublik unter Kapitalarmut leide, würde sie gleichzeitig anderen Ländern über die EZU Kapital zur Verfügung stellen. Darüber hinaus sei die EZU nur beschränkt funktionsfähig. Sie verdecke die wirtschaftspolitischen Fehler anderer Länder „mit einem Schleier der Nächstenliebe“, den EZU-Krediten ${ }^{53}$. Gleichzeitig würden in diesem System die deutschen Überschüsse zu Europa nur zu einer Ansammlung "versteinerter Guthaben" führen, die nicht für den Rohstoffbezug aus anderen Währungsräumen verwertbar seien ${ }^{54}$. Seines Erachtens müsse die EZU aufgesprengt werden, weshalb er an Adenauer schrieb: „Das Ziel der westdeutschen Wirtschaftspolitik muß infolgedessen darauf gerichtet sein, die angeführten institutionellen Hemmungen des zwischenstaatlichen Zahlungs- und Handelsverkehrs auszuräumen. ${ }^{~} 55$ Wenngleich sich Erhard - nach heftiger Kritik aus dem In- und Ausland - beeilte, seine Forderungen nach „Aufsprengung" der EZU als Mißverständnis erscheinen zu lassen ${ }^{56}$, und er im folgenden oftmals lediglich eine Reform der EZU forderte, blieb sein Unwillen über die EZU ein vorherrschendes Element. Zweifel über seinen Reformwillen blieben deshalb bestehen ${ }^{57}$.

Ein dritter Punkt, auf den Erhard immer wieder zu sprechen kam und der seines Erachtens eng mit der Konvertibilität zusammenhing, waren die Wechselkurse. Er stellte heraus, daß Konvertibilität mit der "Starrheit der Wechselkurse“ unvereinbar sei $^{58}$. Was er damit meinte und was er als Konsequenz anstrebte, blieb aber undeutlich und changierte ${ }^{59}$. Manchmal kritisierte er die „falschen“ oder

52 Vgl. die Berichterstattung in der Frankfurter Allgemeine Zeitung v. a. Februar 1952ff. Vgl. auch BBK, B 330/2055, Direktorium, Protokoll, 13.3.1952; BAK, B 146/525, von Mangoldt an BMM, 9.11.1951.

${ }^{53}$ Erhard, Aspekte, S. 112.

${ }^{54}$ Ebenda, S. 122. Die "versteinerten Guthaben“ beliefen sich im Frühjahr 1952 auf 100 Mio. RE.

${ }^{55}$ Erhard an Adenauer, 6.11.1952, zit. in BAK, B 102/56905, I A 1, Arbeitsunterlage, 2183/53, 3.8.1953.

56 Vgl. Erhard, Aspekte, S.111.

57 Vgl. BBK, NL Emminger, Ausländische Stellungnahmen zur Konvertibilität, von Mangoldt an Erhard, 25.10.1952; Kabinettsprotokolle VI (1953), 7. Kabinettssitzung am 10.11.1953, S. 510.

${ }^{58} \mathrm{Vgl}$. die Rede Erhards vor dem Schweizerischen Institut für Auslandsforschung am 6.2.1952, abgedruckt in: Hohmann (Hrsg.), Erhard, S.321.

$59 \mathrm{Vgl}$. BAK, B 146/482, Gerbaulet, Vermerk, 25.11.1952. Gerbaulet vom BMM bezeichnete das als die „vorübergehenden Meinungen des Herrn Minister Erhard “. Festzuhalten ist, daß Erhard keine Zuständigkeit auf dem Gebiet der Wechselkurse hatte. Unter dem Besatzungsstatut vom 6.3.1951 hatten sich die Alliierten die Kontrolle des deutschen Wechselkurses vorbehalten. Erst am 25.11.1952 verzichtete die Alliierte Hohe Kommission auf diesen Vorbehalt. Vgl. Kühne, Regelungen, S. 198. Deshalb konnte Erhard lediglich mit einem ballon d'essai eine Diskussion eröff- 
„unrealistischen" Wechselkurse und legte damit nahe, daß europäische Wechselkursanpassungen notwendig seien ${ }^{60}$. Dann wieder griff er die „starren“, „staatlich fixierten“, „willkürlichen“, „zementierten“ Wechselkurse an, plädierte für „freie " Wechselkurse und schien so ein System schwankender Wechselkurse oder ein „Herantasten" an die richtige Wechselkursrelation anzustreben ${ }^{61}$. Manchmal befürwortete er auch einen Mittelweg wie zum Beispiel relativ stabile Wechselkurse in einer Bandbreite von $+/-5$ Prozent $^{62}$. Aber auch in anderer Hinsicht blieben Fragen offen. So führte Erhard nicht aus, welche Währungen abgewertet werden sollten ${ }^{63}$. Genausowenig thematisierte er die mit einer Wechselkursfreigabe unter Umständen verbundenen Probleme für die $\mathrm{DM}^{64}$. Schließlich löste er den von ihm selbst geschaffenen Widerspruch nicht auf, wie eine Konvertibilität bei flexiblen Kursen die von ihm angestrebte "gesunde“ Politik erzwingen sollte.

\section{Die Reaktion der BdL: kein Chaos}

Da die Konvertibilität mit Erhards Kampagne im Frühjahr 1952 in Deutschland ein öffentlich diskutiertes Thema wurde ${ }^{65}$, mußte auch die BdL Stellung beziehen. Verschiedene Abteilungen arbeiteten Grundsatzpapiere zu den Fragen Devisenbewirtschaftung und Konvertibilität aus. Dabei zeigten sich deutliche Kontraste zwischen Erhard und der BdL hinsichtlich der Art und Weise der Diskussion, der zugrundeliegenden Ordnungsvorstellungen und der konkreten Politikschritte.

Zum ersten war das Zentralbanksystem keineswegs einverstanden mit der Erhardschen Kampagne. Ganz im Gegenteil: Es war ausgesprochen verärgert. Emminger sah diesen Feldzug als „bedenklich“ und „geradezu gefährlich“ an. Die Anprangerung des Devisenbewirtschaftungssystems als „unmoralisch“ führe dazu, daß Verstöße als „Kavaliersdelikte“ verstanden würden. Auch brächten die öffentlichen Äußerungen Erhards die Bundesrepublik in internationalen Verhandlungen „in größte Schwierigkeiten“, denn sie würden im Ausland den Eindruck erwekken, alle Zahlungsbilanzschwierigkeiten könnten „spielend gelöst werden ${ }^{\text {“66 }}$. Das

nen. Daß die Alliierten, zumindest die Briten, noch Ende 1951 diesen Vorbehalt, „however embarrassing", wahrnehmen wollten, ergibt sich aus PRO, T 236/4579, Rootham an Stevenson, 3.12.1951.

${ }^{60}$ Erhard, Aspekte, S. 112.

${ }^{61}$ Erhard vor dem Schweizerischen Institut für Auslandsforschung am 6.2.1952, abgedruckt in: Hohmann (Hrsg.), Erhard, S.331; Erhard, Aspekte, S.122; BBK, B 330/57, ZBR, Stenogramm, 11.6.1952.

62 Vgl. BAK, B 102/25879, Drechsler, Vermerk zu Wechselkursen und zum Exposé vom 6.12.1952, 12.12.1952; BBK, B 330/3171, BMWi, Abt. I, Konvertibilität der Währungen, 6.12.1952; BMWi, Abt. I, Konvertibilität der Währungen, 10.3.1953.

${ }^{63}$ Eine Abwertung der DM zum Dollar befürwortete Erhard nicht. Vgl. BBK, B 330/57, ZBR, Stenogramm, 11.6.1952.

${ }^{64}$ Von Mangoldt wies Erhard darauf hin, daß fluktuierende Kurse möglicherweise zu einer Kursverbesserung der DM und somit zu einer Erschwerung der deutschen Exporte führen könnten. Vgl. LESt, I 4) 52, von Mangoldt an Erhard, 24.7.1952.

${ }^{65} \mathrm{Vgl}$. die Berichterstattung in der Frankfurter Allgemeinen Zeitung, Mai $1952 \mathrm{ff}$.

66 BBK, NL Emminger, Konvertibilität/Abbau der Devisenzwangswirtschaft I 1949-52, Emminger, Abbau der Devisen-Zwangswirtschaft, 15.5.1952; BBK, B 330/56, ZBR, Stenogramm, 30.4.1952. 
war aber nicht die Einschätzung der Bank, weshalb sie das Thema der Konvertibilität und der Devisenbewirtschaftung vorerst von der Tagesordnung verbannen wollte. Ferner war sie der Ansicht, die deutsche Seite dürfe die Diskussion keinesfalls vorantreiben ${ }^{67}$.

Abgesehen von diesen Vorgehensfragen brachte die BdL den Erhardschen Anpreisungen, daß die Konvertibilität der Wirtschaft Impulse gebe, Kapital anzöge und Vertrauen schüfe, beträchtliche Skepsis entgegen ${ }^{68}$. Zwar räumte sie ein, daß ein freier Welthandel „uns voraussichtlich wirtschaftliche Ausweitungsmöglichkeiten geben [würde]". Sie hob aber in diesem Zusammenhang sogleich die Bedeutung des europäischen Marktes hervor, der zuerst „vereinheitlicht" werden müsse $^{69}$. Ebenfalls äußerte die BdL erhebliche Zweifel bezüglich der Erhardschen Ordnungsvorstellungen. Die Abschaffung der Devisenbewirtschaftung würde keine Ordnung bringen, sondern vielmehr "den Sprung in das Chaos" bedeuten. Das nationale Devisenaufkommen würde "einfach verplempert ${ }^{\text {" } 70}$. Zwar lehnte die Bank weder marktwirtschaftliche Prinzipien grundsätzlich ab, noch mißbilligte sie die Währungspolitik als Steuerungselement. Sie führte aber aus, daß die Devisenbewirtschaftung notwendig sei, um die Außenwirtschaft in "geordneten Bahnen" aufrechtzuerhalten. Außerdem diene die Devisenbewirtschaftung dazu, "wirtschaftliche Angriffe abzuwehren " und den Außenhandel zu lenken ${ }^{71}$.

In bezug auf die konkreten Politikschritte war vor allem die Frage der OEECLiberalisierung ein Zankapfel zwischen Erhard und der $\mathrm{BdL}^{72}$. Während Erhard bei der Erhöhung der Liberalisierung zögerte, stellte die BdL den Abbau der mengenmäßigen Beschränkungen in den Vordergrund. Da ein Abbau der Devisenkontrolle hinsichtlich des Kapitalverkehrs nicht ins Auge gefaßt werden könne, würde Konvertibilität nichts anderes als die möglichst weitgehende Liberalisierung aller sicht- und unsichtbaren Einfuhren bedeuten. Da weiterhin die Voraussetzungen für eine Liberalisierung zum Dollarraum wie ausreichende Währungsreserven und Gleichgewicht der Dollarbilanz „für die nächste Zukunft kaum herstellbar" seien, sei - „praktisch und konkret gesprochen“ - der geforderte Abbau der Devisenbewirtschaftung mit „möglichst raschen Fortschritten

\footnotetext{
${ }^{67} \mathrm{Vgl}$. BBK, B 330/2043, Vocke an Schäffer/Erhard, 4.8.1952. Vgl. auch BBK, B 330/56, ZBR, Stenogramm, 30.4.1952.

68 Vgl. BBK, NL Emminger, Konvertibilität/Abbau der Devisenzwangswirtschaft I 1949-52, Emminger, Maßnahmen zur Wiederherstellung der Konvertibilität der Währung, o.D.; BAK, B 102/ 6428, Elson, Niederschrift über die Ressortbesprechung am 1.7.1952, 3.7.1952; BBK, B 330/57, ZBR, Stenogramm, 11.6.1952.

${ }^{69}$ BBK, B 330/57, 6c (Devisenbewirtschaftung), Vermerk, 30.5.1952.

${ }^{70}$ BBK, B 330/57, Wilhelm, Vermerk, 27.5.1952. Vgl. auch BBK, B 330/2012: Vocke an Erhard, 11.12.1952.

71 BBK, B 330/57, 6c (Devisenbewirtschaftung), Vermerk, 30.5.1952.

72 Das war auch ein Streitpunkt zwischen Wirtschaftsministerium einerseits und Marshallplan-Ministerium, der deutschen OEEC-Delegation, der OEEC andererseits. Vgl. BAK, B 146/526, Germania Paris an BMM/Erhard/Niklaß/Vocke, 6.3.1952; BAK, B 102/6428, Elson, Niederschrift über die Ressortbesprechung am 1.7.1952, 3.7.1952; BAK, B 146/527, Elson, Vermerk, 22.6.1952; $\mathrm{BMW}$ an BMM, 24.7.1952.
} 
in der Liberalisierung gegenüber dem EZU-Raum“ identisch" ${ }^{73}$. Zwar sah die BdL durchaus die Probleme dieser Politik. Sie pflichtete Erhard bei, daß das OEECLiberalisierungsprogramm ungenügend sei und als "Liberalisierungsschaukel“ funktionieren würde. Um dem abzuhelfen, schlug sie - wie schon bei der Belgien-Krise - einen stärkeren Einfluß der europäischen Organisationen vor $^{74}$. Gleichzeitig aber forderte sie eine Erhöhung der deutschen Liberalisierung, zum Teil verlangte sie sogar eine Erhöhung des Liberalisierungssatzes auf 100 Pro$z^{2 e n t^{75}}$. Der vornehmliche Grund hierfür lag darin, daß die BdL das System der Devisenkontrollen erhalten wollte. Direktoriumsmitglied Wolf verwahrte sich in einer Ressortbesprechung vehement dagegen, „daß die Devisenpolitik vor den Wagen der Handelspolitik gespannt werde ${ }^{\alpha 76}$.

Auch in bezug auf den Regionalismus teilte das Zentralbanksystem Erhards Anschauungen nicht. Zwar pflichteten einige Mitglieder des Zentralbankrates Erhards negativer Einschätzung der OEEC/EZU bei. Pfleiderer bezeichnete in einer Sitzung des Zentralbankrates die Pariser Gremien als „Kaffeekränzchen“, während Bernard fragte, ob nicht „einige Dinge ohne Fühlungnahme mit anderen Ländern erledigt werden können“. Insgesamt aber befürwortete der Zentralbankrat den Weg über die EZU: Gemeinsam mit allen anderen EZU-Mitgliedsländern müsse langsam und schrittweise vorgegangen werden ${ }^{77}$. Dafür sprachen „politische und taktische "Gründe wie z. B. die europäische Integration, vor allem der Schumanplan und die Verteidigungsgemeinschaft ${ }^{78}$. Aber auch die wirtschaftliche Situation war maßgebend, denn schließlich - so betonte Emminger - umfasse der EZU-Raum "rund $3 / 4$ des auswärtigen Zahlungsverkehrs der Bundesrepublik". Dieser dürfe nicht behindert werden - zumal für die Bundesrepublik nicht die Konvertibilität der DM, sondern vielmehr die Konvertibilität der anderen Währungen das Kardinalproblem war ${ }^{79}$. Insgesamt sah die BdL regionale Organisationsformen als unverzichtbar und notwendig an. Der Regionalismus sei keinesfalls abzubauen oder gar aufzusprengen. Wegen der amerikanischen Zollpolitik und der Schwierigkeiten des amerikanischen Marktes könne die deutsche Ausfuhr in den Dollarraum nicht

${ }^{73}$ BBK, B 330/57, Emminger, Thesen zur Frage des Abbaus der Devisenzwangswirtschaft, Juni 1952.

${ }^{74}$ BBK, NL Emminger Konvertibilität/Abbau der Devisenzwangswirtschaft I 1949-52, Emminger, Konvertibilität und Liberalisierung, 15.12.1952.

75 Vgl. BBK, B 330/2043, Vocke an Schäffer/Erhard, 4.8.1952; BAK, B 102/6428, Elson, Niederschrift über die Ressortbesprechung am 1.7.1952, 3.7.1952. Wie interne Dokumente zeigen, ist dieses Plädoyer auch als Gegenposition zu Erhard zu verstehen. Vgl. BBK, NL Emminger, Konvertibilität/Abbau der Devisenzwangswirtschaft I 1949-52, Emminger, Bemerkungen hinsichtlich der deutschen Stellungnahme zur Liberalisierung und Konvertibilität, 10.9. 1952; Emminger, Konvertibilität und Liberalisierung, 15.12.1952.

${ }^{76}$ BAK, B 102/6428, Elson, Niederschrift über die Ressortbesprechung am 1.7.1952, 3.7.1952.

${ }_{77}$ BBK, B 330/57, ZBR, Stenogramm, 11.6.1952.

78 Ebenda; BBK, NL Emminger Konvertibilität/Abbau der Devisenzwangswirtschaft I 1949-52, Emminger, Abbau der Devisen-Zwangswirtschaft, 15.5.1952.

79 BBK, NL Emminger, Konvertibilität/Abbau der Devisenzwangswirtschaft I 1949-52, Emminger, Bemerkungen hinsichtlich der deutschen Stellungnahme zur Liberalisierung und Konvertibilität, 10.9.1952; BBK, B 330/57, Emminger, Thesen zur Frage des Abbaus der Devisenzwangswirtschaft, Juni 1952. 
gesteigert werden. Hier müsse weiterhin mit Einfuhrrestriktionen operiert werden. Es müsse strikt zwischen Dollarraum und EZU-Raum getrennt werden ${ }^{80}$.

Die Frage der Wechselkurspolitik führte ebenfalls zu einer „ernsthafteren Meinungsverschiedenheit ${ }^{*} z$ wischen BdL und Wirtschaftsminister ${ }^{81}$. Die BdL lehnte Korrekturen des Wechselkurses und jede Art der Flexibilität strikt und rundheraus ab. Zwar hatte der Zentralbankrat Anfang 1952 der Schaffung von sogenannten Einfuhranrechten zugestimmt und dadurch sein Placet zu einem gespaltenen Wechselkurs bzw. zu einer umgrenzten und selektiven Abwertung zum Dollarraum gegeben ${ }^{82}$. Gleichwohl sah er dieses nur als eine vorübergehende Notmaßnahme an und wollte sie in einem engen und kontrollierbaren Rahmen halten ${ }^{83}$. Die Gründe für die Ablehnung der Wechselkursflexibilität waren zum einen politischer Natur. Bernard wies darauf hin, daß der Beitritt zum IWF gewisse Verpflichtungen mit sich bringe ${ }^{84}$. Emminger warnte, die USA würden flexible Kurse nicht billigen, und das könnte zu Retorsionsmaßnahmen Anlaß geben ${ }^{85}$. Auch würden flexible Wechselkurse die europäische Integration - sei es in der EGKS/EVG-Form, sei es in der EZU-Form - gefährden ${ }^{86}$. Zum zweiten spiel-

${ }^{\text {so }}$ Vgl. BBK, NL Emminger, Konvertibilität/Abbau der Devisenzwangswirtschaft I 1949-52, Emminger, Abbau der Devisen-Zwangswirtschaft, 15.5.1952.

${ }^{81}$ BBK, B 330/2012, Vocke an Erhard, 19.3.1953. Flexible Wechselkurse trafen auch auf Skepsis bei der deutschen EZU-Delegation. Vgl. LESt, I 4) 52, von Mangoldt an Erhard, 24.7.1952. Vom BMM hingegen wurden sie zeitweise befürwortet. Vgl. BAK, B 102/11 579.1, Albrecht, Vermerk zum Exposé des BMWi über Konvertibilität, 9.12.1952; BBK, B 330/2055, Direktorium, Protokoll, 4.12.1952.

${ }^{82}$ Vgl. BBK, B 330/53, ZBR, Protokoll, 21./22.2.1952; BBK, B 330/54, ZBR, Protokoll, $19 . /$ 20.3.1952; BBK, B 330/2055, Direktorium, Protokoll, 14.2.1952. Die Einfuhranrechte sollten die Ausfuhr in den Dollarraum fördern. Sie funktionierten folgendermaßen: Die Exporteure hatten ein Anrecht auf 40\% ihrer Dollarerlöse. Diese Anrechte konnten sie innerhalb von drei Monaten entweder für den Import bestimmter Waren verwenden oder veräußern. Die Anrechte wurden Mitte April mit einem Aufschlag von 20 bis 20,75\% gehandelt. Mitte Mai belief sich der Aufschlag auf 13 bis 14,5\%. Vgl. Frankfurter Allgemeine Zeitung, 22.4.1952 und 20.5. 1952. Der Exporteur erhielt somit Mitte April für 1 \$ anstatt 4,20 DM nun 4,54 DM, im Mai nur noch 4,41 DM. Der Importeur mußte - sofern er vollkommen auf Einfuhranrechte zurückgriff - im April einen Kurs von 5,04 DM, im Mai von 4,75 DM in Anschlag bringen. Die Liste der Waren, die mit Einfuhranrechten eingeführt werden konnten, ist in BAnz 69/52 vom 8.4.1952 enthalten. Anfangs umfaßte sie nur wenige Güter, sie wurde aber im August 1952 erheblich erweitert. Vgl. BAnz 147/52 vom 1.8.1952.

${ }^{83}$ Die Einfuhranrechte waren im Zentralbankrat umstritten. Vgl. BBK, B 330/57, ZBR, Stenogramm, 11.6. 1952; BoE, OV 34/38, Macdonald, Report on a visit to Germany, 23.7.1952. Die Kritiker wurden durch die negative Stellungnahme der Alliierten Hohen Kommission - vgl. BBK, B 330/ 2055, Direktorium, Protokoll, 18.6.1952 - und die Anfrage der Federal Reserve Bank, ob mit den Einfuhranrechten ein gespaltener Kurs eingeführt worden wäre - vgl. BBK, B 330/58, ZBR, Protokoll, 9.7.1952 -, gestärkt. Auch der IWF monierte die Anrechte als Restriktion. Vgl. IMF, 4 th Annual Report on Exchange Restrictions, S.162f.; BBK, B 330/1087, Donner an BMWi, 25.2.1953.

${ }^{84}$ Vgl. BBK, B 330/57, ZBR, Stenogramm, 11.6.1952.

${ }^{85}$ Vgl. BAK, B 146/565, Schleiminger, Behandlung der Frage der DM-Konvertibilität im Interministeriellen Wirtschaftsausschuß, 19.5.1952.

${ }^{86}$ Vgl. BBK, NL Emminger, Konvertibilität/Abbau der Devisenzwangswirtschaft I 1949-52, Emminger, Bemerkungen hinsichtlich der deutschen Stellungnahme zur Liberalisierung und Konvertibilität, 10.9.1952. 
ten wirtschaftliche Motive eine Rolle. Flexible Wechselkurse - so führten Vocke und Emminger aus - würden den Außenhandel stören und Unruhe verbreiten. Die daraus resultierende Spekulation könnte einen stärkeren Einsatz von Währungsreserven notwendig machen ${ }^{87}$. Das würde auch bei relativ stabilen Wechselkursen, wie sie das Wirtschaftsministerium zeitweise befürwortete, der Fall $\operatorname{sein}^{88}$. Wichtiger aber war, daß die BdL starke und prinzipielle Vorbehalte gegen jede Form von Abwertung zum Dollar hegte. Wie schon 1949 und 1950/51 so meinte sie auch 1952, daß eine Abwertung nur zu einer „schweren Krise“ führen würde ${ }^{89}$. Ihres Erachtens war die Dollarlücke güterbedingt und strukturell. Deshalb könne durch eine Abwertung und niedrigere Exportpreise ein Ausgleich nicht erreicht werden. Vor allem könne „die Hürde der Hochzollpolitik“ dadurch nicht übersprungen werden ${ }^{90}$. Darüber hinaus würde die Konkurrenzfähigkeit der Exportindustrie, die Dollarrohstoffe benötige, beeinträchtigt, denn eine Abwertung würde die terms of trade deutlich verschlechtern ${ }^{91}$. Zum Dollarraum seien weiterhin "nicht-marktwirtschaftliche“ Methoden anzuwenden. Emminger führte aus: „Wenn schon die Dollarlücke in der Hauptsache nicht durch Steigerung der Ausfuhrerlöse, sondern nur durch Einfuhrbeschränkung geschlossen werden kann, dann ist mindestens in der gegenwärtigen Lage die Beschränkung der hereinzunehmenden ,Dollarwaren' durch Einfuhrkontingente der Beschränkung über eine allgemeine Verteuerung lebenswichtiger Nahrungsmittel und Rohstoffe eindeutig vorzuziehen." ${ }^{\text {92 }}$

Als Erhard Anfang 1952 seine Konvertibilitätskampagne startete, kam der BdL die Rolle eines Gegenspielers zu. Statt Konvertibilität bei flexiblen Wechselkursen befürwortete sie die weitere OEEC-Liberalisierung, den Regionalismus und feste Wechselkurse. An einer Verwirklichung der marktwirtschaftlichen Prinzipien in der Außenwirtschaft war ihr nicht gelegen.

${ }^{87}$ Vgl. BBK, NL Emminger, Konvertibilität/Abbau der Devisenzwangswirtschaft I 1949-52, Emminger, Abbau der Devisen-Zwangswirtschaft, 15.5.1952; BBK, B 330/2012, Vocke an Erhard, 19.3.1953.

${ }_{88}$ Vgl. BBK, B 330/2012, Vocke an Erhard, 11.12.1952.

${ }^{89}$ BBK, B 330/2043, Vocke an Schäffer, 4.8.1952. Vgl. auch BBK, B 330/2012, Vocke an Erhard, 11.12.1952.

90 BAK, B 146/565, Schleiminger, Behandlung der Frage der DM-Konvertibilität im Interministeriellen Wirtschaftsausschuß, 19.5.1952; BBK, B 330/2012, Vocke an Erhard, 11.12.1952; BBK, B 330/ 57, ZBR, Stenogramm, 11.6.1952.

${ }^{91}$ Vgl. BBK, B 330/57, ZBR, Stenogramm, 11.6. 1952; BBK, NL Emminger, Konvertibilität/Abbau der Devisenzwangswirtschaft I 1949-52, Emminger, Abbau der Devisen-Zwangswirtschaft, 15.5.1952.

92 BBK, NL Emminger, Konvertibilität/Abbau der Devisenzwangswirtschaft I 1949-52, Emminger, Abbau der Devisen-Zwangswirtschaft, 15.5.1952. Vgl. auch BAK, B 146/565, Schleiminger, Behandlung der Frage der DM-Konvertibilität im Interministeriellen Wirtschaftsausschuß, 19.5.1952. 


\section{Konvertibilität: „eine Frage des Könnens“}

Wenn sich auch die BdL in Reaktion auf die belgischen Forderungen und die Erhardsche Kampagne als eine Befürworterin des Status quo entpuppte, so ist damit ihre Haltung zur Konvertibilität noch nicht vollständig erfaßt. Vielmehr führte die BdL für die Ablehnung der Konvertibilität auch Motive an, die in diesem Moment eine Befürwortung der Konvertibilität erschweren oder gar unmöglich machen würden. Wie die Abteilung Devisenbewirtschaftung ausführte, waren die Zahlungsbilanzsituation, der Verteidigungsbeitrag und die Belastung aus der Schuldenregelung zu bedenken. Deshalb sei die Konvertibilität eine „Frage des Könnens, nicht des Wollens" ${ }^{* 33}$.

\section{Die Zahlungsbilanzentwicklung}

Wie Tabelle 6 zeigt, war die deutsche Zahlungsbilanz- und Reservenentwicklung im Jahre 1952 ausgesprochen positiv. Allerdings sah das in der Perspektive der Zeitgenossen anders aus. Wenngleich positive Kommentare nicht fehlten, so herrschte Unsicherheit vor, ob diese Tendenz dauerhaft sein würde ${ }^{94}$.

Nachdem die Bundesrepublik im November 1951 ihre kumulative Schuldnerposition in der EZU abgebaut hatte, nahm sie zum 1.Januar 1952 die Liberalisierung mit 57 Prozent wieder auf ${ }^{95}$. Dieser Prozentsatz war im Zentralbankrat umstritten gewesen. Da einige Mitglieder des Zentralbanksystems „einen ziemlich erheblichen Schock " erwarteten, hatten sie für einen niedrigeren Satz plädiert ${ }^{96}$. Wie Tabelle 6 zeigt, blieb dieser Schock jedoch aus: Zwar wuchsen die Importe aus dem EZU-Raum im ersten Quartal 1952 um 14 Prozent, doch blieb noch immer ein Überschuß bestehen. Das änderte sich auch in den folgenden Monaten nicht - selbst wenn der Zentralbankrat immer wieder Exporteinbrüche aufgrund der europäischen Entliberalisierungsmaßnahmen sowie einen Anstieg der deutschen Importe aufgrund der Erhöhung der deutschen Liberalisierungsquote befürchtete ${ }^{97}$.

Da die Überschüsse zum EZU-Raum anhielten, erreichte die Bundesrepublik im März 1952 die erste Tranche als Gläubiger, und im Sommer 1952 hatte sie ihre EZU-Quote fast erschöpft. Das führte dazu, daß sich zeitweise in Bonn und in Frankfurt sogar eine gewisse „Nervosität“ ausbreitete. Im Mai 1952 stellte Treue fest, die deutsche Zahlungsbilanz zur EZU verbessere sich „in beängstigendem Rhythmus“. Im Juni bezeichnete Dr. Gustav Gerbaulet, Leiter der Ab-

93 BBK, B 330/51, 6c (Devisenbewirtschaftung), Vermerk, 31.5.1952.

94 Die deutsche Zahlungsposition zur EZU wurde im Geschäftsbericht der BdL als ,insgesamt gefestigter als jemals zuvor" bezeichnet. Vgl. BdL, Geschäftsbericht 1951, S. 60.

$95 \mathrm{Vgl}$. BAnz Nr. 4, 8.1.1952.

96 BBK, B 330/51, ZBR, Stenogramm, 19.12.1951.

97 Vgl. BBK, B 330/52, ZBR, Protokoll, 7.2. 1952. Zum 1.4.1952 wurde die deutsche Liberalisierung auf $77 \%$, zum 1.8.1952 auf 80,9\% erhöht. Vgl. BAnz Nr.69, 1.4.1952 und BAnz Nr.154, 12.8.1952. 
Tabelle 6: Entwicklung des Im- und Exportes und der Reserven 1951/52

\begin{tabular}{lccccccc} 
& \multicolumn{2}{c}{ OEEC-Raum } & \multicolumn{2}{c}{ Off-Set-Raum } & \multicolumn{2}{c}{ Dollar-Raum } & Reserven \\
& $\begin{array}{l}\text { Export } \\
\text { Mio. \$ }\end{array}$ & $\begin{array}{c}\text { Import } \\
\text { Mio. \$ }\end{array}$ & $\begin{array}{c}\text { Export } \\
\text { Mio.\$ }\end{array}$ & $\begin{array}{c}\text { Import } \\
\text { Mio. \$ }\end{array}$ & $\begin{array}{c}\text { Export } \\
\text { Mio. \$ }\end{array}$ & $\begin{array}{c}\text { Import } \\
\text { Mio. \$ }\end{array}$ & $\begin{array}{c}\text { Gold/\$ } \\
\text { Mio. \$ }\end{array}$ \\
\hline I-51 & 542,8 & 599,6 & $\mathbf{9 8 , 2}$ & 81,4 & 70,6 & 128,3 & 182,3 \\
II-51 & $\mathbf{6 2 4 , 9}$ & 393,6 & 131,0 & 110,9 & 87,9 & 169,0 & 295,4 \\
III-51 & 715,7 & 544,5 & 148,7 & 124,6 & 96,0 & 172,7 & 431,5 \\
IV-51 & 732,1 & 501,3 & 145,3 & 116,6 & 79,7 & 169,8 & 365,3 \\
I-52 & 729,2 & 569,3 & 141,3 & 139,1 & 75,7 & 196,8 & 224,4 \\
II-52 & 721,6 & 512,4 & 169,7 & 121,8 & 85,2 & 135,7 & 447,1 \\
III-52 & 751,0 & 554,7 & 168,3 & 111,3 & 97,7 & 143,0 & 541,3 \\
IV-52 & 803,5 & 782,9 & 164,8 & 108,1 & 125,9 & 128,4 & 636,7 \\
\hline
\end{tabular}

Quelle: Statistisches Handbuch der BdL, S.256ff.

teilung III des Marshallplan-Ministeriums (Zwischenstaatliche Wirtschaftsbeziehungen), die Überschüsse als „galoppierende Fettsucht ${ }^{\alpha 98}$. Wenn auch die Zahlungsbilanzsituation zum EZU-Raum befriedigend war, so herrschte weiterhin eine gewisse Unsicherheit vor. Beispielsweise zweifelte Emminger, ob die Überschüsse anhalten würden ${ }^{99}$. Im August 1952 rechnete der Zentralbankrat für den Herbst mit einem Anstieg der Einfuhren. Im November sorgte sich Pfleiderer erneut um die Zahlungsbilanz ${ }^{100}$.

Im Vergleich zum EZU-Raum war die Situation zum Dollarraum um einiges prekärer. Bei zurückgehendem Export und ansteigendem Import klaffte die Dollarlücke im Winter 1951/52 wieder auf. Vocke wandte sich sogar wegen des Reservenschwundes an Adenauer und Schäffer ${ }^{101}$, während Anfang 1952 die BdL erneut eine restriktivere Haltung bei der Devisenzuteilung einnahm ${ }^{102}$. Erst ab Sommer 1952 verbesserte sich die Lage bei steigenden Exporten und sinkenden Importen, so daß im vierten Quartal 1952 die Zahlungsbilanz zum Dollarraum - Bewirtschaftungsmaßnahmen vorausgesetzt - fast ausgeglichen war. Diese Entwicklung erlaubte, die aus den EZU-Überschüssen resultierenden Goldzahlungen nicht mehr nur für Dollarimporte, sondern zum Teil auch für eine Reserven-

98 BAK, B 146/565, Sachs, Vermerk, 21.5.1952, Gerbaulet an Born, 21.6.1952; BAK, B 146/527, Treue an Gerbaulet, 5.8.1952. Bei einer Gelegenheit spricht Gerbaulet sogar von „Verzweiflung“ . Vgl. BAK, B 146/527, Gerbaulet an Treue, 17.9.1952.

99 Vgl. BBK, B 330/1310, Emminger an Möller, 19.7.1952; BAK, B 102/56893, Gurski an BMM, 18.8.1952.

100 Vgl. BBK, B 330/59, ZBR, Protokoll, 13./14.8.1952; BBK, B 330/62, ZBR, Stenogramm, $12 . /$ 13.11.1952; BBK, B 330/63, ZBR, Stenogramm, 26.11.1952.

101 Vgl. BBK, B 330/2011, Vocke an Adenauer, 14.11.1951; BBK, B 330/2043, Vocke an Schäffer, 7.1.1952.

${ }^{102}$ Vgl. BAK, B 102/12582.2, BMM, BMWi, BML, Entwurf einer Kabinettsvorlage über das Programm der Einfuhren aus dem Dollarraum, 29.1.1952; BAK, B 102/13720.2, I A 5 Schmitt, Vermerk betreffs EZU-Zahlungsposition der Bundesrepublik Deutschland, 12.3.1952. 
aufstockung zu verwenden. Trotz dieser Aufstockung waren die Reserven laut Vocke noch immer „unzureichend“ ${ }^{103}$. Ferner war Emminger überzeugt, daß die positive Entwicklung zum Dollarraum nur auf besondere Faktoren zurückzuführen sei. In „absehbarer Zeit“ würde die Zahlungsbilanz zum Dollarraum wieder ein Defizit aufweisen. Das Grundproblem, die Dollarlücke, sei weit von einer Lösung entfernt ${ }^{104}$.

Insgesamt führte die Zahlungsbilanzsituation zu folgender Konstellation: Zum einen sah das Zentralbanksystem eine Konvertibilität gegenüber dem Dollarraum einhellig als nicht realisierbar an. Zum anderen gab es ein Interesse an der Konvertibilität, vor allem an der Konvertibilität der anderen Währungen. Dadurch würden - so stellte die BdL auch in ihren Veröffentlichungen heraus - die Auswirkungen der „gegenwärtigen unterschiedlichen Zahlungsbilanzlage“ erheblich gemildert werden ${ }^{105}$.

Verteidigungslasten

Außer der unsicheren Zahlungsbilanzentwicklung waren auch die Verteidigungskosten ein Gesichtspunkt, der immer in Zusammenhang mit dem Abbau der Devisenbewirtschaftung gesehen wurde. Angesichts des Kalten Krieges war schon Ende der vierziger Jahre absehbar, daß die Bundesrepublik zur Verteidigung des westlichen Blockes beitragen sollte. Mit dem Vorschlag des französischen Ministerpräsidenten René Pleven, eine Europäische Verteidigungsgemeinschaft (EVG) zu errichten, und mit den im Februar 1951 beginnenden Verhandlungen konkretisierten sich die Pläne. Wenngleich die deutsche Zentralbank keine Kredite zur laufenden Haushaltsfinanzierung geben durfte und die Verteidigungskosten somit aus den laufenden Einnahmen des Bundes finanziert werden mußten, war nur allzu offensichtlich, daß die deutsche Wiederaufrüstung bzw. die Zusammenlegung der europäischen Verteidigungskräfte auch monetäre Auswirkungen haben würde.

Die Diskussionen über die deutsche Wiederaufrüstung führten zu zahlreichen Schätzungen und Meinungen zur vertretbaren Höhe eines bundesdeutschen Verteidigungsbeitrages. Wie Lutz Köllner und Hans-Erich Volkmann hervorheben, zeichneten sich alle Stellungnahmen durch einen mahnenden und zurückhaltenden Grundtenor aus ${ }^{106}$. Auch die BdL reihte sich hier ein. Vocke lief gegen die von den USA vorgebrachten Verteidigungskosten in Höhe von $13 \mathrm{Mrd}$. DM Sturm und sah sie als „Proklamation der Inflation“ an. Selbst im Herbst 1954

103 BBK, B 330/2043, Vocke an Schäffer, 4.8.1952.

104 BAK, B 102/13720.1, Emminger an Schmitt, 23.9.1952 und BBK, NL Emminger, Dollarlücke, Emminger, Dollarproblem, 1.12.1952. Im Marshallplan-Ministerium wurde die Meinung der BdL geteilt. Vgl. BAK, B 146/527, BMM an BMWi, 24.7.1952. Die konvertiblen Reserven deckten im vierten Quartal 1952 zwei Monatseinfuhren nicht ab.

105 BBK, NL Emminger, Konvertibilität/Abbau der Devisenzwangswirtschaft I 1949-52, Emminger, Abbau der Devisen-Zwangswirtschaft, 15.5.1952. Vgl. BdL, Geschäftsbericht 1951, S.1.

106 Köllner/Volkmann, Aspekte, S.759ff., hier S. 804. 
warnte Emminger noch vor direkten wie indirekten Auswirkungen der Aufrüstung ${ }^{107}$. Trotz dieser Warnungen blieb die BdL in bezug auf die EVG-Verhandlungen gleichmütig und griff kaum ein. Das lag vor allem daran, daß sich die sechs zukünftigen EVG-Mitglieder früh darauf einigten, die Beiträge weitgehend in nationaler Währung zu entrichten. Darüber hinaus konnten sich die Verhandlungspartner auf eine Transferschutzklausel von 15 Prozent verständigen: Schließlich sorgten sich alle zukünftigen EVG-Mitglieder um die Auswirkungen der Verteidigungsgemeinschaft auf die Währungsreserven, so daß alle Beteiligten übermäßige Transfermöglichkeiten vermeiden wollten ${ }^{108}$. Mit dieser 15-ProzentKlausel war für die BdL „eine akzeptable Lösung“ gefunden. Im folgenden ängstigte sie sich nicht mehr um den Transfer. Ganz im Gegenteil: Eine Erhöhung dieses Satzes lehnte sie ab, da das einer Vergrößerung der "Devisen-Chancen“ gleichkäme. Diese wollte die BdL vermeiden, denn höhere „Devisen-Chancen“ würden die Bundesrepublik nur in die "unangenehme Lage einer permanenten Gläubigerposition in der EZU“ bringen ${ }^{109}$.

Obwohl also die Verteidigungskosten bei verschiedenen Gelegenheiten als Hindernis auf dem Weg zur Konvertibilität angeführt wurden, spielten sie dann im Zusammenhang mit der EVG keine wesentliche Rolle. Wie Bernard auch gegenüber dem Vorsitzenden des Bundestagsausschusses für Wirtschaftspolitik, Wilhelm Naegel, ausführte, seien im Vertrag für die währungspolitische Seite ausreichende Schutzklauseln an mehreren Stellen enthal$\operatorname{ten}^{110}$.

Schulden- und Transferprobleme

Ein drittes Problem, das die BdL in den Jahren 1951/52 mit der Konvertibilität in Zusammenhang brachte, waren die deutschen Altschulden. Nach der Schuldenerklärung der Bundesregierung vom 6. März 1951 wurden im Sommer Verhandlungen über die Regelung der öffentlichen wie privaten deutschen Vor- und Nachkriegsschulden aufgenommen. Sie fanden mit der Unterzeichnung des Londoner Schuldenabkommens im Februar 1953 ihren Abschluß $\Re^{111}$.

Auch wenn die BdL an der Regelung der Altschulden interessiert war ${ }^{112}$, verfolgte sie die entsprechenden Verhandlungen argwöhnisch und begutachtete die daraus resultierenden Devisenbelastungen aufmerksam. Sie schätzte die deutsche

107 BBK, B 330/2043, Vocke an Schäffer, 7.1.1952; BBK, B 330/3380, Emminger, Mögliche Auswirkungen der Aufrüstung auf die deutsche Zahlungsbilanz, 29.10.1954. Wolf bezeichnete die Ausführungen Emmingers als übertrieben. Vgl. BBK, B 330/3380, Wolf, Stellungnahme zu dem Vermerk von Herrn Dr. Emminger, 5.11.1954.

108 Vgl. Köllner/Volkmann, Aspekte, S.858ff.; Finanzprotokoll des EVG-Vertrages in: BGBL 1954 II, S. $403 \mathrm{f}$.

109 BBK, B 330/3478, Emminger, Transferprobleme im Rahmen der Verteidigungsgemeinschaft, 19.1.1952.

110 Vgl. BBK, B 330/3317, Bernard an Naegel, 4.11.1952.

111 Abgedruckt in: BGBL 1953 II, S. $331 \mathrm{ff}$.

112 Vgl. BBK, B 330/51, ZBR, Stenogramm, 19.12.1951: Vgl. hierzu auch S. $111 \mathrm{f}$. 
Transferfähigkeit gering ein ${ }^{113}$. Vocke hielt sogar die letztendlich vereinbarten jährlichen Zahlungen in Höhe von 567 Mio. DM für untragbar ${ }^{114}$. Durch solche Belastungen würde - so stellte er gegenüber Hermann J.Abs, Leiter der deutschen Delegation bei den Schuldenverhandlungen, fest - die Konvertibilität auf Jahre hinaus unmöglich gemacht ${ }^{115}$. Der Replik von Abs, daß das Londoner Schuldenabkommen eine Voraussetzung für die Konvertibilität sei, da es Kapitalimporte ermöglichen würde, pflichtete die BdL nicht bei. Für sie standen die Belastungen im Vordergrund, denn sie betrachtete die damit eröffnete Möglichkeit, die Konvertibilität durch Kapitalimporte abzusichern, mit Skepsis ${ }^{116}$.

Wenn auch die Belastungen des Londoner Schuldenabkommens laut BdL die Erreichung der Konvertibilität vorerst ausschlossen, so machten sie die Konvertibilität andererseits notwendig. In seiner Sitzung am 31.Juli 1952 stellte der Zentralbankrat heraus, daß zur Erfüllung der deutschen Verpflichtungen „auch eine konforme Handelspolitik der Gläubigerländer vonnöten ist “117. Die Bundesrepublik müsse Überschüsse erwirtschaften und diese konvertieren können. Vocke wollte deshalb sogar eine entsprechende, verbindliche Verpflichtung der deutschen Gläubiger in das Schuldenabkommen verankert wissen. Die deutschen Gläubiger sollten seines Erachtens verpflichtet werden, durch eine liberale Handelspolitik und durch Gewährung der Konvertibilität deutsche Zahlungsbilanzüberschüsse und damit die entsprechenden Transfers zu ermöglichen ${ }^{118}$.

Als die BdL im Verlaufe des Jahres 1951/52 in der Diskussion über die Konvertibilität Position beziehen mußte, vertrat sie eine vorwiegend ablehnende $\mathrm{Hal}$ tung. Ihre oftmals verlautbarte Befürwortung der Konvertibilität kann deshalb als ein Lippenbekenntnis bezeichnet werden. Sobald es konkret wurde, votierte die Bank für den Status quo. Zum Teil verlangte sie sogar eine sicherere Verankerung des regionalen Prinzips und befürwortete in diesem Zusammenhang eine supranationale EZU. Die Fortführung der OEEC-Liberalisierung, feste Wechselkurse und Regionalismus waren ihr wichtiger als Konvertibilität und Abbau der Devisenbewirtschaftung. Gleichwohl kann die BdL nicht als eine prinzipielle Konvertibilitätsgegnerin bezeichnet werden. Wenngleich sie auch anläßlich der belgischen Gläubigerkrise den Goldstandard kritisierte, war ihre Haltung zu einem großen Teil durch die zu dieser Zeit vorherrschenden Umstände bedingt. Tatsächlich glaubte die BdL, daß eine Erklärung der Konvertibilität wegen der unsicheren Zahlungsbilanzaussichten und den anstehenden Belastungen aus der

113 Vgl. BBK, B 330/2012, BdL, Die deutsche Transferfähigkeit, 2.2.1952. Deshalb wollte sie das Schuldenproblem auch vertagen. Vgl. BAK, NL Hallstein/122, Hallstein, Notizen zur Sitzung über Finanzprobleme, 14.5.1952.

$114 \mathrm{Vgl}$. Abs, Wiederherstellung, S. 34 .

115 Vgl. ebenda, S. 36 .

116 Siehe hierzu S. $110 \mathrm{ff}$.

117 BBK, B 330/58, ZBR, Protokoll, 31.7.1952.

118 Vgl. BBK, B 330/3379, Vocke, Vermerk, 5.8.1952. Vgl. auch BBK, B 330/61, ZBR, Protokoll, 15.10. 1952. Das Abkommen enthielt nur die von Vocke angefeindete, unverbindlichere Formulierung „erleichtert werden würden“. Vgl. BGBL 1953 II, S. 481. 
deutschen Verschuldung vorerst unmöglich sei. Immer wieder betonte sie, die Konvertibilitätsfrage sei „eine Frage des Könnens, nicht des Wollens“. Die Erreichung der Konvertibilität sei aber "selbstverständlich" anzustreben ${ }^{119}$. Ob und inwieweit sie dieses Ziel in den nächsten Jahren, die weitere deutsche Überschüsse und somit eine Vergrößerung des Handlungsspielraums sahen, tatsächlich intensiv verfolgte, wird im folgenden untersucht.

\section{Gründe für und gegen die Konvertibilität}

In der Ablehnung der belgischen Forderungen und der Erhardschen Ideen zeigt sich eine provisorische, erste Reaktion der BdL. Dabei hatte sie vor allem die ungenügenden deutschen Währungsreserven und die noch unsicher erscheinende deutsche Zahlungsbilanzsituation vor Augen gehabt. In den folgenden Jahren mußte die Bank jedoch ihre Position überdenken. Vor allem mußte sie ihrem vergrößerten Handlungsspielraum und den mit den deutschen Überschüssen wachsenden Kosten des Regionalismus wie der Inkonvertibilität ihrer Kredite Rechnung tragen.

\section{Exportmöglichkeiten und europäische Zablungsbilanzentwicklungen}

Nach den Devisenkrisen des Winters 1950/51 hatte die Bundesrepublik im Jahre 1952 beträchtliche Überschüsse zum EZU-Raum erwirtschaftet. Gleichzeitig hatte sich das Defizit zum Dollarraum - Bewirtschaftung vorausgesetzt - beträchtlich verringert. Wie Tabelle 7 (S.144) zeigt, setzte sich diese Entwicklung auch im Jahre 1953 fort. Selbst wenn man den währungspolitischen Akteuren eine gewisse Zeit für die "geistige Bewältigung des plötzlichen Umschwunges" ${ }^{\text {“120 }}$ zugesteht, war seit 1953 nicht mehr zu leugnen, daß die deutsche Zahlungsbilanzentwicklung stabil war. Außerdem waren die deutschen Währungsreserven stetig alimentiert worden, und sie bildeten inzwischen ein ansehnliches Polster.

Tatsächlich sah die BdL seit 1953 die Reserven nicht mehr als ein Problem an. Darüber hinaus war sie nicht mehr permanent von der Möglichkeit eines Zahlungsbilanzumschwunges geplagt, so daß sie die DM sogar als eine starke Währung einschätzte ${ }^{121}$. Damit war der deutsche Handlungsspielraum vergrößert.

119 BBK, B 330/57, 6c (Devisenbewirtschaftung), Vermerk, 31.5. 1952; BBK, NL Emminger, Konvertibilität/Abbau der Devisenzwangswirtschaft I 1949-52, Emminger, Abbau der Devisen-Zwangswirtschaft, 15.5.1952. Deutlich wird diese zwiespältige Haltung der BdL anläßlich der Vorlage eines OEEC-Memorandums zur Konvertibilität. Vgl. HAEC, OEEC-TRA 333, MBC (52)65, 1. Rev. vom 1.12.1952. Am 11.11.1952 bedauerte Vocke die ,ausgesprochen negative Haltung (dieses Dokuments, M.D.) zur Frage der Konvertibilität ${ }^{*}$. Am 12.11.1952 offenbarte er in gleicher Angelegenheit seine eigene negative Haltung zur Konvertibilität. Vgl. BAK, B 146/480, Vocke an Blücher, 11.11.1952; Albrecht, Vermerk, 12.11.1952.

120 Emminger stellt in seinen Memoiren die Schwierigkeiten dieser "geistigen Bewältigung “ heraus. Vgl. Emminger, D-Mark, S.68ff.

121 Vgl. BBK, B 330/2012, Vocke an Erhard, 11.12.1952. 
Tabelle 7: Handels- und Dienstleistungsbilanz, Reserven 1952-1954

EZU-Raum Dollar-Raum Off-Set-Raum Insgesamt Reserven

Gold/\$

\begin{tabular}{lrrrrr} 
& Mio.\$ & Mio.\$ & Mio.\$ & Mio.\$ & Mio.\$ \\
\hline I-52 & 110,7 & $-107,5$ & $-3,3$ & $-0,1$ & 334,4 \\
II-52 & 181,5 & $-2,2$ & 42,3 & 221,6 & 447,1 \\
III-52 & 163,4 & 12,7 & 53,6 & 229,7 & 541,3 \\
IV-52 & 5,5 & 68,9 & 52,2 & 115,6 & 636,7 \\
I-53 & 51,2 & 63,7 & 18,7 & 113,6 & 718,9 \\
II-53 & 148,4 & 51,2 & 46,6 & 246,2 & 829,2 \\
III-53 & 123,3 & 77,4 & 44,3 & 245,0 & 992,5 \\
IV-53 & 211,2 & 91,7 & 43,2 & 346,1 & 1169,2 \\
I-54 & 182,3 & 77,2 & 3,2 & 262,7 & 1340,3 \\
II-54 & 156,0 & 43,5 & 3,1 & 202,6 & 1449,1 \\
III-54 & 165,2 & 111,7 & 2,2 & 279,1 & 1779,3 \\
IV-54 & 160,9 & 71,0 & $-19,3$ & 212,6 & 1923,5 \\
\hline
\end{tabular}

Quelle: Statistisches Handbuch der BdL, S.256ff.

$\mathrm{Da}$ die $\mathrm{BdL}$ bisher stets die unsichere deutsche Zahlungsbilanzsituation angeführt hatte, gab es nun einen Grund zur Revision ihrer konvertibilitätsskeptischen Haltung. Weil die BdL jetzt aber keineswegs nur die deutsche Situation, sondern vielmehr auch die europäische Zahlungsbilanz- und Reservenentwicklung als ausschlaggebend ansah, korrigierte sie ihre Haltung nicht, denn die europäische Situation war weitaus weniger positiv. Zwar wuchsen die konvertiblen europäischen Reserven in den Jahren 1952 bis 1954 insgesamt an ${ }^{122}$, doch sind in diesen aggregierten Zahlen nicht nur die positive deutsche Entwicklung, sondern auch die ebenfalls positiven Entwicklungen Belgiens, der Niederlande und der Schweiz enthalten, so daß auf den ersten Blick die ungenügenden Reserven einer Vielzahl von Ländern verdeckt bleiben. Der Anstieg der konvertiblen europäischen Währungsreserven spiegelt die gesamteuropäische Situation nur ungenügend wider ${ }^{123}$ : Die britische Situation war weiterhin prekär, denn die Reserven des Vereinigten Königreiches waren im Verlauf des Jahres 1951 er-

${ }^{122}$ Die europäischen konvertiblen Reserven beliefen sich Ende 1952 auf 8,9 Mrd. \$, wuchsen bis Ende 1953 auf 11,2 Mrd. \$ an und erreichten Ende 1954 den Stand von 13,0 Mrd. \$. Vgl. OEEC, 7 th Annual Report (1956), S. 104.

${ }^{123}$ Das ist ein Grund, warum die von Eichengreen vorgelegte Analyse der Währungsreserven - vgl. Eichengreen, Reconstructing, S. $53 \mathrm{ff}$. - zu kritisieren ist. Ferner ist festzuhalten, daß Eichengreen nicht zwischen konvertiblen und nicht-konvertiblen Währungsreserven unterscheidet. Seine Quelle - die International Financial Statistics des IWF - subsumieren EZU-Guthaben oftmals unter die Währungsreserven. Des weiteren sollten die Reserven nicht dem Export, sondern dem Import gegenübergestellt werden, wobei zu bedenken ist, daß der Import administrativ beschränkt wurde und nicht mit den Importbedürfnissen gleichzusetzen ist. Insgesamt kann deshalb der Meinung Eichengreens, die europäischen Währungsreserven wären ausreichend gewesen, so nicht zugestimmt werden. 
heblich gesunken, und die Liberalisierung mußte eingeschränkt werden ${ }^{124}$. Auch Frankreich litt unter Zahlungsbilanzproblemen, so daß es seine Liberalisierung Anfang 1952 suspendieren mußte ${ }^{125}$. Gleichfalls wenig zufriedenstellend waren die norwegischen, dänischen und irischen Reservenpositionen. Schließlich akkumulierte Italien seit 1953/54 erhebliche Defizite zum EZU-Raum, was sogar seine OEEC-Liberalisierung in Frage stellte ${ }^{126}$. Wegen dieser europäischen Reservensituation stand die BdL trotz der gefestigten deutschen Situation und trotz ausreichender deutscher Reserven weiterhin der Konvertibilität skeptisch gegenüber.

Die Motivation, die hinter dieser Zurückhaltung stand, war, daß laut BdL das deutsche Interesse nicht in erster Linie in der Konvertibilität der DM, sondern vielmehr in der Konvertibilität der anderen europäischen Währungen lag. Im Gegensatz zum Vereinigten Königreich, das die Konvertibilität der eigenen Währung für Ausländer anstrebte, sah die BdL die Konvertibilität der DM für Ausländer nicht als ein wichtiges Ziel an. Im Vordergrund stand für sie die Konvertibilität der anderen Währungen in Form der weiteren Liberalisierung der OEECMitgliedsländer. Der Grund für dieses spezifische deutsche Interesse lag darin, daß die Konvertibilität der anderen nach innen den deutschen Export sichern würde, denn im Jahre 1953 wurden über 70 Prozent der deutschen Exporte im EZU-Raum abgesetzt ${ }^{127}$. Ferner war der BdL die Konvertibilität der anderen Währungen nach außen wichtig, wenn auch im Vergleich zur Liberalisierung zweitrangig. Diese Konvertibilität würde nämlich erlauben, das traditionelle deutsche Defizit mit dem Dollarraum über die Exportüberschüsse mit anderen Ländern auszugleichen ${ }^{128}$. Die Konvertibilität der DM hingegen befürwortete die Bank in erster Linie nur als Inländer-Konvertibilität, also als eine schrittweise Lockerung der Devisenrestriktionen. Eine solche Konvertibilität würde den Rohstoffbezug verbilligen und dadurch die Wettbewerbsfähigkeit der deutschen Wirtschaft stärken. Darüber hinaus wäre eine solche Politik eine Good Creditor Policy, denn sie würde die Stellung der europäischen Defizitländer erleichtern und ihnen erlauben, die OEEC-Liberalisierung aufrechtzuerhalten, was wiederum den deutschen Export sichere ${ }^{129}$. Eine Konvertibilität der DM für Ausländer wurde nicht als erstrebenswert angesehen. Hier hielt Emminger fest: „An der

${ }^{124}$ Die konvertiblen Reserven des Vereinigten Königreiches sanken von 3300 Mio. \$(1950) auf 1846 Mio. \$ (1952) und erholten sich danach nur zögernd. Vgl. International Financial Statistics, Vol. XIII, No. 6, June 1960, S. 258.

125 Die konvertiblen Reserven Frankreichs sanken von 791 Mio. \$ (1950) auf 616 Mio. \$ (1951). Sie erholten sich nur aufgrund erheblicher amerikanischer Militärhilfe. Vgl. International Financial Statistics, Vol. XIII, No. 6, June 1960, S. 124; Bossuat, L'aide, S. $795 \mathrm{ff}$.

126 Vgl. Fauri, Negotiating, S. $68 \mathrm{ff}$.

${ }^{127}$ Vgl. Statistisches Jahrbuch der BRD 1954, S. 323 f.

${ }^{128}$ Vgl. BBK, NL Emminger, Konvertibilität/Abbau der Devisenzwangswirtschaft II 1953, o. V. (vermutlich Emminger), Konvertierbarkeit der Währungen von Deutschland her gesehen, o.D. (Mitte 1953)

129 Ebenda. 
Konvertierbarkeit der D-Mark für Ausländer ist Deutschland im Gegensatz zu England weniger interessiert, da es nicht den Ehrgeiz haben kann, die D-Mark zur Welthandelswährung oder Frankfurt oder Hamburg zu einem internationalen Finanzzentrum zu machen. Außerdem besteht im deutschen Falle wenig Hoffnung [.. .], daß die Ausländer die DM-Beträge zum Teil als Reserve behalten würden, statt sie in Dollar umzutauschen. " 130

Als Konsequenz forderte die BdL immer wieder, die Situation der europäischen Schuldnerländer zu beachten. Sie betonte, alle europäischen Länder auch die schwächeren - müßten in einen Konvertibilitätsschritt einbezogen werden. Gemeinsame Fortschritte seien notwendig. Die Bundesrepublik könne keinesfalls alleine vorgehen ${ }^{131}$. Die deutsche Zahlungsbilanzsituation und Reservenentwicklung war für die BdL nicht der entscheidende Parameter für eine Annäherung an die Konvertibilität. Interessiert an der Sicherung des deutschen Exports, war die Position der anderen Länder maßgebend.

\section{Kapitalmangel, Kapitalimport und Liberalisierung der Erträgnisse}

In der einschlägigen Literatur herrscht Einigkeit, daß die Bundesrepublik bis zur Mitte der fünfziger Jahre unter Kapitalmangel litt ${ }^{132}$. Das hob auch die Bank in ihren Geschäftsberichten immer wieder hervor. Trotz verschiedener Förderungsmaßnahmen - wie zum Beispiel dem Ersten Gesetz zur Förderung des Kapitalmarktes vom 15. Dezember 1952 - lag der Kapitalmarkt „danieder“, war „zu eng" und konnte die notwendigen Mittel für die anhaltend hohe Investitionstätigkeit nicht zur Verfügung stellen. Vor allem kam - so klagte die BdL - die Wirtschaft auf dem Kapitalmarkt nicht zum Zuge ${ }^{133}$. Wenn sich auch, wie Abbildung 4 zeigt, der Wertpapierabsatz seit $1949 \mathrm{mehr}$ als verdreifacht hatte, so wird gleichzeitig deutlich, daß der Großteil dieses Absatzes den öffentlichen Händen und Pfandbriefanstalten zugute kam. Der Anteil der Aktien und Industrieobligationen war noch immer verhältnismäßig gering. Er belief sich zusammen auf 17 bzw. 21 Prozent (1949 bzw. 1953).

Diese Situation brachte auch immer wieder die Frage nach Kapitalimporten auf. Wie oben gezeigt, sah die BdL von Anfang an Auslandskredite als unabdingbar für den westdeutschen Wiederaufbau an. Da sie aber gleichzeitig Zweifel hinsichtlich der internationalen Kapitalverflechtung und der möglichen Devisenbe-

130 Ebenda.

131 Vgl. BBK, B 330/1313, Emminger, Gutachten über die Weiterentwicklung der EZU von Mr. Harrod, 12.8.1952; BBK, B 330/2012, Vocke an Erhard, 19.3.1953; BBK, B 330/2056, Direktorium, Protokoll, 15.5.1953; BBK, B 330/3379, Vocke, Zur Frage der Konvertibilität, 9.12.1953.

132 Vgl. Giersch/Paqué/Schmieding, Miracle, S.45f.; Röhl, Entwicklung; Roskamp, Capital Formation, $\mathrm{S} .53 \mathrm{ff}$.

133 BdL, Geschäftsbericht 1952, S.15ff;; dies., Geschäftsbericht 1953, S. $19 \mathrm{ff.} \mathrm{Das} \mathrm{Erste} \mathrm{Gesetz} \mathrm{zur}$ Förderung des Kapitalverkehrs ist abgedruckt in BGBL 1952 I, S. $793 \mathrm{ff}$. Es fand keine ungeteilte Zustimmung. Die BdL monierte, daß durch die Steuerbefreiung für Zinsen von festverzinslichen Wertpapieren die "Zinswahrheit" nicht zum Zuge käme. 
Abbildung 4: Wertpapierabsatz 1949-1953

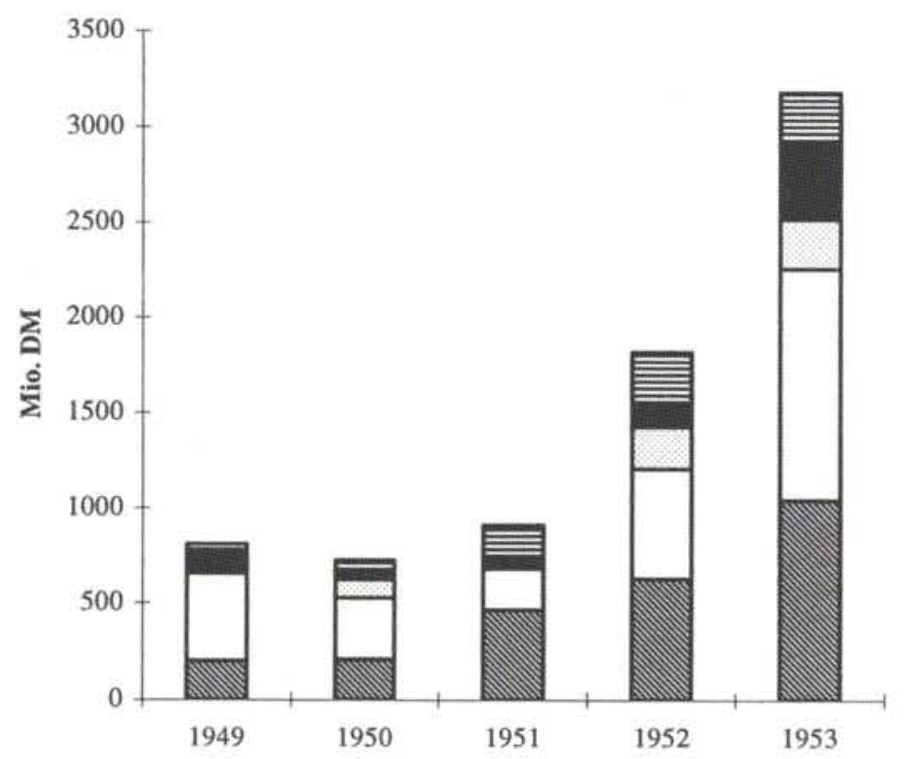

日aktien

폴. Ind. Oblig.

口Schuldverschr. 口Öff. Hănde

\$Pfandbriefe

Quelle: BdL, Geschäftsbericht 1953, S. 53.

lastung hegte, richtete sie ihr Augenmerk in erster Linie auf einen Weltbankkredit $^{134}$. Dieses Projekt zeigte jedoch schon bald seine problematischen Seiten. Bereits im Herbst 1952 befürchtete Vocke, daß an einen Weltbankkredit untragbare Bedingungen - wie zum Beispiel die Freigabe der deutschen Subskriptionsquote für einen Kredit an Jugoslawien - geknüpft würden ${ }^{135}$. Als es im weiteren Verlauf auch zu Unstimmigkeiten über den Anleihebetrag, die Fristigkeit und die Bedingungen kam, wurde das Projekt einer Weltbankanleihe im Herbst 1953 endgültig ad acta gelegt ${ }^{136}$. Mit diesem Schritt lag es nahe, sich erneut anderen ausländischen Kapitalquellen zuzuwenden. Die Funktion der Konvertibilität, Kapitalimporte zu ermöglichen, wurde deshalb wieder bedeutend ${ }^{137}$.

134 Vgl. S. 113.

135 Vgl. BBK, B 330/2021, Vocke an Erhard, 2.10.1952; Kabinettsprotokolle V (1952), 256. Kabinettssitzung am 7.11.1952, S.665.

136 Vgl. BBK, B 330/3379, Vocke, Aufzeichnung über mein Gespräch mit Mr. Black, 7.6.1953; BBK, B 330/2021, Vocke an Erhard, 9.7.1953 und 21.8.1953; BBK, B 330/2056, Direktorium, Protokoll, 30.7.1953; BBK, B 330/70, ZBR, Protokoll, 8.7.1953; BBK, B 330/73, ZBR, Protokoll, 28.10.1953 und 25.11.1953; Kabinettsprotokolle VI (1953), 8. Kabinettssitzung am 17.11.1953, S. $513 \mathrm{f}$.

${ }^{137}$ Giersch/Paqué/Schmieding, Miracle, S.115, heben diese Funktion der Konvertibilität hervor. Sie vermuten, daß die Konvertibilität zu Kapitalzuflüssen geführt und dadurch die Rekonstruktion beschleunigt hätte. Eichengreen, Reconstructing, S.110ff., hat diese Vermutung mit cliometrischen Methoden untersucht und zurückgewiesen. 
Die Haltung der BdL zu Kapitalimporten von privaten, ausländischen Gläubigern war aber weiterhin zwiespältig. Einerseits sprach sich das Direktorium für eine größere Elastizität bei der Frage amerikanischer Anleihen aus, wobei sich Vocke gegen den von Überfremdungsängsten geplagten Vorsitzenden des Bundesverbandes der Deutschen Industrie, Fritz Berg, wandte ${ }^{138}$. Andererseits aber blieben Vorbehalte bestehen. Die Bank verstand Kapitalimporte nicht als einen Ausgleichsposten in der Zahlungsbilanz. Ferner zweifelte sie, ob Auslandskredite überhaupt gegeben werden würden. Auch befürchtete sie, durch Auslandsverschuldung verwundbarer zu werden. Darüber hinaus sah sie Schwierigkeiten bei der Zurückzahlung der Kredite. In einer Zentralbankratsitzung bemerkte Pfleiderer zu Erhard: „Sie sagen, Amerika müßte uns dann eben Kredit geben. Was nützt aber eine Kreditgewährung, wenn Amerika gleichzeitig verbietet, daß Waren ins Land kommen? Die Kredite könnten ja niemals zurückgezahlt werden. ${ }^{" 139} \mathrm{Da}$ also die programmatischen Aussagen der Mitglieder des Zentralbanksystems widersprüchlich sind, kann nur die konkrete Politik der Zentralbank, z. B. bezüglich der Liberalisierung der Kapitalerträgnisse, Aufschlüsse über ihre Haltung geben. Selbst wenn die Diskussionen über die Liberalisierung der Kapitalerträgnisse in erster Linie den OEEC-Raum betrafen, können Hinweise gewonnen werden, ob und inwieweit die $\mathrm{BdL}$ an der Liberalisierung der Kapitalbewegungen und an der Konvertibilität interessiert war, um den Kapitalimport zu fördern.

Bei anhaltend hohen Überschüssen der Bundesrepublik zum EZU-Raum sprach die OEEC seit Sommer 1952 die Liberalisierung unsichtbarer Transaktionen und der vom Londoner Schuldenabkommen nicht geregelten Gebiete des Kapitalverkehrs an ${ }^{140}$. Im Dezember 1952 empfahl der OEEC-Rat, die deutsche Regierung möge Maßnahmen ergreifen, um die Liberalisierung der noch unter Vorbehalt stehenden unsichtbaren Transaktionen zu beschleunigen ${ }^{141}$. Diese Forderungen begegneten dem einmütigen deutschen Hinweis, daß vor Abschluß eines Schuldenabkommens keine Schritte unternommen werden könnten. Auch wurde hervorgehoben, daß die Zuständigkeiten der Bundesrepublik auf diesem Gebiet eingeschränkt seien ${ }^{142}$. Innerhalb der Bundesrepublik gab es aber einige Auseinandersetzungen über dieses Thema.

138 Vgl. BBK, B 330/2055, Direktorium, Protokoll, 8.5.1952; BBK, B 330/2014, Vocke an Berg, 12.12.1952.

139 BBK, B 330/57, ZBR, Stenogramm, 11.6. 1952. Macdonald stellte fest, "Vocke does not believe in dollar loans". Vgl. BoE, OV 34/38, Macdonald an Rootham, 4.2.1952.

140 Vgl. BAK, B 146/527, Germania Paris an BMM/BMWi, 24.7.1952. Vgl. auch BAK, B 102/6428.1, Werkmeister, Bericht an BMM, 2.8.1952. Das wichtigste vom Londoner Schuldenabkommen nicht erfaßte Gebiet war der Sperrmark-Komplex. Am 31.12.1953 beliefen sich die DM-Sperrguthaben auf 774,3 Mio. DM, von denen 234,6 Mio. DM auf den Dollarraum und 454,4 Mio. DM auf den EZU-Raum entfielen. Vgl. BdL, Geschäftsbericht 1953, S. 96.

141 Vgl. C (52) 339 Final vom 11.12. 1952, zit. in: BBK, B 330/3172, Behandlung laufender Zahlungen des Kapitalverkehrs in OEEC-Dokumenten, 2.1.1953.

142 Vgl. BBK, B 330/61, BMM, Memorandum der Bundesregierung über die Position Deutschlands in der EZU, 2.10.1952 (= MBC (52)63 vom 4.10.1952). Vgl. auch Deutsches Memorandum für 
Sowohl das Marshallplan-Ministerium als auch das Wirtschaftsministerium befürworteten die Liberalisierung der laufenden Kapitalerträgnisse. Für Erhard war der Kapitalimport ein Grund für seine Konvertibilitätskampagne, und schon im Sommer 1952 hatte Kurt Schöne, Leiter der Abteilung V C des Wirtschaftsministeriums (Zahlungsverkehr mit dem Ausland), bedauert, daß eine Nicht-Liberalisierung der Kapitalerträgnisse eine Neuaufnahme von Auslandskrediten "praktisch unterbinden " würde ${ }^{143}$. Im Wirtschaftsministerium wurde nämlich der Kapitalverkehr als Ausgleichsposten in der Zahlungsbilanz verstanden ${ }^{144}$. Im Gegensatz dazu verhielt sich die BdL zurückhaltend ${ }^{145}$. Bei den entsprechenden Ressortbesprechungen vertrat sie bis zum Sommer 1953 zusammen mit Abs und dem Finanzministerium eine strikt ablehnende Meinung. Abgesehen davon, daß laut Emminger Kapitalbewegungen nicht zum Ausgleich der Zahlungsbilanz herangezogen werden sollten ${ }^{146}$, war die BdL besorgt, daß „ein Rückfall [...] auf dem Kapitalsektor viel weittragender [wäre] als auf dem des Warenhandels“. Auch seien die Belastungen nicht abzusehen ${ }^{147}$. Zwar war die BdL bereit, Genehmigungen für laufende Zahlungen des Kapitalverkehrs weitherzig zu erteilen, doch wollte sie in der Lage sein, die Genehmigungspraxis jederzeit restriktiver zu handhaben ${ }^{148}$. Erst seit Sommer 1953 zeichnete sich langsam eine Veränderung ihrer Haltung ab. Doch wenn die Bank nun auch einer Liberalisierung der Kapitalerträgnisse zustimmte, so wollte sie dadurch nicht die Kapitaleinfuhr fördern. Der Grund für ihren Meinungswandel war vielmehr, daß die EZU-Position einen solchen Schritt nahelegte und zum Teil sogar verlangte. Zusätzlich dazu war das Hindernis der Zahlungsbilanzposition weggefallen ${ }^{149}$. Der Vertreter der BdL, Eduard Wolf, begründete in einer Ressortbesprechung diesen Meinungsumschwung und legte dabei nach Einschätzung des Marshallplan-Ministeriums sogar ein "Schuldbekenntnis“ der Bank ab: „Die Bundesrepublik habe sich als extremer Gläubiger in der bisherigen Weigerung die laufenden Zahlungen des Kapitalverkehrs zu liberalisieren nicht richtig verhalten. Herr Dr. Wolf hatte sogar

OEEC betreffend die Liberalisierung des unsichtbaren Handels, TP (52) 31/17, 8.12.1952, zit. in BBK, B 330/3172, Behandlung laufender Zahlungen des Kapitalverkehrs in OEEC-Dokumenten, 2.1.1953.

${ }^{143}$ BBK, B 330/2012, Erhard an Berg, 10.11.1952; BAK, B 102/56 905, V (Schöne), Lockerung bzw. Beseitigung der Devisenzwangswirtschaft, 24.6.1952.

${ }^{144} \mathrm{Vgl}$. BAK, B 102/11 579.1, I A 1 c (Weigel), Vermerk zum wissenschaftlichen Beirat, 25.11.1952.

${ }^{145}$ Vgl. BBK, B 330/2055, Direktorium, Protokoll, 9.10.1952.

146 Vgl. BBK, NL Emminger, Vermerke zur Konvertibilität, Emminger an von Mangoldt, 14.2.1954 und 23.3.1954.

147 BBK, B 330/2011, Vocke an Abs, 2.5.1953; BBK, B 330/2015, Vocke an Blücher, 18.12.1952. Vgl. hierzu auch BAK, B 136/7864, Blücher an Erhard, 19.12.1952; BAK, B 102/55340, V C 1, Liberalisierung der laufenden Zahlungen des Kapitalverkehrs, 2.1.1953; BBK, B 330/3172, BMWi an BMM, 6. 1.1953; BMWi an BMF, 8.1.1953; BAK, B 102/55340, V C 1, Material zur Besprechung am 14.1.1953, 10.1.1953; BAK, B 136/7864, III/3, Vermerk für die Chef-Besprechung, 8. 5.1953; Haenlein an Adenauer, 9.5.1953; BBK, B 330/2011, Abs an Erhard, 2.5.1953.

148 Vgl. BAK, B 102/55333, BdL an BMM, 22.5.1953.

149 Vgl. BBK, B 330/2056, Direktorium, 22.6.1953. Vgl. auch BBK, B 330/2475, Emminger, Vermerk zum Unterausschuß Handelspolitischer Ausschuß, 18.6.1953. 
wörtlich ausgeführt, ein solches Verhalten von extremen Gläubigern trage dazu bei, den Bestand der EZU zu gefährden. ${ }^{150}$ Im weiteren Verlauf stimmte dementsprechend auch die BdL der Liberalisierung der Kapitalerträgnisse $\mathbf{z u}^{151}$. Ein wichtiger Grund für die Zustimmung der Bank war die EZU. Im Zentralbankrat wurde festgestellt, daß die Gegenseite ein "wichtiges Druckmittel“ in der Hand hatte: Zur gleichen Zeit wurde nämlich in der EZU die Gewährung einer Rallonge verhandelt ${ }^{152}$.

Wenngleich eingeräumt werden muß, daß der europäische Kapitalmarkt weder so attraktiv noch so ergiebig wie der amerikanische Kapitalmarkt war, zeigt die Haltung der BdL zur Frage der Liberalisierung der Kapitalerträgnisse im OEEC-Rahmen, daß die Funktion der Konvertibilität, den Kapitalimport zu ermöglichen, für sie keine Rolle spielte. Anfänglich schätzte sie die negativen Aspekte des Kapitalimportes, wie vor allem die Verwundbarkeit und die unter Umständen auftauchenden Rückzahlungsschwierigkeiten zusammen mit den beträchtlichen Belastungen, als zu hoch ein. Später, schon im Oktober 1953, führte Vocke aus, daß die deutsche Wirtschaft Auslandskredite nicht mehr benötige: „Diese Frage (nach der Notwendigkeit des Kapitalimports, M.D.) wurde angesichts der Entwicklung unserer Devisenreserven und auch angesichts der Sparkapitalbildung in Deutschland von mir grundsätzlich verneint, ohne daß sich Widerspruch dagegen erhoben hätte. Ich sage grundsätzlich, denn natürlich können Einzelfälle denkbar sein, wo die Hereinnahme fremden Geldes erwünscht sein kann. Generell und grundsätzlich aber ist es nicht sinnvoll, heute sich um ausländisches Geld zu bemühen oder ausländisches Geld, falls es uns angeboten werden sollte, hereinzunehmen, uns also devisenmäßig zu verschulden, wo wir aus eigenen Kräften die erforderlichen Mittel ohne weiteres aufbringen können. ${ }^{153}$ Nur um der OEEC entgegenzukommen, um ihren Forderungen Genüge zu tun, und um den hohen deutschen EZU-Saldo abzubauen, stimmte sie der Liberalisierung der Kapitalerträgnisse im OEEC-Raum zu.

150 BAK, B 146/483, III/3, Vermerk zu V C 1, 29.7.1953. Vgl. auch BAK, B 102/56905, V C 1, Vermerk, 23.7.1953.

151 Allerdings ist festzuhalten, daß die BdL und das Finanzministerium auf dem Stichtag 15.Juli 1931 bestanden. Das grenzte den Berechtigtenkreis stark ein. Vgl. Runderlaß Außenwirtschaft Nr. 89/53 vom 30.9.1953, zit. in Kühne, Regelungen, S.311. Vgl. BAK, B 102/56905, V D, Lockerung der Devisenbewirtschaftung, 17.11.1953. Erst Ende des Jahres 1953 wurde diese Beschränkung aufgehoben.

152 BBK, B 330/72, ZBR, Stenogramm, 30.9.1952; BAK, B 102/55333, Seeliger, Vermerk zur 2. Rallonge, 12.10.1953.

153 BBK, B 330/2021, Vocke an Erhard, 24.10.1953 (Nicht abgegangen, da laut Präsident Vocke zunächst überholt). Vgl. hierzu auch Emminger, D-Mark, S. $69 f$., der seine Auseinandersetzung mit Vocke über die Frage, ob die Bundesrepublik ein „Kapitalüberschußland" sei, schildert. Laut Emminger habe Vocke noch im November 1953 die Bundesrepublik als kapitalarmes Land bezeichnet, das auf Kapitalimporte angewiesen sei. 


\section{Kreditierungen: Kosten der Nicht-Konvertibilität}

Die Tatsache, daß die DM und andere Währungen nicht konvertibel waren, brachte mit sich, daß der deutsche Außenhandel teils bilateral, teils regional organisiert war. Das führte dazu, daß die Bundesrepublik, die seit 1951/52 gegenüber dem EZU-Raum sowie gegenüber vielen bilateralen Verrechnungsländern Überschüsse erwirtschaftete, erhebliche Kredite gewähren mußte. Gegenüber der EZU hatte sie im Sommer 1952 eine kumulative Position von fast 500 Mio. RE aufzuweisen, was bedeutete, daß sie Kredite in Höhe von 320 Mio. Dollar vergeben hatte. Gegenüber der heterogenen Gruppe der bilateralen Verrechnungsländer waren Ende 1952 in vielen Fällen die vereinbarten Swings ausgeschöpft oder gar überschritten worden. Hier waren Kredite in Höhe von 185,2 Mio. Dollar aufgelaufen ${ }^{154}$. Da die Überschüsse sowohl zum EZU-Raum als auch zu den Verrechnungsländern anhalten konnten, mußte sich die BdL dem Problem weiterer Kreditvergaben stellen. Dabei mußte sie sich darüber klarwerden, welche Bedeutung sie den Exporten in diese Gebiete einräumen wollte bzw. welchen Stellenwert sie der Rückkehr zum Weltmarkt zusprach. Sie mußte diskutieren, ob beziehungsweise inwieweit diese Kredite als Kosten $z u$ bewerten waren, und ob beziehungsweise inwieweit sie diese Kosten tragen wollte.

Bilaterale Verrechnungsländer: principiis obsta

$\mathrm{Da}$ sich die deutschen Exportbemühungen - vehement unterstützt vom Wirtschaftsministerium - auch auf die Verrechnungsländer erstreckten und da viele dieser Länder wegen ihrer Dollarknappheit versuchten, Importe vor allem aus Ländern außerhalb des Dollarraums zu beziehen, wuchs auch der deutsche Export in diese Gebiete überproportional an ${ }^{155}$. $\mathrm{Da}$ aber die deutschen Importe aus diesen Regionen hinter den deutschen Exporten zurückblieben, waren im Rahmen des Bilateralismus Schwierigkeiten vorprogrammiert. Im Sommer 1952 traten diese massiv mit Brasilien auf. Damit wurde Brasilien im Jahre 1952 zu einem ersten Testfall ${ }^{156}$.

154 Vgl. BdL, Geschäftsbericht 1952, S.73. Die Bundesrepublik hatte Ende 1952 bilaterale Abkommen mit Ägypten, Argentinien, Brasilien, Bulgarien, Chile, Kolumbien, Ecuador, Finnland, Iran, Japan, Jugoslawien, Paraguay, Polen, Spanien, Tschechoslowakei, Ungarn, Uruguay.

155 Der deutsche Export wuchs von 1136 Mio. \$ (1949) auf 4043 Mio. \$ (1952). Der deutsche Export in die bilateralen Verrechnungsländer wuchs von 73 Mio. \$ (1949) auf 647 Mio. \$ (1952). Vgl. BdL, Geschäftsbericht 1952, S.67. Für die im Wirtschaftsministerium vertretenen Anschauungen vgl. Erhard, Rückkehr, S.160ff.

156 Jugoslawien, das Anfang 1952 seinen Swing ausgenutzt hatte und um eine Swingerhöhung nachsuchte, war der erste Problemfall. Da aber das jugoslawische Ersuchen um weiteren Kredit aus politischen Gründen von den USA und der Alliierten Hohen Kommission unterstützt wurde, war es ein Sonderfall. Vgl. BBK, B 330/52, AHK, Memorandum, 28.11.1951; ZBR, Protokoll und Stenogramm, 7./8.2.1952 samt Anlagen. Vgl. auch BBK, B 330/55, ZBR, Protokoll, 17.4.1952; BBK, B 330/56, ZBR, Protokoll, 30.4.1952; BBK, B 330/2015, Brown (US Treasury) an Vocke, 31.3.1952; Vocke an Brown, 3.4.1952. 
Tabelle 8: Außenhandel Brasilien - BRD 1929, 1938, 1950-1955

\begin{tabular}{lrrrcc}
$\begin{array}{c}\text { bras. Einfuhr } \\
\text { total } \\
\text { Mio.\$ }\end{array}$ & $\begin{array}{c}\text { bras. Einfuhr } \\
\text { aus BRD } \\
\text { Mio.\$ }\end{array}$ & $\begin{array}{c}\text { bras. Ausfuhr } \\
\text { total } \\
\text { Mio.\$ }\end{array}$ & $\begin{array}{c}\text { bras. Ausfuhr } \\
\text { nach BRD } \\
\text { Mio. \$ }\end{array}$ & $\begin{array}{c}\text { Anteil Brasilien an } \\
\text { deutschem Export } \\
\%\end{array}$ \\
\hline 1929 & 416,0 & 52,8 & 455,2 & 39,9 & 1,6 \\
1938 & 295,4 & 73,8 & 295,6 & 56,4 & 2,9 \\
1950 & 1085,1 & 18,8 & 1355,5 & 18,3 & 1,8 \\
1951 & 1987,1 & 110,7 & 1769,0 & 84,7 & 3,2 \\
1952 & 1986,0 & 183,8 & 1418,1 & 80,0 & 3,8 \\
1953 & 1318,7 & 108,3 & 1539,3 & 147,1 & 2,5 \\
1954 & 1633,5 & 157,1 & 1561,8 & 187,5 & 2,7 \\
1955 & 1306,8 & 88,0 & 1423,3 & 104,4 & 1,2 \\
\hline
\end{tabular}

Quelle: Sp. 1-4: Statistisches Bundesamt, Fachserie G, Außenhandel Reihe 8, Außenhandel des Auslandes, Brasilien Nr.201, 1960, S.10; Sp.5: berechnet nach Statistisches Jahrbuch für das Deutsche Reich bzw. für die Bundesrepublik Deutschland, div. Jahrgänge.

Wie Tabelle 8 zeigt, verdoppelte Brasilien innerhalb des Jahres 1951 seinen Import bei nur geringfügig ansteigendem Export. Als Ergebnis war auch der deutsche Export nach Brasilien sprunghaft angestiegen. Dabei hatte die deutsche Wirtschaft den scharf umkämpften brasilianischen Markt insoweit erobert, als sie den deutschen Lieferanteil von 1,7 Prozent (1950) auf 5,6 Prozent (1951) und schließlich auf 9,3 Prozent (1952) gesteigert hatte. Damit rangierte im Jahre 1952 der brasilianische Markt in der Wichtigkeit für den deutschen Export an neunter Stelle. Die Konsequenz der brasilianischen Importsteigerung war, daß der bisherige deutsche Schuldsaldo abgebaut wurde. Bei weiteren brasilianischen Importen war der im Rahmen des deutsch-brasilianischen Handels- und Zahlungsabkommen vereinbarte Kredit in Höhe von 13,5 Mio. Dollar im April 1952 ausgeschöpft.

Als sich im Verlauf des Frühjahrs 1952 zeigte, daß Brasilien nicht in der Lage war, den über den Swing hinausgehenden Betrag in Gold oder Dollar abzudekken, wurde die Kreditlinie für Brasilien im Juni auf 23 Mio. Dollar erhöht, denn auf deutscher Seite bestand ein starkes Interesse am Export nach Brasilien. Doch auch diese Erhöhung um circa 70 Prozent erwies sich schon bald als unzureichend. Am 18. Juli wies das Brasilien-Konto einen Schuldsaldo von 35,7 Mio. Dollar auf ${ }^{157}$. Da die Verhandlungen einer nach Brasilien entsandten deutschen Delegation ergebnislos verliefen, Brasilien jedoch nicht willens war, seine Importe zu drosseln, verfestigte sich innerhalb der Bundesrepublik die Ansicht, daß nun deutsche Maßnahmen unabweisbar seien. Allerdings erwies es sich als schwierig, konkrete, durchgreifende und schnell wirkende Schritte zu beschließen ${ }^{158}$.

157 Vgl. BBK, B 330/57, ZBR, Stenogramm, 25.6.1952; BBK, B 330/60, Ländersachbearbeitung, Vermerk Brasilien, 24.7.1952.

158 Vgl. BBK, B 330/60, Ländersachbearbeitung, Vermerk Brasilien, 24.7.1952. Verschiedene kleinere Maßnahmen wurden durchgeführt. So wurde am 15.5.1952 die Einfuhr für brasilianische Landes- 
Diese Situation ließ der Zentralbank kaum Optionen. Sofern sie dem kontinuierlichen Ansteigen des Schuldsaldos nicht tatenlos zusehen wollte, konnte sie der Entwicklung nur durch devisenpolitische Vorkehrungen Einhalt gebieten. Nachdem Ende August 1952 der brasilianische Schuldsaldo eine Höhe von 72 Mio. Dollar erreicht hatte, beschloß der Zentralbankrat Anfang September, die Zahlungsaufträge des Banco do Brasil an die deutschen Exporteure nicht mehr durch Umtausch in DM, sondern nur noch durch Gutschrift der Währungsbeträge zu erfüllen ${ }^{159}$. Den Brasilien-Exporteuren wurde erlaubt, diese Gutschriften in Verrechnungsdollar (Brasilien) entweder zu einem späteren Zeitpunkt einzulösen oder sie auf dem freien Markt an Brasilien-Importeure zu verkaufen. Den Brasilien-Importeuren war gleichzeitig gestattet worden, ihre Zahlungsverpflichtungen zu 80 Prozent durch frei erworbene und billigere Verrechnungsdollar (Brasilien) zu begleichen ${ }^{160}$.

Obwohl dieser Beschluß Ausdruck einer Zwangslage war, erregte er beträchtliches Aufsehen und einigen Unwillen. Das Wirtschaftsministerium wies die Bank auf die Bedeutung der Ausfuhr für die deutsche Wirtschaft hin. Trotz aller Befürwortung der Konvertibilität hob es die Notwendigkeit des Handelsaustausches mit den Verrechnungsländern im allgemeinen und Brasilien im besonderen hervor. Es machte sich zum Anwalt der Exportindustrie, die in seinen Augen hohen Risiken ausgesetzt und enormen Belastungen unterworfen war ${ }^{161}$. Dagegen blieb die BdL bei ihrem schon früher gelegentlich formulierten Standpunkt, daß die Zentralbank der Exportwirtschaft nicht alle Risiken abnehmen könne. Sie rückte die Kosten des Bilateralismus in den Mittelpunkt ${ }^{162}$. Dabei wies sie darauf hin, daß hohe Kreditvergaben zu einer Geldvermehrung führen und demnach „nicht ohne Einfluß auf die eigene Währungspolitik" $\operatorname{sind}^{163}$. Des weiteren führte sie aus, daß die aufgelaufenen Forderungen "wirklich nicht vollwertig“ seien und daß ein weiteres Entgegenkommen eine Umlenkung des Exportes von Hartwährungs- nach Weichwährungsländern mit sich bring $e^{164}$. Als Bank - darauf bestand sie - müsse sie bankpolitische Grundsätze befolgen, vor allem dürfe sie nicht „gutes Geld dem schlechten nachwerfen " ${ }^{\text {"165 }}$. Auch könne sie keine langfristigen

produkte freigegeben. Das führte aber zu keiner nennenswerten Steigerung der deutschen Importe aus Brasilien.

$159 \mathrm{Ab}$ sofort wurden alle neuen Zahlungsaufträge nur noch in Verrechnungsdollar (Brasilien) gutgeschrieben. Zahlungsaufträge, die vor dem 4.9.1952 abgeschlossen worden waren, wurden zur Hälfte in DM, zur Hälfte in Verrechnungsdollar (Brasilien) abgegolten.

160 Vgl. BBK, B 330/60, ZBR, Protokoll, 2./3.9.1952. Die prozentuale Begrenzung sollte zum Abbau des brasilianischen Schuldsaldos bei der BdL führen.

161 Vgl. BBK, B 330/2021, von Maltzan an BdL, 15.9.1952.

162 Vgl. BBK, B 330/2021, Erhard an Vocke, 14.12.1951; Vocke an Erhard, 19.12.1951; Vocke an Erhard, 15.1.1952. Vgl. auch BBK, B 330/60, ZBR, Protokoll, 2./3.9.1952; Tüngeler, „Die Neuregelung des Zahlungsverkehrs mit Brasilien“, in: Handelsblatt, 17.9.1952, S.6.

163 BBK, B 330/2021, BdL an Erhard, 22. 9. 1952. Im Frühjahr 1953 sprach die BdL anläßlich des Spanien-Falles sogar von "einer wirklichen währungspolitischen Gefahr ${ }^{\alpha}$. Vgl. BBK, B 330/69, BdL an BMWi, 3.6.1953.

164 BBK, B 330/60, ZBR, Stenogramm, 2./3.9.1952; BBK, B 330/64, ZBR, Stenogramm, 7.1.1953.

165 So Vocke anläßlich der argentinischen Zahlungsschwierigkeiten, BBK, B 330/54, Vocke an BMWi, 15.2.1952. 
Kredite vergeben - schon gar nicht an ausländische Staaten. Bernard formulierte deshalb knapp und bündig: „principiis obsta. “166 Darüber hinaus lehnte die Bank auch eine Übernahme des Risikos auf den Bund aus volkswirtschaftlichen sowie aus währungspolitischen Gründen ab, denn dadurch würde nur schrittweise ihre Position in Außenhandelsfragen unterminiert werden ${ }^{167}$. Deshalb befürwortete sie, den Export nach Brasilien zurückzuschrauben. Sie hob hervor, in einer solchen Situation sei es einfach unausweichlich, den Export zu treffen. Angesichts der Kosten der Kreditierungen - und die bilateralen Kredite waren für die BdL eindeutig Kosten - war das für sie die einzige Möglichkeit ${ }^{168}$. Die deutsche Exportwirtschaft mußte laut BdL die entsprechenden Konsequenzen tragen. Während die Bank noch im Geschäftsbericht 1950 die Vorteile des Exportes nach Übersee herausgestellt hatte, nahm sie nun davon eindeutig Abstand ${ }^{169}$.

EZU-Rallongen: principiis noli obstare

Während im Sommer 1952 „erschreckende Zugänge“ auf dem Brasilien-Konto zu verzeichnen waren, verbesserte sich die deutsche Zahlungsbilanz zum EZURaum "weiterhin in beängstigendem Rhythmus"170. Die Bundesrepublik hatte im Sommer 1952 ihre Quote bei der EZU fast ausgeschöpft, so daß neue Vereinbarungen notwendig wurden. Bisher hatten sich die EZU-Mitglieder bei ähnlich gelagerten Problemen - wie zum Beispiel im belgischen Fall - auf Rallongen, also ein Aufstocken der Quote, geeinigt. Diese Quotenaufstockungen wurden entweder anteilmäßig in Gold ausbezahlt oder mit einer Abschlagszahlung entgolten. In beiden Fällen mußte das Gläubigerland einen gewissen Teil seiner Überschüsse kreditieren.

In der BdL traf eine weitere Kreditvergabe an die EZU im Sommer 1952 vorerst auf wenig Gegenliebe. Sie hoffte, ein Überschreiten der eingeräumten Quote vermeiden zu können, und hielt jegliche Diskussion über eine Rallonge „für verfrüht“. Dabei warnte sie vor „einer der wirtschaftlichen Vernunft widersprechen-

166 BBK, B 330/60, ZBR, Stenogramm, 2./3.9.1952. Diese Meinung wurde nicht von allen Zentralbankratmitgliedern geteilt. Könneker wollte z.B. die handelspolitischen Aspekte berücksichtigt wissen.

167 Vgl. BBK, B 330/63, ZBR, Stenogramm, 17./18.12.1952; BBK, B 330/2055, Direktorium, Protokoll, 9.10.1952. Auch als der Zentralbankrat im Sommer 1954 die Konsolidierung der EZU-Kredite diskutierte, wurde die Übernahme der konsolidierten Beträge in eine Verrechnungskasse vorgeschlagen. York Hoose, Präsident der Landeszentralbank Niedersachsen, warnte davor mit folgenden Worten: „Dann nimmt man uns auch die Verfügung über die Gold- und Devisenbestände und wir haben den Zustand, daß die Notenbank völlig ausgeschaltet ist von allem, was mit dem Außenhandel zu tun hat. Ich warne sogar davor, diesen Gedanken weiter zu verfolgen, die ganze EZU-Schuld in eine Verrechnungskasse hineinzunehmen. Das ist der Anfang vom Ende und das Ende sehe ich darin, daß wir überhaupt ausgeschaltet werden von den Außenhandelsfragen." In: BBK, B 330/78, ZBR, Stenogramm, 16.6.1954.

168 Vgl. BBK, B 330/64, ZBR, Stenogramm, 21./22.1.1953, wo Vocke feststellt: „Der Export wird immer gedrosselt, wenn von einem Land nicht mehr gezahlt wird."

169 Vgl. BdL, Geschäftsbericht 1950, S.49.

170 BBK, B 330/60, Ländersachbearbeitung, Vermerk Brasilien, 24.7.1952; BAK, B 146/565, Sachs, Vermerk, 21.5.1952. 
den übermäßigen Kreditgewährung an andere EZU-Länder “171. Wie schon bei den bilateralen Verrechnungsländern führte die BdL bankgesetzliche Gründe an. Sie drohte sogar damit, „daß sie [. . .] keine weiteren Kreditierungen vornehmen könne und den deutschen Exporteur an den Finanzminister verweisen müsse ${ }^{\text {"172 }}$.

Auf den ersten Blick scheint sich demnach die Haltung der BdL zu weiteren Kreditierungen an die EZU nicht von ihrer Position zu bilateralen Swingkrediten zu unterscheiden. Das ist aber nur eingeschränkt korrekt. Zum ersten ist festzuhalten, daß diese Aussagen in die Auseinandersetzung zwischen Erhard und der BdL über die Konvertibilitätsfrage und die Erhöhung der Liberalisierung einzuordnen sind. Sie sind Drohgebärden, die Erhard dazu veranlassen sollten, einer Erhöhung der deutschen Liberalisierung zuzustimmen. Das macht vor allem die Tatsache deutlich, daß die BdL in anderen Zusammenhängen und vor dem EZU-Forum weitere Kreditierungen, unter Umständen und vorübergehend sogar bis zu 100 Prozent, in Aussicht stellte. Sie stimmte im weiteren Verlauf wenn sie auch manchmal Unwillen zeigte - Erhöhungen der Kreditierungsverpflichtungen stets ohne größere Einwände $\mathrm{zu}$ erheben $\mathrm{zu}^{173}$. Zum zweiten ist $\mathrm{zu}$ konstatieren, daß die anfänglich formulierte BdL-Opposition gegen weitere Kreditierungen im Verlauf der Zeit aus verschiedenen Gründen schwächer wurde.

Ein erster Grund für die Abschwächung der Opposition war, daß Rallongen wie sich bald herausstellte - mit Goldzahlungen verbunden waren. Dieses Gold konnte den Währungsreserven zugeführt werden. Wenn die Bank auch schon im Sommer 1953 meinte, Goldzahlungen seien eigentlich nicht mehr notwendig, so waren sie in der Anfangszeit ein wichtiger Aspekt ${ }^{174}$. Ein weiteres Motiv war, daß die an die EZU gewährten Kredite nicht wertlos waren. Vielmehr konnten sie im gesamten EZU-Raum einschließlich der abhängigen Gebiete verwendet werden. Diese Kredite mußten somit nicht als Kosten verbucht werden. Vielmehr konnten die aufgelaufenen EZU-Guthaben in gewisser Hinsicht als Währungsreserven gelten, da sie vor allem für Rohstoffbezüge aus dem SterlingRaum benutzt werden konnten. In einer Ressortbesprechung stellte der Vertreter der BdL klar, „im übrigen [seien] auch die weiterhin an die EZU kreditierten Überschüsse keineswegs ein Vermögensentzug ohne Gegenwert, da die hierfür

171 BAK, B 102/6428, Elson, Niederschrift über die Ressortbesprechung am 1.7.1952, 3.7. 1952; BBK, NL Emminger, Konvertibilität/Abbau der Devisenzwangswirtschaft I 1949-52, Emminger, Bemerkungen hinsichtlich der deutschen Stellungnahme zur Liberalisierung und Konvertibilität im Rahmen der OEEC/EZU, 10.9.1952.

172 BAK, B 102/6428.1, V C 1 an von Maltzan, 5.9.1952. Daß eine solche Politik für die Bank keine ernsthafte Option war, ergibt sich aus Fußnote 167.

173 Vgl. BBK, B 330/1313, Treue, Behandlung der Stellung Deutschlands in der EZU, 17.7.1952. Vgl. auch BAK, B 146/527, von Mangoldt an BMM, 24.7.1952; BBK, B 330/2056, Direktorium, Protokoll, 3.10.1953.

${ }^{174}$ Im EZU-Abkommen war nicht festgelegt, daß Rallongen mit Goldzahlungen verbunden waren. Im Sommer 1952 war das auch noch kein fest verankertes Prinzip. Als die BdL im Sommer 1952 einer Rallonge zustimmen mußte, war ihre erste Stellungnahme, daß sie nicht „ohne weiteres“ auf Goldzahlungen verzichten könne. Vgl. BAK, B 102/5428, Elson, Niederschrift über die Ressortbesprechung am 1.7.1952, 3.7.1952. Vgl. hierzu auch S. $160 \mathrm{ff}$. 
eingetauschten Forderungen an die EZU, wenn auch nicht vollwertige Devisen wie Gold oder Dollar, so doch aller Voraussicht nach recht nützliche Währungsreserven darstellten, da sie in einem sehr großen Bereich des Außenhandels (rund $70 \mathrm{v}$. H.) jederzeit multilateral für fast alle von uns benötigten Waren einsetzbar seien (im Gegensatz zu den nur sehr beschränkt und nur für überteuerte Waren einsetzbaren Brasilien-Forderungen u. ä.) ${ }^{\alpha 175}$. Ein dritter Grund für die Haltung der BdL war, daß der Handelsaustausch mit Europa zu wichtig war. Während der. Export in die bilateralen Länder bei Zahlungsunfähigkeit eben zwangsläufig eingeschränkt werden müsse, könne - so stellte die BdL heraus der Export in die EZU-Länder keinesfalls gedrosselt werden. Die Bank befürchtete, daß eine Begrenzung der EZU-Kreditlinien ,im Augenblick doch bedenkliche kontraktive Wirkungen für den Handel zwischen den EZU-Ländern zur Folge habe "176. Deshalb befürwortete und gewährte sie weitere Kredite an die EZU. $\mathrm{Da}$ ihres Erachtens die OEEC und ihr Liberalisierungsprogramm beibehalten und gestärkt werden mußten, wollte sie mit diesen Kreditvergaben „das Unsrige tun $^{“ 177}$. Die Kredite an die EZU waren laut BdL der „Preis für die Aufrechterhaltung der Liberalisierung ${ }^{\prime 178}$.

Insgesamt ist festzuhalten, daß die BdL deutlich zwischen bilateralen und EZUKrediten unterschied. Im Gegensatz zum Wirtschaftsministerium, das alle Kreditierungen als "unfreiwilligen oder unbewußten Kapitalexport", als „staatliche Zwangskredite" und „Vermögensentzug" für ein "kapitalarmes Land" ansah" ${ }^{179}$, war die BdL weit davon entfernt, diese beiden Kreditvergaben als gleichartig zu verstehen. Ganz im Gegenteil: Je offensichtlicher die Schwierigkeiten des Bilateralismus wurden, desto mehr wuchs ihre Angst vor einem Rückfall in den Bilateralismus auch in Europa und desto mehr sah sie die positiven Aspekte der EZU und der EZU-Kredite. Diese waren notwendig, sinnvoll und übernahmen wichtige Funktionen. Darüber hinaus waren sie verwertbar. Angesichts dieses Nutzens mußten ihre Begleiterscheinungen wie eine Verflüssigung des Geldmarktes ver-

175 BBK, NL Emminger, Konvertibilität/Abbau der Devisenzwangswirtschaft II 1953, BdL, Vermerk über die zweite Ressortbesprechung mit Herrn Professor Lutz über die Probleme der Konvertierbarkeit der Währungen, 14.8.1953. Vgl. auch BBK, B 330/63, Bernard an Blücher, o.D. (11.12.1952); BBK, B 330/1313, Vocke an Merton, 15. 8. 1952.

176 BBK, B 330/2012, Vocke an Erhard, 19.3.1953. Auch aus binnenwirtschaftlichen Gründen war eine Begrenzung der Kreditlinien laut BdL bedenklich, denn es sei mit Produktionsschrumpfung und mit sekundären deflationistischen Tendenzen zu rechnen. Vgl. BBK, NL Emminger, Konvertibilität/Abbau der Devisenzwangswirtschaft II 1953, BdL, Vermerk über die zweite Ressortbesprechung mit Herrn Professor Lutz über die Probleme der Konvertierbarkeit der Währungen, 14.8.1953.

177 BBK, B 330/2012, Vocke an Erhard, 11.12.1952.

${ }^{178}$ BBK, NL Emminger, Konvertibilität/Abbau der Devisenzwangswirtschaft II 1953, Emminger, Zum Thema Konvertibilität, 8.4.1954.

179 Vgl. BAK, B 102/55333, Müller-Armack, Diskussionsbeitrag zum Thema Exportförderung, 20.6. 1953; BAK, B 102/12651, I A 1, Ausarbeitung, 27.8.1953; BBK, NL Emminger, Konvertibilität/Abbau der Devisenzwangswirtschaft II 1953, BdL, Vermerk über die zweite Ressortbesprechung mit Herrn Professor Lutz über die Probleme der Konvertierbarkeit der Währungen, 14.8.1953. 
nachlässigt werden ${ }^{180}$. So führte der in einer nicht-konvertiblen Welt existierende Zwang zu Kreditierungen nur zu einer größeren Wertschätzung der regionalen Zwischenformen wie der EZU, und die BdL sah noch deutlicher die positiven Seiten des Regionalismus. Die Konsequenzen dieser Haltung, wie die Stagnation oder gar der Rückgang der deutschen Exporte in die bilateralen Länder, sah die Bank nicht als Problem an. Aus bank- und handelspolitischen Gründen nahm sie diese Konsequenzen in Kauf und schätzte den Erhalt und den Ausbau des europäischen Marktes als weitaus wichtiger ein als die Rückkehr zum Weltmarkt.

\section{Die Europäische Zablungsunion: wachsende Kosten?}

Sowohl während der Gründungsverhandlungen als auch anläßlich der belgischen Gläubigerkrise hatte die BdL das Prinzip einer regionalen Zahlungsunion befürwortet. In ihren Augen überwog der Nutzen deutlich die Kosten. Die EZU hatte die Aufnahme der europäischen Liberalisierung abgesichert und dadurch zum Aufbau des innereuropäischen Handels beigetragen. Darüber hinaus hatte sie der Bundesrepublik während der Koreahausse einen zusätzlichen Kredit gewährt. Trotz dieser positiven Seiten wurden aber im Laufe der Zeit auch die Kosten des Regionalismus deutlicher. Abgesehen von dem Verzicht auf Kapitalimporte und abgesehen von dem letztendlich bereitwillig geleisteten Tribut in Form der Kreditvergabe an die EZU, war die Mitgliedschaft in der EZU mit Einschnitten in die Währungsautonomie und mit einem kontinuierlichem Goldverzicht verbunden. Darüber hinaus offenbarten die bis dato geschätzten handelspolitischen Auswirkungen - wie z. B. die Umlenkung des deutschen Imports vom Dollarraum auf den EZU-Raum - mit erstarkender deutscher Zahlungsbilanzposition und schwindender Notwendigkeit ihre negativen Seiten wie die Schaffung von Abhängigkeiten, höhere Kosten und dadurch möglicherweise eine Schmälerung der Wettbewerbsfähigkeit. Inwieweit sah die BdL diese Konsequenzen der EZU als Kosten an? Inwieweit war sie bereit, diese Kosten zu tragen?

Kosten I: Einschränkung der Souveränität

Während der belgischen Gläubigerkrise hatte sich die BdL für eine Stärkung der EZU und ihrer supranationalen Aspekte ausgesprochen. Vor allem die Gläubiger sollten zur Korrektur ihres Zahlungsbilanzungleichgewichtes angehalten werden. Das aber war ein zweischneidiges Schwert. Im März 1952 hatte die Bundesrepublik die erste Tranche als Gläubiger überschritten. Im Sommer 1952 stand sie kurz vor der Erschöpfung ihrer Quote in Höhe von 500 Mio. RE. Ende 1953

180 Während die BdL in den bilateralen Krediten eine währungspolitische Gefahr sah, versicherten BdL-Vertreter das Wirtschaftsministerium in bezug auf die EZU-Kredite, daß diese währungspolitisch nicht gefährlich seien. Vgl. BBK, NL Emminger, Konvertibilität/Abbau der Devisenzwangswirtschaft II 1953, BdL, Vermerk über die zweite Ressortbesprechung mit Herrn Professor Lutz über die Probleme der Konvertierbarkeit der Währungen, 14.8.1953; BBK, B 330/69, BdL an BMWi, 3.6.1953. 
belief sich ihre kumulative Rechnungsposition auf 822,2 Mio. RE, mit steigender Tenden $z^{181}$. Damit hatte sich die Bundesrepublik vom normalen zum extremen Gläubiger entwickelt, was zur Folge hatte, daß die EZU immer wieder die deutsche Situation und Politik diskutierte und wirtschaftspolitische Empfehlungen aussprach.

Erste Untersuchungen der deutschen Gläubigerposition fanden im Juli und im September 1952 statt. Als Resultat forderte das EZU-Direktorium eine Erhöhung der deutschen Liberalisierung. Zur Kreditpolitik bezog es nur am Rande und sehr moderat Stellung ${ }^{182}$. Bei der BdL trafen die Stellungnahmen des EZUDirektoriums auf Zustimmung, denn auch sie befürwortete eine weitere Liberalisierung. Darüber hinaus war der Zentralbankrat seit Ende April 1952 überzeugt, die interne konjunkturpolitische Situation und die außenwirtschaftliche Entwicklung würden kreditpolitische Erleichterungen erlauben. Deshalb hatte er eine weniger restriktive Politik eingeläutet ${ }^{183}$. Auf eine Anfrage der OEEC konnte die Bank demnach im Oktober 1952 postwendend antworten, daß sie die Diskont- und Mindestreservesätze schon gesenkt habe und keine restriktive Politik beabsichtige ${ }^{184}$. Insgesamt befand sich also die Bank im Jahre 1952 im Einvernehmen mit der OEEC. Hinsichtlich des Souveränitätsverzichtes konnte Emminger noch im Dezember 1952 fordern, daß „einerseits die internationalen Gremien, welche die Wirtschaftspolitik der Mitgliedsländer zu verfolgen und überwachen in der Lage sind, stärkeren Einfluß auf diese enthalten, und andererseits und vor allem, daß die Mitgliedsländer selbst freiwillig auf einen Teil ihrer währungspolitischen Autonomie zugunsten der Aufrechterhaltung ihres äußeren Gleichgewichts verzichten, weil sie von dem Wert und Nutzen einer solchen wirtschaftspolitischen Einstellung für ihre eigenen Interessen und die gemeinsamen Interessen aller Partnerländer überzeugt sind ${ }^{\text {“185. }}$.

Auch in den Jahren 1953 und 1954 hielten die deutschen Überschüsse an, weshalb die Pariser Gremien auch in dieser Periode die deutsche Wirtschafts- und Währungspolitik aufmerksam verfolgten. Dabei kamen sie mehr und mehr auf die Liberalisierung der Kapitalerträgnisse, die Kreditpolitik und sogar auf den Wechselkurs der DM zu sprechen. Damit thematisierten sie Bereiche, die im Ressort der BdL lagen. Daher verwundert es nicht, daß dies auch manchmal auf Ab-

${ }^{181}$ Vgl. BdL, Geschäftsbericht 1954, S. 98.

182 Vgl. BAK, B 146/527, Germania Paris an BMM, 24.7.1952; BBK, NL Emminger, Deutschland als EZU-Gläubiger, OEEC/EPU, Examination of Germany, MBC (52)62, 30.9.1952.

${ }^{183} \mathrm{Am}$ 17.4.1952 wurden Rediskontkontingente festgesetzt. Am 30.4.1952 wurden die Mindestreservesätze nach der Höhe des Einlagenbestandes gestaffelt. Das bedeutete eine Entlastung für kleinere Institute und senkte das Reserve-Soll um ca. 250 Mio. DM. Am 28.5.1952 wurde der Diskont von 6 auf 5\% gesenkt. Am 20.8.1952 erfolgte eine weitere Senkung auf 4,5\%. Zum Verlauf der Beschlußfassungen vgl. die entsprechenden Protokolle in BBK, B 330/55 ff.

${ }^{184} \mathrm{Vgl}$. BBK, B 330/1310, BdL an Vertretung der Bundesrepublik Deutschland bei der OEEC, 2.10.1952.

185 BBK, NL Emminger, Konvertibilität/Abbau der Devisenzwangswirtschaft I 1949-52, Emminger, Konvertibilität und Liberalisierung, 15.12.1952. 
wehr bei der BdL traf. Bei einer Gelegenheit führte schon allein die Tatsache, daß im OEEC-Forum Maßnahmen angesprochen wurden, die die BdL ablehnte, zu erheblichem Unmut ${ }^{186}$. Das war aber eine Ausnahme, und diese Unstimmigkeit konnte leicht beigelegt werden, denn selbst im Frühjahr 1954, als die Bundesrepublik eine kumulative Position von über 1000 Mio. RE erreicht hatte, waren die Empfehlungen der Pariser Gremien noch immer „verhältnismäßig zahm “187. Die zur Untersuchung der europäischen Ungleichgewichte eingesetzte Arbeitsgruppe Nr.8, die unter anderem auch die deutsche Position prüfte, sprach die Möglichkeiten einer DM-Aufwertung und einer Kreditexpansion lediglich an. Letztendlich nahm sie aber von einer entsprechenden Empfehlung Abstand. Sie betonte vielmehr, daß „alle Maßnahmen [...] so beschaffen sein sollten, daß nicht für Deutschland das Dollarproblem auftaucht und die Konvertibilität verzögert wird ${ }^{\alpha 188}$. Auch sah die OEEC von der Empfehlung ab, den deutschen Export zu drosseln. Vor allem aber formulierte sie kein Junktim zwischen der EZUVerlängerung und deutschen Maßnahmen gegen die Überschüsse ${ }^{189}$. Darüber hinaus herrschte auch bei dieser Gelegenheit hinsichtlich der Kreditpolitik prinzipielle Übereinstimmung zwischen der OEEC und der Zentralbank. Der Zentralbankrat sah sich in den Jahren 1953 und 1954 in der Lage, weitere kreditpolitische Lockerungen zu verfügen ${ }^{190}$. Ferner war die BdL selbst daran interessiert, die störenden Überschüsse abzubauen, da sie ein besseres Funktionieren der EZU und damit die Aufrechterhaltung der europäischen Liberalisierung anstreb$\mathrm{te}^{\mathrm{t} 91}$. Schließlich ist festzuhalten, daß die Empfehlungen der OEEC der BdL manchmal gelegen kamen, vor allem wenn sie im Rahmen von Auseinandersetzungen mit deutschen Stellen ihre Position stärkten ${ }^{192}$. So konnten die Stellungnahmen und Empfehlungen der EZU zur deutschen Wirtschaftspolitik die Wert-

${ }^{186}$ Im Frühjahr 1954 verwahrte sich Vocke vehement gegen von ihm befürchtete Empfehlungen der OEEC-Arbeitsgruppe 8, namentlich die einer Aufwertung. Vgl. BBK, B 330/1313, Vocke, Fernschreiben an Blücher, 12.3.1954.

${ }^{187}$ BBK, B 330/76, ZBR, Stenogramm, 31.3.1954.

${ }^{188}$ BBK, NL Emminger, Deutschland als EZU-Gläubiger, OEEC, Bericht der Arbeitsgruppe 8, $\mathrm{EC}(54) 4,5.3 .1954$.

189 Vgl. BBK, B 330/74, ZBR, Stenogramm, 16.12.1954; BBK, NL Emminger, Deutschland als EZUGläubiger, Gerbaulet an AA/BMW1/BdL, 15.4.1954; Emminger, Vermerk, 23.4.1954.

${ }^{190}$ Am 7.1.1953 wurde eine 0,5\% ige Diskontsenkung beschlossen; am 10.6.1953 wurde der Diskont auf 3,5\% und am 19.5.1954 auf 3\% gesenkt. Vgl. die Protokolle der entsprechenden Sitzungen des Zentralbankrates in BBK, B 330/64, B 330/69, B 330/77. Der Zentralbankrat kam sogar nach einer Grundsatzdiskussion überein, die aus den Überschüssen resultierende Liquidisierung gemäß den Regeln des Goldstandards zuzulassen. Vgl. hierzu die Diskussionen des Zentralbankrates am 1.4.1953, 15.4.1953, 30.4.1953 und 19.8.1953, in BBK, B 330/67f. und in BBK, B 330/71. Vgl. auch BBK, B 330/3379, Könneker, Mobilisierung von Ausgleichsforderungen, 10.8.1953; Wolf, Vermerk, 11.8.1953; BBK, B 330/3380, Wolf, Vermerk zu Wolff, 22.2.1954.

191 Vgl. BBK, B 330/74, ZBR, Protokoll und Stenogramm, 20.1.1954; BBK, B 330/76, ZBR, Protokoll und Stenogramm, 31.3.1954.

${ }^{192} \mathrm{Am}$ 23.3.1954 stellte Vocke in einem Brief an Erhard fest, daß es "nicht übel“" wäre, wenn die OEEC die völlige Freigabe der Sperrmarkguthaben innerhalb des EZU-Raums empfehlen würde. Damit hoffte er die Opposition von Finanzminister Schäffer zu überwinden. In: BBK, B 330/ 2012. Vgl. hierzu auch S. 191. 
schätzung der BdL für die EZU letztendlich nicht trüben. Wenn die BdL auch nicht mehr wie zuvor auf eine effektivere Kontrolle der extremen Gläubiger durch die EZU drängte, sondern von der Schaffung eines „übergeordneten Gremiums der Notenbanken " abriet und sich auch manchmal hinter der britischen Abneigung gegen die Supranationalität versteckte ${ }^{193}$, akzeptierte sie die bescheidenen Einschränkungen der Währungsautonomie im Rahmen der EZU. Derartige Einschränkungen standen selbst im Jahre 1954 nicht den Interessen der BdL entgegen, zumal auch im Rahmen eines anderen internationalen Währungssystems derartige Einschränkungen gegeben waren.

Kosten II: Goldverzicht und Reservenaufbau

Die Mitgliedschaft in der EZU implizierte, daß die Bundesrepublik bei anhaltenden Überschüssen Kredit gewähren mußte. Wie oben gezeigt wurde, stimmte die BdL diesen Krediten bereitwillig zu, denn im Vergleich zu den bilateralen Krediten waren sie mit Vorteilen verbunden. Gleichzeitig waren diese Kreditierungen aber auch mit Nachteilen verknüpft, namentlich dem Verzicht auf Gold- oder Dollarzahlungen. Das ging zu Lasten des Reservenaufbaus und setzte Grenzen bei der Liberalisierung zum Dollarraum. Die Wiederherstellung der traditionellen Handels- und Zahlungsbeziehungen - Ausgleich des Defizits zu Übersee durch Überschüsse in Europa - wurde dadurch erschwert.

Tatsächlich sah die Zentralbank in der Anfangszeit, im Sommer 1952, die Tatsache, daß möglicherweise keine Goldzahlungen für die deutschen Überschüssen über die EZU-Quote hinaus anfallen würden, als eine ungebührende Belastung an. Sie sah sich auf Goldzahlungen angewiesen und dazu berechtigt. Bei einem Reservenstand von 447,1 Mio. Dollar stellte Wolf im Juli 1952 in einer Ressortbesprechung über eine Rallonge heraus, daß die BdL nicht „ohne weiteres auf jede Goldzahlung verzichten könne“194. Auch der Zentralbankrat hob hervor, daß die Bundesrepublik „an sich“ einen Anspruch auf die volle Abgeltung der Überschüsse in Gold oder Dollar hätte ${ }^{195}$. Bei den Verhandlungen über eine Rallonge verzichtete die Bank jedoch ohne größere Einwendungen darauf, diesen Anspruch durchzusetzen. Trotz der laut BdL noch vorhandenen Dollarlücke und trotz der ihrer Meinung nach ungenügenden Reserven thematisierte sie letztendlich den Goldanteil der Rallonge nicht ${ }^{196}$.

Diese erste Stellungnahme blieb nicht von den sich Ende 1952 forcierenden Diskussionen über eine EZU-Reform unberührt, die das Problem der EZU-Härtung grundsätzlich ansprachen. Tatsächlich befürwortete im folgenden auch die BdL - anfangs gezwungenermaßen, später mehr und mehr überzeugt - eine Wei-

\footnotetext{
${ }^{193}$ Vgl. BBK, B 330/2012, Vocke an Erhard, 17.3.1952 und 19.3.1953.

194 BAK, B 102/6428, Elson, Niederschrift über die Ressortbesprechung am 1.7.1952, 3.7.1952.

195 BBK, B 330/60, ZBR, Stenogramm, 17./18.9.1952.

196 Vgl. BBK, B 330/1313, Treue, Vermerk, 17.7.1952.
} 
terentwicklung der EZU durch eine Härtung ${ }^{197}$. In den Ressortbesprechungen stellte sie sich den vor allem vom Marshallplan-Ministerium und der deutschen Delegation in Paris befürworteten Plänen zur schrittweisen Erhöhung der Goldanteils nicht entgegen. Maßgebend für diese Haltung war aber nicht so sehr der dadurch verringerte Goldverzicht; vielmehr waren es vor allem taktische Gründe, namentlich die Notwendigkeit, konsequent und stetig auf eine Weiterentwicklung der EZU in Richtung Härtung zu drängen, die die BdL, die OEECVertretung und das Marshallplan-Ministerium vereinten ${ }^{198}$. Demnach war dieses Übereinkommen auch fragil, so daß schon im März 1953 die Bundesregierung vor allem auf Insistieren der BdL - beschloß, die deutsche Forderung nach Härtung zwar "nachdrücklich“ zum Ausdruck zu bringen, aber gleichzeitig eine "elastische und keine ultimative" Position einzunehmen ${ }^{199}$. Auch im weiteren Verlauf standen für die BdL vor allem taktische Gründe im Vordergrund. Das führte dazu, daß sie die Forderungen nach Verringerung des Goldverzichts zwar immer vorbrachte, jedoch nicht darauf beharrte. Ein erster Grund dafür war, daß im Mai 1953 eine Alimentierung der Währungsreserven bei einem Stand von über 800 Mio. Dollar nicht mehr zwingend notwendig war. Ein zweiter Grund bestand darin, daß die BdL befürchtete, sie würde dadurch nur die eigene, noch zögerliche Position hinsichtlich der Liberalisierung der Kapitalerträgnisse erschweren ${ }^{200}$. Auch im Herbst 1953 befürwortete die Bank die Forderung nach Erhöhung des Goldanteils auf 65 Prozent bei Überschreitung der Quote, jedoch waren auch jetzt dabei vor allem taktische Erwägungen maßgebend. Der dadurch verringerte Goldverzicht spielte für die BdL dabei keine Rolle ${ }^{201}$. Wie Vocke schon im Sommer im Kabinettsausschuß festgestellt hatte, waren höhere Goldzahlungen nicht mehr notwendig. Ein Verzicht auf Gold stellte für die BdL keinen Kostenfaktor mehr dar. Vocke versicherte den Mitgliedern des Kabinettsausschusses: „Die Lage ist nicht alarmierend, denn schlimmstenfalls würden wir kein Gold erhalten, wenn wir unsere Gläubigerposition bei der EZU weiter erhöhen. “202

Warum die BdL trotz ihrer prinzipiellen Befürwortung einer EZU-Härtung letztendlich den Goldverzicht nicht als einen Kostenfaktor ansah, erklärt sich daraus, daß sie den Goldverzicht gar nicht als einen solchen verstand. Vocke stellte das etwas vereinfachend, überspitzt und die Wahrheit leicht verdrehend in einem seiner Plädoyers für die EZU folgendermaßen dar: „Unser Export

197 Vor die Alternative EZU-Reform oder neues Zahlungssystem gestellt, befürwortete der BdL-Vertreter im November 1952 in einer Ressortbesprechung unter erheblichen Vorbehalten eine Reform. Vgl. BAK, B 146/480, BMM, Protokoll über die Sitzung vom 14.11.1952, 17.11.1952.

198 Vgl. BAK, B 146/482, Schleiminger, Aufzeichnung über die Ergebnisse der 2. inoffiziellen Besprechung, 16.1.1953.

199 BAK, B 146/481, Schleiminger, Ergebnisbericht einer interministeriellen Besprechung, 17.3.1953.

$200 \mathrm{Vgl}$. BBK, NL Emminger, Deutschland als EZU-Gläubiger, Emminger, Vermerk, 28. 5. 1953.

201 Vgl. BBK, B 330/1313, Emminger, Vermerk, 16.10.1953; BBK, B 330/2852, Emminger, Vermerk, 24.10.1953.

202 BAK, B 136/7864, Kabinettsausschuß, 2.7.1953. 
nach den währungsschwächsten Ländern wie Griechenland usw. bringt uns auf der ganzen Linie Dollars, wenn auch nur zu 50 Prozent liquide Dollars. "203

Kosten III: Auswirkungen auf den Außenhandel

Abgesehen von den Beschränkungen der Währungsautonomie und dem Goldverzicht bringen Zahlungsunionen eine Umlenkung des Imports von billigeren zu teureren Produzenten mit sich. Gleichzeitig bietet eine Zahlungsunion der Exportindustrie Vorzugsmärkte und damit die Möglichkeit, sich nur auf diese Weichwährungsmärkte zu konzentrieren. Das kann den Zwang zu einem Ausbau der Wettbewerbsfähigkeit verringern, so daß eine Zahlungsunion langfristig - abgesehen von den effektiven Mehrkosten für Importe - Abhängigkeiten schaffen kann.

Diese problematischen Auswirkungen waren der BdL durchaus bekannt. Gleichwohl wurde in den ersten Jahren eine Handelsumlenkung für gut und notwendig geheißen. Die Verlagerung der Einfuhr verringerte die Dollarbelastung, während die Vorzugsmärkte Exportsteigerungen ermöglichten. Im weiteren Verlauf wurden aber zwangsläufig die negativen Seiten deutlicher. Bei wachsenden Währungsreserven war ein Abbau der Dollarbelastung nicht mehr zwingend notwendig, sondern unter Umständen nur teurer für die deutschen Importeure. Bei fortschreitender Rekonstruktion und offensichtlich schon hoher Wettbewerbsfähigkeit bedeutete die Existenz von Vorzugsmärkten, daß die Exportindustrie ihre Chancen in den Hartwährungsgebieten nicht wahrnahm.

Während der Wirtschaftsminister in den Diskussionen um die EZU stets diese negativen Aspekte einer Zahlungsunion hervorhob, standen diese problematischen Konsequenzen der EZU für die BdL nicht an erster Stelle. Zwar war sie der Meinung, daß das Vereinigte Königreich und Frankreich unter einer „bisher nicht beendeten Inflation leiden" und daß dort zu hohe Preise gälten ${ }^{204}$. Allzu viel Gewicht maß sie dieser Tatsache jedoch nicht bei. Überteuerte Importpreise, die zu Mehrkosten führten und dadurch die deutsche Wettbewerbsfähigkeit oder das deutsche Preisniveau beeinträchtigten, stellte die BdL in erster Linie im Zusammenhang mit Importen aus den bilateralen Verrechnungsländern fest ${ }^{205}$.

Auch in bezug auf die Existenz von Vorzugsmärkten schätzte sie die Auswirkungen der EZU nicht negativ ein. Während sie warnte, daß eine Förderung des Bilateralismus der deutschen Exportindustrie „leichte, wenn auch kurzfristige Erfolge auf den Märkten der Weichwährungsländer" beschere und so von den schwierigeren Hartwährungsmärkten ablenke, beurteilte sie die durch die EZU geförderten Handelsumlenkungen positiv. In Europa war ihres Erachtens durch

${ }^{203}$ BBK, B 330/2012, Vocke an Erhard, 23.2.1954.

204 BBK, NL Emminger, Dollarlücke, Emminger, Dollarproblem, 1.12.1952; BBK, B 330/2012, Vokke an Erhard, 11.12.1952.

${ }^{205}$ Vgl. BBK, NL Emminger, Konvertibilität/Abbau der Devisenzwangswirtschaft II 1953, o. V. (vermutlich Emminger), Konvertierbarkeit der Währungen von Deutschland her gesehen, o.D. (Mitte 1953). 
die Liberalisierung schon ein „echter marktmäßiger Wettbewerb“ gewährleistet. In der EZU waren nämlich „große Industrieländer" zusammengefaßt, und in diesem Rahmen mußte sich die deutsche Exportindustrie zuerst bewähren ${ }^{206}$. Die EZU würde also die deutsche Wettbewerbsfähigkeit nicht schmälern, sondern langsam und allmählich steigern.

Der Tatsache, daß die EZU eine Abhängigkeit vom europäischen Markt schuf oder stärkte, stand die BdL keineswegs gleichgültig gegenüber. Es war ihr nur allzu deutlich, daß die deutsche Wirtschaft auf die Öffnung der europäischen Märkte nur bedingt zählen konnte. Das hatten die unvollkommen durchgeführte OEEC-Liberalisierung und vor allem die Entliberalisierungsmaßnahmen verschiedener Länder klar gezeigt. Trotzdem sah sie diese Abhängigkeit vom europäischen Markt und die schwankende, nicht fest verankerte europäische Liberalisierung nicht als die wichtigste Problematik an. Der Grund hierfür war in erster Linie, daß die Bundesrepublik ihres Erachtens die Öffnung der europäischen Märkte beeinflussen konnte. Durch eine Good Creditor Policy der Bundesrepublik konnte die europäische Liberalisierung gefördert und weitgehend stabilisiert werden. Im Gegensatz dazu stellte die Bank immer wieder die durch deutsche wirtschaftspolitische Maßnahmen nicht zu beeinflussende Labilität der Dollarbilanz heraus. Permanent war sie beispielsweise um die möglichen Auswirkungen einer amerikanischen Rezession besorgt, denn eine amerikanische Krise würde die deutschen Exporte in die USA zwangsläufig erschweren ${ }^{207}$. Die möglichen negativen Auswirkungen der EZU auf den Außenhandel wurden also durchaus gesehen. Sie wurden jedoch als lösbares und zu beeinflussendes Problem eingeschätzt.

Vor dem Hintergrund einer positiven Zahlungsbilanzentwicklung, ansteigender Währungsreserven, wachsender Überschüsse gegenüber dem EZU-Raum und den bilateralen Handelspartnern mußte die BdL ihre bisherige Haltung zur Konvertibilität überdenken und modifizieren. Sie konnte nicht mehr darauf verweisen, daß die deutsche Zahlungsbilanz und die deutsche Reservenposition der Konvertibilität im Wege stünden. Darüber hinaus mußte sie zu den sich mehr und mehr akzentuierenden Kosten der EZU Stellung nehmen. Im Rahmen dieser Modifizierung schälte sich als ihre Position heraus, daß der Regionalismus und die EZU keinesfalls gefährdet werden sollten. Eine Annäherung an die Konvertibilität lehnte die $B d L$ zwar nicht rundheraus ab, sie ordnete das aber eindeutig der Fortführung der europäischen Kooperation unter. Laut BdL lag diese, vor allem der Erhalt und der Ausbau der europäischen Liberalisierung, im deutschen Interesse, denn sie garantierte der deutschen Wirtschaft einen wenn nicht sicheren, so doch von der deutschen Wirtschaftspolitik zu beeinflussenden Zugang zu Exportmärkten. Sie machte die Bundesrepublik von der amerikanischen Konjunkturentwicklung und Handelspolitik unabhängiger. Demgegenüber sah die

206 Ebenda.

${ }^{207}$ Vgl. BBK, NL Emminger, Dollarlücke, Emminger, Dollarproblem, 1.12.1952. 
BdL die Vorteile, die mit der Konvertibilität verbunden waren -, wie Entgeltung der Überschüsse in Gold und dadurch Ausgleich des traditionellen, „natürlichen" Defizits im Handel mit Übersee durch Überschüsse in Europa; Abbau der Kreditierungsverpflichtungen; Förderung des Kapitalimports -, als unwichtig oder gar als problematisch und unerwünscht an. Auch schätzte sie die mit einer Überschußposition steigenden Kosten des Regionalismus entweder als tragbar ein oder verstand sie nicht als Kosten. Insgesamt wurde so die Konvertibilitätsfrage, bis dato „eine Frage des Könnens, nicht des Wollens“, mehr und mehr zu einer „Frage des Wollens“. Sofern die Liberalisierung eines europäischen Handelspartners durch die Konvertibilität irgendwie gefährdet werden würde, wollte die BdL die Konvertibilität nicht. Sie war in erster Linie an Exportchancen für die deutsche Wirtschaft und an der Liberalisierung der Partner, also an der Konvertibilität der anderen Währungen nach innen, interessiert. Die Konvertibilität der anderen nach außen, z. B. in Form einer Härtung der EZU, rangierte demgegenüber an nachgeordneter Stelle, selbst wenn dadurch ein besserer Ausgleich des Defizits im Handel mit Übersee ermöglicht worden wäre. An der Konvertibilität der DM hingegen war die BdL nur insofern interessiert, als ein Abbau von internen deutschen Restriktionen identisch mit einer Good Creditor Policy war, die ein besseres Funktionieren der EZU erlauben würde. Weitere Ziele, wie billigere Importe, spielten im Zusammenhang mit der EZU keine dominierende Rolle.

\section{Die Bank deutscher Länder und die Umgestaltung} des europäischen und deutschen Währungssystems 1953 bis 1955

Im Sommer 1952 hatten sich die OEEC-Länder im EZU-Direktorium gegen die belgischen Forderungen ausgesprochen und jegliche Härtung der EZU sowie jegliche Annäherung an die Konvertibilität abgelehnt. Trotzdem war damit dieses Thema nicht erledigt. Ganz im Gegenteil: Schon im Herbst 1952 rückte es wieder auf die Tagesordnung. Während in London die Wirtschaftskonferenz der Commonwealth-Länder vorbereitet wurde, breitete sich bei den kontinentalen EZU-Mitgliedern Besorgnis hinsichtlich des britischen Engagements bei der europäischen Währungskooperation aus. Sie befürchteten unilaterale britische Konvertibilitätsmaßnahmen und einen Austritt des Vereinigten Königreiches aus der EZU. Dadurch erhielt das Thema der Konvertibilität eine neue Qualität, und eine erneute Diskussion wurde unabweisbar - zumal das Vereinigte Königreich im Anschluß an die Commonwealth-Konferenz einen Konvertibilitätsplan, den sogenannten Collective Approach towards Convertibility, vorlegte. Deshalb wurden in den folgenden Jahren bi- und multilaterale Gespräche über die britische Vorlage geführt, alternative Konvertibilitätspläne ausgearbeitet, die Reform der EZU wie die Konstruktion eines internationalen Währungssystems debattiert. Hierzu mußten die einzelnen EZU-Mitglieder Stellung beziehen und Ver- 
handlungspositionen ausarbeiten. In der Bundesrepublik war das in erster Linie die Aufgabe des Wirtschaftsministeriums, dem eine Konvertibilitätserklärung obliegen würde, und des Marshallplan-Ministeriums, das für die deutsche Mitarbeit in der OEEC/EZU verantwortlich war. Ferner waren die Handelspolitische Abteilung des Auswärtigen Amtes, federführend für bi- und multilaterale Handelsund Wirtschaftsverträge, das Finanzministerium, zuständig für die Devisenüberwachung und die Auslandsverschuldung, das Landwirtschaftsministerium, beauftragt mit der Agrarpolitik und mit dem Schutz der deutschen Landwirtschaft, und die BdL, Kreditgeberin der EZU, involviert. Schließlich war auch das Bundeskanzleramt beteiligt. Zum einen war die Konvertibilität keineswegs nur ein wirtschaftliches Problem. Vielmehr war sie auch eine politische Frage internationaler Tragweite. Dabei mußte die Bundesrepublik als "besetzter Verbündeter“ die Positionen der Alliierten, vor allem der USA, in Rechnung stellen ${ }^{208}$. Zum zweiten hing mit der Unterzeichnung des EVG- und Deutschlandvertrages die deutsche Souveränität von der Ratifizierung des EVG-Vertrages ab, weshalb den Bemühungen um eine weitergehende europäische Integration ein herausragender Stellenwert zukam. Politik ohne Beachtung dieses vielschichtigen Prozesses war für die Bundesrepublik schlechterdings unmöglich. Die Nachverhandlungen des EVG-Vertrages, weitere daran geknüpfte Projekte wie eine Europäische Politische Gemeinschaft und der Beyen-Plan sowie die Schwierigkeiten dieses Vertragswerkes in der Pariser Nationalversammlung, aber auch die seit Sommer 1954 wieder aktuellen Pläne, die europäische Verteidigung unter Einbezug des Vereinigten Königreiches im Rahmen der NATO bzw. der Westeuropäischen Union (WEU) zu lösen, mußten die deutsche Haltung in allen internationalen Fragen konditionieren ${ }^{209}$.

\section{Internationale Verbandlungen zur Konvertibilität}

1952/53: deutsche Reaktionen auf die britischen Konvertibilitätspläne

Wenn auch das Vereinigte Königreich im Jahre 1952 unter Zahlungsbilanzschwierigkeiten litt, widmete es sich doch der Frage der Konvertibilität. Von einer Ausländer-Konvertibilität erhoffte es sich eine Statusverbesserung des Pfundes, eine Stärkung des Finanzplatzes London und dadurch die Überwindung seiner Zahlungsbilanzprobleme.

${ }^{208}$ Zur amerikanischen Haltung zur Konvertibilität siehe Romero, Interdependence; Boccia, L'amministrazione, S.89ff. Rupieper, Verbündete, bezeichnet die Bundesrepublik als „besetzten Verbündeten". Zu den deutschen Handlungspielräumen vgl. Herbst, Stil.

${ }^{209}$ Für einen Überblick über die Geschichte der europäischen Integration vgl. als eines der letzten Werke internationalen Zuschnittes Trausch (Hrsg.), Integration. Spezifischer auf die Bundesrepublik ausgerichtet sind Bührer/Herbst/Sowade (Hrsg.), Marshallplan; Militärgeschichtliches Forschungsamt (Hrsg.), EVG-Phase; Militärgeschichtliches Forschungsamt (Hrsg.), NatoOption. 
Im Januar 1952 legten das britische Schatzamt und die Bank von England einen ersten Konvertibilitätsplan mit dem Code-Namen ROBOT vor. Zwar lehnte das britische Kabinett diesen Plan sowohl im Februar als auch bei erneuter Vorlage im Juni $\mathrm{ab}^{210}$, doch war damit das Projekt einer Konvertibilitätserklärung nicht erledigt. Schon im Sommer 1952 arbeitete London im Zusammenhang mit der Vorbereitung der Commonwealth-Konferenz einen neuen Konvertibilitätsplan, den Collective Approach towards Convertibility, aus. Wie ROBOT sah auch dieser Plan die Ausländer-Konvertibilität für das Pfund vor. Er nahm eine Änderung der bisherigen Wechselkurspolitik und die Einführung von Handelsbeschränkungen gegenüber nicht-konvertiblen Ländern in Aussicht. Im Unterschied zu ROBOT ging er von gleichzeitigen und abgestimmten Konvertibilitätserklärungen anderer europäischer Länder und einer Unterstützung dieses Schrittes durch die USA aus. Dieses Vorhaben war in mehrfacher Hinsicht mit der EZU unvereinbar. Abgesehen davon, daß eine Wechselkursflexibilität in der EZU vermutlich technisch problematisch war, ließen sich Ausländer-Konvertibilität und eine weitere Mitgliedschaft in der EZU nicht vereinbaren. Darüber hinaus durchbrach die beabsichtigte Einführung von Handelsrestriktionen gegenüber den nicht-konvertiblen Ländern den OEEC-Grundsatz der Nicht-Diskriminierung.

Der Plan ROBOT, der in London hinter verschlossenen Türen diskutiert wurde, blieb in anderen Ländern und in der Bundesrepublik vermutlich unbekannt $^{211}$. Auch über den Collective Approach wußten die OEEC-Mitglieder anfangs nichts Genaues, obwohl sich seit Herbst 1952 Vermutungen und Befürchtungen bezüglich einer britischen Initiative verdichteten ${ }^{212}$. Aber selbst wenn die kontinentalen Länder vorerst hinsichtlich der britischen Pläne im dunkeln tappten, so legte diese Situation nahe, Alternativvorschläge zu entwickeln. Auch mußte das Verhalten im Falle von britischen Maßnahmen diskutiert werden. Unter Umständen war die bisherige Haltung zur EZU zu überdenken, zumal mit diesen britischen Überlegungen offensichtlich wurde, daß die EZU nur eingeschränkt Verbindlichkeit garantieren und als „institutionelle Barriere“ fungieren konnte ${ }^{213}$. Schließlich war der Tatsache Rechnung zu tragen, daß die

${ }^{210} \mathrm{Zu}$ ROBOT vgl. Cairncross, Years, S. $234 \mathrm{ff}$.; Newton, Operation ROBOT; Milward, Motives, S. $261 \mathrm{ff}$.

211 Er hat im Bundesarchiv und im Bundesbankarchiv keine Spuren hinterlassen.

212 Die Vermerke von BdL-Angehörigen zur Commonwealth-Konferenz zeigen, daß man über die britischen Pläne nicht informiert war. Vgl. BBK, B 330/3172, Emminger, Währungspolitische Absichten Großbritanniens, 3.12.1952. Auch die Pariser Gremien tappten im dunkeln; vgl. BBK, B 330/3172, von Mangoldt, Bericht über die 30. Sitzungsperiode des EZU-Direktoriums vom 17.-22.11.1952, 25.11.1952; von Mangoldt an Blücher/Bernard, 27.11.1952.

${ }^{213}$ Mit der EZU hatten sich die OEEC-Mitglieder auf den Abbau von mengenmäßigen Beschränkungen im europäischen Handelsverkehr verpflichtet. Eichengreen, Reconstructing, S. $92 \mathrm{ff}$. bezeichnet die EZU deshalb als eine ,institutional barrier to exit“. Diese Charakterisierung ist zum Teil berechtigt. Allerdings ist Eichengreen zu ergänzen. Es ist festzuhalten, daß die Barrierenfunktion der EZU von Anfang an durchbrochen war. Entliberalisierungen waren aus Zahlungsbilanzgründen gestattet; sie wurden angewandt, und die EZU hatte nur geringe Möglichkeiten, hier einzu- 
EZU durch einen britischen Konvertibilitätsschritt einschneidend verändert würde.

Erstaunlicherweise setzte sich jedoch die BdL im Herbst 1952 mit den britischen Plänen und ihren Konsequenzen aus mehreren Gründen höchstens rudimentär auseinander. Zum ersten rechnete sie nicht ernsthaft mit britischen Maßnahmen ${ }^{214}$. Zum zweiten war für die BdL ohne weitere Diskussion klar, daß im Falle eines britischen Austritts aus der EZU ihre Weiterführung auch ohne das Vereinigte Königreich die einzige Möglichkeit war. Selbst wenn Vocke feststellte, daß ein britischer Austritt die Attraktivität der EZU erheblich schmälern würde, und selbst wenn mit den britischen Projekten deutlich wurde, daß die EZU nicht mehr als eine verläßliche Barriere funktionierte, blieb er bei der Befürwortung der EZU, notfalls in Form einer „Rumpf-EZU“: „Eine solche Beteiligung des Sterlinggebietes liegt auch sehr in unserem Interesse, da ein multilateraler Zahlungsverkehr innerhalb Europas ohne das Sterlinggebiet stark an praktischem Wert verlieren würde und zum Beispiel für Westdeutschland die Möglichkeit entfiele, sein Defizit gegenüber dem Sterling-Raum durch Überschüsse gegenüber kontinentaleuropäischen Ländern abzudecken. [.. . ] Nach meiner Meinung müßte bei Verhandlungen über eine Reform der EZU zunächst auf alle Fälle versucht werden, die Engländer zunächst zum Mitgehen bei einer stärkeren Integration auf monetärem Gebiet zu bewegen. Nur wenn dies scheitern sollte, müßte man sich überlegen, auf welchem Wege man in einem engeren regionalen Kreis Fortschritte erzielen könnte. “215 Zum dritten stand für die BdL wegen der vermutlich flexiblen Wechselkurse und wegen der möglichen Handelsbeschränkungen ein Anschluß an britische Maßnahmen außer Frage. Kurz vor der Commonwealth-Konferenz stellte das BdL-Direktorium entsprechend lapidar fest: „Es herrscht Übereinstimmung darüber, daß deutscherseits feste Kurse beibehalten werden sollten. [...] Das Erreichte (Liberalisierung, Multilateralismus, gemeinsames Handeln) [sollte] nicht aufgegeben, vielmehr tunlich wiederhergestellt werden. “216

Mit dieser abwartenden Haltung befand sich die BdL in guter Gesellschaft. Zwar strebte das Wirtschaftsministerium weiterhin nach der Konvertibilität, wobei vor allem Erhard nach wie vor gegen die EZU Stellung bezog, doch standen sowohl für das Marshallplan-Ministerium als auch für das Auswärtige Amt die Aufrechterhaltung der EZU und ihr reibungsloses Funktionieren im Vorder-

greifen. Seit 1952 wurde die EZU jeden Sommer um ein weiteres Jahr verlängert, wobei Austrittsmöglichkeiten gegeben waren. Insgesamt konnte also die EZU nur für den Zeitraum 1950 bis 1952 - und selbst da nur eingeschränkt - als eine Barriere fungieren.

214 Vgl. BBK, B 330/2055, Direktorium, Protokoll, 4.12.1952. Auch das Wirtschaftsministerium war dieser Meinung. Gleichwohl nahm es die britischen Pläne zum Anlaß, sich intensiv mit der Konvertibilitätsfrage auseinanderzusetzen. Vgl. BAK, B 102/56905, V C 1, Niederschrift über eine Besprechung über Konvertierbarkeit am 22.11.1952, 25.11.1952.

215 BBK, B 330/1313, Vocke an Merton, 15.8.1952.

${ }^{216}$ BBK, B 330/2055, Direktorium, Protokoll, 4.12.1952. 
grund $^{217}$. Auch in den Pariser Gremien herrschte die Meinung vor, die EZU müsse beibehalten werden ${ }^{218}$.

Im weiteren Verlauf mußten sich die Regierung und die BdL allerdings genauer mit den britischen Plänen auseinandersetzen, denn im Januar 1953 kündigte das Vereinigte Königreich informatorische Gespräche mit den USA und mit einzelnen europäischen Regierungen $a^{219}$. Zusätzlich mußte sich die Bank zu der anstehenden EZU-Verlängerung und zu den inzwischen vorgebrachten Ideen einer Reform der EZU eine Meinung bilden. Dabei war zu bedenken, daß sich das Vereinigte Königreich zusammen mit anderen EZU-Schuldnern gegen eine Härtung der EZU ausgesprochen hatte ${ }^{220}$.

$\mathrm{Da}$ die BdL die britischen Pläne einer Ausländer-Konvertibilität ablehnte, sah sie sich vor zwei Optionen gestellt: Entweder konnte sie für eine EZU-Reform plädieren und damit unter Umständen eine Kündigung der britischen Mitgliedschaft befördern; oder sie konnte für eine unveränderte EZU eintreten, hoffend, daß das Vereinigte Königreich in diesem Fall weiterhin teilnehmen würde. Während zu Anfang Vocke der Meinung war, „man [könne] es der OEEC nicht zumuten [. . . ], mit Rücksicht auf die britischen Währungspläne an ihren Reformplänen nicht mehr weiter zu arbeiten “221, setzte sich nach und nach eine kompromißbereite Haltung durch. Da ein britisches Ausscheiden den Wert der EZU mindern würde und da mit dem Auseinanderfallen Europas Handelsbeschränkungen, Diskriminierung gegen konvertible und nicht-konvertible Länder und ein Rückfall in den Bilateralismus verbunden wären, lehnte die Bank eine Reform der EZU ab. Sie wollte das Vereinigte Königreich mit Reformplänen nicht "scheu und mißtrauisch" machen ${ }^{222}$. Auch wollte sie mit einem Verzicht auf Reformen einer Verhärtung der innereuropäischen Fronten entgehen. Vocke befürchtete nämlich, daß der Zusammenhalt der EZU-Mitglieder gefährdet würde, wenn man auf einer Reform bestünde. Dann würden möglicherweise Alternativen zur EZU, wie z.B. eine engere Währungskooperation der sechs EVG-Staaten, in den Blick gerückt. Das aber mußte seines Erachtens vermieden werden, da eine solche Kooperation "sachlich ziemlich unrealistisch" war ${ }^{223}$. Diese Meinung wurde von den Ressorts geteilt. Das Auswärtige Amt betonte zwar, daß die europäischen Integrationsprojekte wie EGKS und EVG einen gemeinsamen Zahlungsmechanismus

217 Vgl. BAK, B 146/480, Protokoll über die Sitzung vom 14.11.1952, 17.11.1952.

218 Vgl. BBK, B 330/3172, von Mangoldt, Bericht über die 30. Sitzungsperiode des EZU-Direktoriums vom 17.-22.11. 1952, 25.11.1952; von Mangoldt an Blücher/Bernard, 27.11.1952. Die Befürwortung der EZU durch die anderen EZU-Mitglieder hielt das BdL-Direktorium in seiner Sitzung am 4.12.1952 fest: „Im übrigen sei nicht die Mehrheit im Verrechnungsmechanismus in erster Linie zu fürchten, sondern die Risiken, die die ,Pfundfreigabe' mit sich bringen." Dieses Risiko hielt sich aber - wie das Direktorium sogleich betonte - in Grenzen, da die deutschen Pfundguthaben gering waren. In: BBK, B 330/2055.

219 Vgl. BAK, B 102/55333, V B 4, Vermerk zum Gespräch Erhard - Trevelyan, 19.1.1953.

220 Vgl. ebenda.

${ }^{221}$ BBK, B 330/2012, Vocke an Erhard, 3. 2.1953.

222 BBK, B 330/2012, Vocke an Erhard, 17.3.1953.

${ }^{223}$ Ebenda. 
notwendig machten. Es wollte aber einen solchen Mechanismus im Kreise der Sechzehn, und nicht im Kreise der Sechs, verwirklicht sehen ${ }^{224}$. Ferner machten Erhard und das Wirtschaftsministerium - trotz aller Befürwortung der Konvertibilität und Kritik an der EZU - angesichts der britischen Konvertibilitätspläne einen Rückzieher. Im Januar 1953 betonte Erhard gegenüber Humphrey Trevelyan, Wirtschaftsberater der britischen Hohen Kommission, daß „er persönlich eine organische Fortentwicklung der EZU und OEEC dringend wünsche und keines-

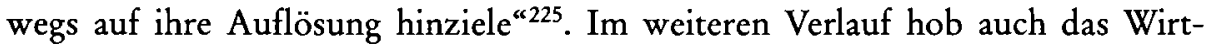
schaftsministerium stets die möglichen negativen Auswirkungen einer Ausländer-Konvertibilität auf den europäischen Handel hervor ${ }^{226}$.

So trug die deutsche Position bezüglich einer EZU-Verlängerung - man solle die Forderung nach Verbesserung und Härtung der EZU zwar „nachdrücklich“ zum Ausdruck bringen, sie aber nicht als Bedingung formulieren; die EZU sei mindestens für ein Jahr zu verlängern - vor allem die Handschrift der BdL und des Auswärtigen Amtes ${ }^{227}$. In den deutsch-britischen Gesprächen über den Collective Approach betonte die deutsche Seite, daß eine Ausländer-Konvertibilität vorerst nicht verfolgt werden sollte, da dabei Handelsbeschränkungen in Kauf genommen würden und der EZU-Raum auseinanderfallen würde. Vielmehr sei die Erhaltung und Ausweitung eines multilateralen Zahlungssystems unabdingbar $^{228}$. In der Konvertibilitätsfrage sei zwar „raschestes Vorgehen“ anzustreben. Angesichts des hohen Anteils des EZU-Raums am deutschen Außenhandel sei aber das „bessere Ordnungssystem insbesondere in einer Reform der EZU zu suchen". Richard A.Butler, der britische Schatzkanzler, war ob dieser Äußerungen erschrocken, denn bisher hatte London die Position und den Einfluß Erhards anders eingeschätzt ${ }^{229}$. Ein Angehöriger der Bank von England faßte die Gespräche

${ }^{224}$ Vgl. BAK, B 146/1843, AA, Abt. II, Londoner Besprechungen über Währungsfragen, 30.4.1953. Vgl. auch BAK, B 146/480, Protokoll über die Sitzung vom 14.11.1952, 17.11.1952. Zur Option einer Währungskooperation der Sechs vgl. BAK, B 102/56 905, Hoffmann-Bagienski, Vermerk, 26.11.1952; BAK, B 102/6428, Albrecht, Zahlungsposition der Montan-Union innerhalb der EZU, 3.12.1952.

${ }^{225}$ BAK, B 102/55333, V B 4, Vermerk zum Gespräch Erhard - Trevelyan, 19.1.1953.

226 Besonders ausgeprägt vertrat die Abteilung $\mathrm{V}$ des Wirtschaftsministeriums diese Meinung. Vgl. BAK, B 102/55345, Abt. V, Allgemeine Auffassung zur Frage der Konvertibilität, 27.4. 1953. Weniger deutlich, aber doch insgesamt skeptisch zu den britischen Plänen war aber auch die Grundsatzabteilung I.Vgl. BAK, B 102/12651, I A 1, Vermerk, 5.5.1953. Obwohl sich das Wirtschaftsministerium gegen die britische Initiative aussprach, war Vocke von der Standfestigkeit des Wirtschaftsministeriums nicht überzeugt. Er verlangte eine Diskussion im Kabinett. Bei Anwesenheit von Bundeskanzler und Außenminister Adenauer, Landwirtschafts-, Marshallplan- und Finanzminister hätte diese mit großer Wahrscheinlichkeit zu einer Warnung vor übereilten Schritten geführt. Vgl. BAK, B 146/1843, Vogel, Vermerk, 27.4.1953; BAK, NL Hallstein, Hallstein, Notizen über eine Sitzung beim Vizekanzler Blücher, 27.4.1953.

${ }^{227} \mathrm{Vgl}$. BAK, B 146/481, BMM, Ergebnisbericht einer interministeriellen Besprechung über Verlängerung und Verbesserung der EZU, 17.3.1953.

${ }^{228}$ Vgl. BAK, B 146/1843, BMWi, Fragebogen zur internen Verwendung, 7.5.1953.

229 Vgl. BBK, B 330/3172, Emminger, Zusammenfassender Bericht, 22.5. 1953; BBK, B 330/2056, Direktorium, Protokoll, 15.5.1953. Vgl. auch die Protokolle der deutsch-britischen Besprechungen in PRO, T 236/3519. 
folgendermaßen zusammen: "What did show up very clearly in the German talks was that Erhard's (Minister of Economy) frequent pronouncements on the aims of Germany being convertible were by no means reflected in officials' minds. Although the latter liked the idea of convertibility they were very anxious about its effects on their European trade. ${ }^{\text {"230 }}$

Wenngleich das Pressekommuniqué der deutsch-britischen Gespräche auf das erzielte Einvernehmen abstellte, war deutlich geworden, daß die Bundesrepublik nicht bereit war, die Kosten der Konvertibilität zu tragen und das Vereinigte Königreich in einem Konvertibilitätsschritt zu unterstützen. Auch wenn es die Konvertibilität nicht prinzipiell ablehnte, schob sie sie vorerst auf die längere Bank. Donald Macdonald, nun Angehöriger der Bank von England, stellte heraus: „Whilst it does not seem that we can count at this stage on active German support for our plans, it would not be right to assume their hostility. They showed genuine interest and their approach was not destructive. Nevertheless it cannot be said that they were enthusiastic. ${ }^{\text {2231 }}$

\section{3/54: der Weg zur Konsolidierung}

Die Tatsache, daß die deutsche Regierung in den Gesprächen mit dem Vereinigten Königreich eine EZU-freundliche Haltung eingenommen hatte, bedeutete keineswegs, daß sich eine definitive deutsche Position herausgeschält hatte. Schon bald darauf fanden Erhard und die Abteilung I des Wirtschaftsministeriums (Grundsatzabteilung) zu ihrer negativen Haltung zur EZU zurück. Gestützt auf ein Gutachten von Professor Friedrich Lutz ${ }^{232}$, argumentierten sie, die Möglichkeiten der EZU seien erschöpft. Eine schrittweise Weiterentwicklung der EZU sei irrealistisch und zu langwierig ${ }^{233}$. Die EZU würde die deutsche Expansion behindern. Sie wäre mit „unfreiwilligem oder unbewußtem Kapitalexport" verbunden und würde über kurz oder lang künstliche Beschränkungen des lebensnotwendigen deutschen Exportes erfordern. Die deutschen Überschüsse zum EZU-Raum seien strukturell, so daß man ihnen nicht mit ad-hocMaßnahmen begegnen könne. Eine hundertprozentige Liberalisierung müßte „zu einer gewissen Verzerrung der Verhältnisse in Europa" führen. Deshalb seien jetzt „neue Formen“, namentlich die Konvertibilität, herzustellen, wobei die

${ }^{230} \mathrm{BoE}, \mathrm{OV}$ 34/39, Watson, Bilaterals with Germany and Belgium, 21.5.1953.

231 BoE, OV 34/39, Macdonald, Economic discussions with Germany, 14.5.1953.

${ }^{232}$ Das Wirtschaftsministerium hatte Lutz mit einem Gutachten zur Konvertibilität beauftragt. Es wurde leicht überarbeitet als Lutz, Problem, veröffentlicht.

${ }^{233}$ Für eine anschauliche Darstellung der in der Grundsatzabteilung des Wirtschaftsministeriums vertretenen Meinungen vgl. BAK, B 102/12655, Möller, Vermerk, 29.7.1953. Die wichtigsten grundsätzlichen Ausarbeitungen sind: BAK, B 102/12651, I A 1, Fragen des Zusammenlebens konvertierbarer und nicht-konvertierbarer Währungen, 26.6.1953; I A 1, Arbeitsunterlage zur Vorbereitung einer Stellungnahme des BMWi, 27.8.1953; I A 1, Probleme der Konvertibilität, 21.12.1953; BAK, B 102/55 345, I A 1, Vermerk für Herrn Minister, 14.10.1953; BAK, B 102/11137, I A 1, Vorbereitung der Ministerratstagung am 29./30.10.1953. Vgl. auch BAK, B 102/55333, Müller-Armack, Diskussionsbeitrag zum Thema Exportförderung, 20.6.1953; BAK, B 146/483, I A 5, Gespräch Erhard - Marjolin, 16.10.1953. Die folgenden Zitate sind diesen Dokumenten entnommen. 
Einführung flexibler Wechselkurse notwendig sei. Die an der Konvertibilität wirklich interessierten Länder - das Vereinigte Königreich, Belgien, Holland, Schweiz und vielleicht Italien - müßten sich zusammenschließen und außerhalb der EZU vorgehen. Die Probleme, die sich dabei ergeben würden, ließ die Grundsatzabteilung weitgehend unbeachtet oder negierte sie gar. Zu den Schwierigkeiten eines Zusammenlebens konvertibler und nicht-konvertibler Länder bemerkte sie nur etwas blauäugig, die nicht-konvertiblen Länder sollten die konvertiblen „wohlwollend unterstützen“. Sie sollten vor allem ihre Liberalisierung aufrechterhalten. Darüber hinaus hielt sie fest, daß das Problem eines Auseinanderfallens nicht existiere, denn ein isoliertes Vorgehen würde "vermutlich“ andere Länder dazu veranlassen, sich anzuschließen. Mit Blick auf das Auswärtige Amt stellte die Grundsatzabteilung ohne auf konkrete Schwierigkeiten einzugehen fest, daß die Konvertibilität die europäischen Integrationsbemühungen nicht erschwere, sondern eine Voraussetzung für sie sei.

Innerhalb Deutschlands kam die Opposition zu Erhards erneuten Vorstößen von verschiedenen Seiten. Zum einen war das Wirtschaftsministerium keineswegs einer Meinung. Laut Abteilung V (Außenwirtschaft) war die Abteilung I (Grundsatzabteilung) in ihrer Beurteilung der EZU „zu einseitig“. Sie stellte heraus, das Risiko einer Diskriminierung sei in der Konvertibilitätsfrage entscheidend und bisher nicht gelöst. Die Voraussetzungen, ausreichende europäische Reserven sowie eine Good Creditor Policy der USA, müßten erst noch geschaffen werden ${ }^{234}$. Zum zweiten opponierte die BdL die Erhardsche Befürwortung der Konvertibilität. In gewohnter Schärfe stellte sie fest, die EZU sei in keiner Weise festgelaufen. Deshalb müsse „unsere positive Stellung zur EZU“ betont werden ${ }^{235}$. Unterstützt wurde die BdL hierin von der Bundesvertretung in Paris und von Adenauer, der Erhard aus politischen Gründen zur Ordnung rief und sogar mittels Weisungen eingriff ${ }^{236}$. Zusätzlich zu dieser prinzipiellen Befürwortung der EZU hielt Vocke weiterhin die von Erhard bei Konvertibilität vorgesehene Wechselkursflexibilität für „schädlich, ja unerträglich“. Diese würde nur „Chaos“ stiften und sei „voller Ungewißheit und Risiken “237. Unterstützt wur-

${ }^{234}$ BAK, B 102/56 905, V D 1, Besprechung vom 2.9.1953, 3.9.1953. Vgl. BAK, B 102/55 345, Stedtfeldt, Wege zur Konvertibilität, 13.10.1953; BAK, B 146/482, II/5 (Fünfgelt), Vermerk 12.12.1953.

${ }^{235}$ BBK, B 330/2033, Vocke an von Mangoldt, 5.10.1953. Vgl. auch BAK, B 146/483, Schleiminger, Ergebnisbericht einer Besprechung über Fragen der Konvertierbarkeit, 11.8.1953; BBK, NL Emminger, Konvertibilität/Abbau der Devisenzwangswirtschaft II 1953, BdL, Vermerk über eine zweite Ressortbesprechung mit Herrn Professor Lutz, 14. 8. 1953.

236 Vgl. Kabinettsprotokolle VI (1953), 7. Kabinettssitzung am 10.11.1953, S. 510; LESt, I 1)2, Erhard an Adenauer, 7.12.1953. Für die Position der Bundesvertretung vgl. BAK, B 102/12655, Möller, Vermerk, 29.7.1953.

${ }^{237}$ BBK, NL Emminger, Flexible Wechselkurse, Vocke, Vermerk zu Frère-Vorschlag, 6.10.1953; BBK, B 330/3379, Vocke, Vermerk, 21.10.1953. Emminger und Wolf fertigten fundiertere und nicht so emotionale Vermerke zu dieser Problematik an. Dabei kamen auch sie zu einem negativen Urteil. Vgl. BBK, NL Emminger, Flexible Wechselkurse, Emminger, Frère-Vorschlag einer 
de Vocke hierin von dem renommierten Wirtschaftswissenschaftler Wilhelm Röpke und angeblich allen Notenbanken ${ }^{238}$.

Aber auch außerhalb der Bundesrepublik lief Erhard mit seinen Konvertibilitätsplänen in schweres Wasser, und das stärkte die innerdeutsche Opposition. Abgesehen von dem Widerstand innerhalb der EZU nahmen die USA eine abwartende Haltung ein. Sie hoben hervor, daß eine amerikanische Hilfestellung zugunsten einer Konvertibilitätsaktion und entscheidende Maßnahmen auf dem Gebiete der amerikanischen Handelspolitik ,auf keinen Fall“ vor Ende 1954 zu erwarten seien. Die USA wollten zuerst den Bericht des Randall-Ausschusses, der die internationale Wirtschaftspolitik der USA überprüfen sollte, und die Kongreßwahlen im November 1954 abwarten $^{239}$. An dieser abwartenden Haltung Amerikas konnte auch Erhards Reise in die USA, auf der er für die Erklärung der Konvertibilität warb, nichts ändern. Selbst das amerikanische Schatzamt, das der Konvertibilität positiv gegenüberstand, plädierte im Anschluß an die Gespräche mit Erhard für ein langsameres Vorgehen: „Dr. Erhard was vigorous and outspoken. [...] His intentions are in the right direction, but whether Germany and other ,hard' countries in Europe are ready for a vigorous move towards convertibility requires careful study. “240

Vor diesem Hintergrund konnte Emminger anläßlich einer neuerlichen, vom Wirtschaftsministerium initiierten Konvertibilitätsbesprechung, für die das Ministerium verbindliche Stellungnahmen forderte, Anfang 1954 feststellen, daß Eile unangebracht sei: „Ich bin der Ansicht, daß es ziemlich sinnlos wäre, zu allen in dem Entwurf des Bundeswirtschaftsministeriums angeschnittenen theoretischen Fragen im gegenwärtigen Stadium verbindliche Äußerungen abzugeben. Es scheint mir absolut kein Bedürfnis dafür vorzuliegen, hic et nunc und ohne jeden konkreten Anlaß und $Z$ wang bindende Beschlüsse [. . . zu fassen. ${ }^{\text {"241 }}$

Da weder der britische Collective Approach noch Erhards Ansichten die notwendige Zustimmung fanden und auch eine schrittweise Härtung der EZU abgelehnt wurde, zeichnete sich im Herbst 1953 ein Stillstand bei den europäischen

größeren Schwankungsbreite, 5.10.1953; Wolf, Vermerk, Zur Frage der fluktuierenden Wechselkurse, o.D. (Oktober 1953); Emminger an Donner, 5.10.1953.

${ }^{238}$ Vgl. Röpke, Wege; LESt, I 4)59, Röpke an Erhard, 27.10.1953. Vocke behauptete in einem Vermerk - vgl. BBK, B 330/3379, Vocke, Vermerk über BIZ-Sitzung, 21.10.1953-, daß „keine einzige Notenbank" flexible Kurse befürworte. Wenn man bedenkt, daß ROBOT von der Bank von England und der Frère-Vorschlag von der Banque Nationale de Belgique vorgelegt wurden, erscheint das übertrieben.

${ }^{239}$ Vgl. BAK, B 146/482, von Mangoldt an Blücher, 23.9.1953.

${ }^{240}$ NARA, RG 56/28, EUR/3/11, Hebbard, Meeting with Dr. Erhard, 2.12.1953. Vgl, auch die Protokolle der Gespräche in NARA, RG 59 862A.00/11-2353 bis 862A.00/11-2553. Vgl. auch die sicherlich nicht unparteiische Beschreibung von Hall-Patch, britischer Exekutiv-Direktor beim IWF, der den Erhard-Besuch als „particular comedy“ bezeichnete und die amerikanische Irritation ob Erhards Wortschwälle wie auch die amerikanische Begeisterung angesichts der nicht erfolgten Bitte um Kredithilfe notiert. In: PRO, T 236/3519, Hall-Patch an Rowan, 12.12.1953.

${ }^{241}$ BBK, NL Emminger, Vermerke zur Konvertibilität, Emminger, Vermerk, 15.1.1954. 
Konvertibilitätsdiskussionen ab. Erst Anfang des Jahres 1954 wurde dieser aus zwei Gründen überwunden. Erstens schloß Ende Januar 1954 die vom amerikanischen Präsidenten Dwight D. Eisenhower eingesetzte Commission on Foreign Economic Policy ihre Überprüfung der amerikanischen Außenwirtschaftspolitik ab. Dabei sprach sie sich für Konvertibilität und ein weltweites multilaterales Handels- und Zahlungssystem aus. Gleichzeitig aber plädierte sie für ein vorsichtiges Vorgehen. Sie stellte heraus, „daß sie (die USA, M.D.) keinerlei Maßnahmen unterstützen sollten, welche zur Beseitigung der EZU führen könnten, solange nicht etwas Besseres an ihre Stelle gesetzt wird “242. Zweitens wurde deutlich, daß die EZU-Schuldner den Forderungen der EZU-Gläubiger nach einer Reform entgegenkommen mußten, sofern sie am Funktionieren der EZU interessiert waren. $\mathrm{Da}$ die im EZU-System vorhandenen Kredite fast vollständig ausgeschöpft waren $^{243}$, gleichzeitig eine Entschärfung der extremen Gläubigerpositionen unmöglich gewesen war und mit Aufnahme der Liberalisierung zum Dollarraum in immer weitere Ferne zu rücken drohte, lag es im Interesse einiger Schuldner, sich dem stetigen und letzthin sehr erhöhten Druck der Gläubiger zu beugen ${ }^{244}$. Der hierfür notwendige Handlungsspielraum war dabei in vielen Fällen insofern gegeben, als zumindestens einzelne Länder im Verlauf des Jahres 1953 konvertible Währungsreserven akkumuliert hatten ${ }^{245}$.

$\mathrm{Da}$ auch Italien einerseits im Jahre 1953 seine konvertiblen Reserven um 140 Mio. Dollar erhöht hatte, andererseits aber ein hohes kumulatives EZU-Defizit aufwies, schlug es Anfang 1954 einigen seiner Gläubiger vor, einen Teil seiner EZU-Schulden zurückzuzahlen, einen Teil zu konsolidieren ${ }^{246}$. Im April 1954 formulierte das Vereinigte Königreich einen ähnlichen Vorschlag: Es bot seinen Gläubigern eine teilweise Konsolidierung und teilweise Rückzahlung - bzw. im

${ }^{242}$ Vgl. Commission on Foreign Economic Policy, Report. Auszugsweise übersetzt in BBK, B 330/ 75.

${ }^{243}$ Im EZU-System standen noch Kredite in Höhe von 196 Mio. RE zur Verfügung. Allerdings täuscht diese Zahl insofern, als sich beispielsweise Dänemark angesichts seiner Reservensituation die bei Inanspruchsnahme seiner Kreditlinie zu leistenden Goldzahlungen nicht erlauben konnte. Cahan vom Trade and Finance Directorate der OEEC meinte deshalb, das Ende der EZU sei abzusehen. Vgl. HAEC, OEEC-TRA 337, Cahan an Marjolin, 9.3.1954. Auch Ellis-Rees, britischer Vertreter im EZU-Direktorium, sprach vom nahenden Kollaps der EZU. Vgl. HAEC, OEECTRA 337, UK Delegate, Statement to the Council, 2.4.1954.

${ }^{244}$ Vgl. HAEC, OEEC-TRA 337, Cahan an Colonna, 19.1.1954. Vgl. auch BoE, OV 46/16, Bolton, Conversations at Basle, 12.1.1954; BoE, G 1/188, Bolton, Conversations at Basle, 10.2.1954.

${ }^{245}$ Im Verlauf des Jahres 1953 wuchsen die konvertiblen Reserven wie folgt an (Mio. \$): Österreich +95 , Belgisch-Luxemburgische Wirtschaftsunion +63 , Dänemark +26 , Frankreich +85 , Bundesrepublik Deutschland +534 , Griechenland +55 , Italien +140 , Niederlande +240 , Norwegen +11 , Portugal +95 , Schweden +60 , Schweiz +80 , Türkei +6 , Vereinigtes Königreich +691 . Vgl. BIS, 24 th Annual Report (1953/54), S. 157.

246 Der italienische Vorschlag führte zu heftigen Auseinandersetzungen innerhalb des Zentralbanksystems. Während Emminger und der Zentralbankrat den italienischen Vorschlag sorgfältig prüfen wollten, lehnte Vocke das italienische Ansinnen ab und schrieb dementsprechend an von Mangoldt. Vgl. BBK, B 330/74, ZBR, Protokoll, 20.1.1954; BBK, B 330/1313, Vocke an von Mangoldt, 27.1.1954; Emminger, Vermerk 28.1.1954. Am 3.2.1954 wurde das Thema deshalb nochmals im Zentralbankrat aufgenommen. Vgl. BBK, B 330/75, ZBR, Stenogramm, 3.2.1954. 
deutschen Fall eine Aufrechnung mit den deutschen Regierungsschulden - an. Als Gegenleistung verlangte es von der Bundesrepublik eine Good Creditor Policy und die Bereitstellung neuer EZU-Kredite ${ }^{247}$.

Wenn auch der informelle Gläubigerklub der EZU, an dem der deutsche EZUVertreter inzwischen teilnahm, seit November 1953 Rückzahlungen der ausstehenden Kredite befürwortete, traf der britische Vorschlag in der Bundesrepublik auf einige Skepsis. Das Ministerium für wirtschaftliche Zusammenarbeit sah diesen Vorschlag nicht als eine Härtung der EZU an ${ }^{248}$. Bei den ersten Sondierungen mit den Briten führte sein Vertreter aus, die EZU würde dadurch aufgeweicht, so daß eine solche Konsolidierung eher ein Rückschritt sei. Auch die BdL lehnte den britischen Vorschlag ab. Sie wies darauf hin, daß die deutschen EZU-Guthaben relativ liquide Währungsreserven seien und als solche nicht gegen Regierungsschulden eingetauscht werden könnten ${ }^{249}$. Das Problem der Währungsreserven war aber nicht der entscheidende Grund für die ablehnende Haltung der BdL. Nach Gesprächen mit Vocke vermutete Cameron Cobbold, Gouverneur der Bank von England, vielmehr, daß die BdL dem Vereinigten Königreich durch ihre Zustimmung zur Konsolidierung keine freiere Hand für eine Konvertibilitätserklärung geben wollte: „I have had a bit of talk with Dr. Vocke about EPU. [...] His line is that consolidation now is a mistake because nobody knows what will happen in next year. I guess, like the Dutch, he is afraid a settlement with us this year would leave us freer to bully them about commercial policy next year. He has been making a good deal of the Central Bank difficulty about funding - but I suspect that this is mainly producing an argument in favour of leaving settlement until end of EPU. I think he would find no difficulty in coping with that point if the governments were to agree some set-off arrangement. “250 Damit traf Cobbold den Kern der Sache. Im Zentralbankrat stellte Vocke als wichtigsten Punkt heraus, daß wir „kein Interesse an einer vorzeitigen Abwicklung der EZU haben. Umgekehrt ist es mit England. Es will frei konvertierbar sein. Die Engländer müssen bei einer Liquidation zahlen. Ist das auch noch beseitigt, können sie sagen: Wir sind völlig frei. ${ }^{\text {"251 }}$

Die negative deutsche Haltung verflüchtigte sich aber schon bald. Ende April befürwortete sowohl Erhard als auch Hermann J.Abs, enger Wirtschaftsberater Adenauers, den britischen Konsolidierungsvorschlag. Sie stellten fest, daß Konsolidierungen ein Weg zur Konvertibilität seien ${ }^{252}$. Erhard, der die EZU ablehn-

\footnotetext{
247 Vgl. PRO, T 232/362, Rowan, Note EPU, Germany etc., 27.3.1954.

248 Vgl. BAK, B 102/56 905, II/5, Vermerk zur Reform der EZU, 12.2.1954.

249 Vgl. PRO, FO 371/111 199, UK Delegation on Foreign Office, 21.4. 1954; UK Delegation, Record of a discussion with the German delegation, 21.4.1954. Diese Schwierigkeiten der BdL waren von der Bank von England vorausgesehen worden. Vgl. PRO, T 232/362, Bank of England, Note, 26.3.1954.

250 BoE, OV 34/39, Cobbold, Note, 5.4.1954.

251 BBK, B 330/77, ZBR, Stenogramm, 28.4.1954. Vgl. auch PRO, FO 371/111 199, UK Delegation, Record of a discussion with the German delegation, 21.4.1954

${ }^{252} \mathrm{Vgl}$. PRO, FO 371/111 199, Mason an Foreign Office, 27.4.1954.
} 
te und dementsprechend die Befürchtungen Vockes nicht teilte, verkündigte sogar, die Bundesrepublik würde die Konvertibilität der DM „Fünf Minuten nach England“ erklären ${ }^{253}$. Wenig später rückten auch Vocke und das BdL-Direktorium von ihrer bisherigen Position ab und stellten sich einer Konsolidierung nicht mehr in den Weg - auch wenn der Zentralbankrat dies nicht eindeutig abgesegnet hatte ${ }^{254}$. Nachdem auf dieser Grundlage ein deutsch-britisches Übereinkommen erzielt war, war der Weg für weitere bilaterale Konsolidierungen frei. Im Sommer 1954 wurden als Ergebnis weiterer Verhandlungen EZU-Kredite in Höhe von insgesamt 858 Mio. RE bilateral konsolidiert. Dabei fielen Goldzahlungen in Höhe von 224 Mio. Dollar an. Zusätzlich dazu zahlte die EZU 130 Mio. Dollar aus ihren Reserven an die Gläubiger. Auch wurden neue Kreditierungsverpflichtungen in Höhe von $1 \mathrm{Mrd}$. RE vereinbart, wobei die EZU bei leichter Erhöhung des Gold-Kredit-Verhältnisses gleichzeitig für ein weiteres, möglicherweise letztes Jahr verlängert wurde ${ }^{255}$.

Mit der deutschen Zustimmung zur Konsolidierung war eine neue Politiklinie eingeschlagen worden. Anstatt auf einer schrittweisen Härtung der EZU zu bestehen, stimmten die Bundesregierung und die BdL einer gewissen Aufweichung der EZU zu ${ }^{256}$. Gleichzeitig akzeptierte die BdL, daß die EZU höchstwahrscheinlich bald - nämlich im Sommer 1955 - zugunsten einer Ausländer-Konvertibilität beendet werden würde. Während die Zustimmung Erhards hierzu nicht

${ }^{253}$ BBK, B 330/3171, V A 8, Aufzeichnung über die Besprechungen mit Schatzkanzler Butler am 7./ 8.5.1954, 11.5.1954.

254 Der Zentralbankrat diskutierte die britischen Vorschläge mehrmals, nämlich am 28.4., am 19.5. und am 2.6.1954. Zum Diskussionsverlauf vgl. die Protokolle und Stenogramme der Zentralbankratsitzungen in BBK, B 330/77. Am 2.6.1954 diskutierte er den zu erreichenden Zinssatz, die Laufzeit und weitere technische Einzelheiten. Danach ermächtigte er das Direktorium zu Verhandlungen. Als die Verhandlungen kurz vor Abschluß standen und als Erhard herausstellte, die Bundesrepublik würde die Konvertibilität fünf Minuten nach dem Vereinigten Königreich erklären, zeigte sich, daß der Zentralbankrat diese Wendung der Verhandlungen nicht intendiert hatte. In der Sitzung am 16.6.1954 griff er die schon fortgeschrittenen und „wohl kaum noch revidierbaren Verhandlungen“ nochmals grundsätzlich auf. Pfleiderer stellte hierzu fest: „Von Herrn Wolf haben wir gehört, daß die BdL eine Linie hat, nämlich die Linie: keinesfalls irgendwelche bilateralen und multilateralen Zahlungsabkommen mehr, sondern Konvertibilität, d.h. freier Dollarzahlungsverkehr auf der ganzen Linie. Mir ist das bis jetzt nicht bewußt geworden, daß die BdL diese Linie hatte, obwohl ich eigentlich der Meinung bin, daß diese Linie der BdL eine Sache des Zentralbankrates ist. Herr Vocke und der Zentralbankrat haben diese Linie sicherlich nicht gemacht. “ BBK, B 330/78, ZBR, Stenogramm, 16.6.1954.

255 Für die Einzelheiten vgl. EPU, 4 th Annual Report (1953/54), S. $95 \mathrm{ff}$.

$256 \mathrm{Vgl}$. Kaplan/Schleiminger, EPU, S.202ff., die die EZU-Reform von 1954 als eine erste Härtung der EZU, als einen ersten Sieg des sogenannten Institutional Approach, feiern. Mit dem Wissen, daß im Jahre 1955 eine zweite Härtung erfolgte, stellen sie fest: „The 1954 package set the stage for agreement on further hardening." Das stimmt aber nur bedingt. Zum einen ist umstritten, ob die EZU wirklich härter wurde. Die neuen Kreditierungsverpflichtungen bedeuteten eine Aufweichung. Zum zweiten war die Erhöhung des Gold-Kredit-Verhältnisses für die Gläubiger insofern irrelevant, als sie zumeist ihre Quoten schon überschritten hatten, in den Rallongen aber gewöhnlich ein Gold-Kredit-Verhältnis von $50 \mathrm{zu} 50$ gewährt wurde. Ferner ist festzuhalten, daß das Abkommen auf der Idee beruhte, dies sei wahrscheinlich die letzte Verlängerung der EZU. Demnach wäre die EZU-Reform des Sommers 1954 als erster und einziger Schritt des sogenannten Institutional Approach konzipiert gewesen. 
verwundert, bedeutete sie für die BdL - trotz des letztlich rauheren Tons gegenüber der EZU ${ }^{257}$ - einen erheblichen Meinungswandel binnen kürzester Zeit. Dieser muß erklärt und analysiert werden.

Eine mögliche Erklärung könnte darin liegen, daß das Gold-Kredit-Verhältnis erhöht wurde. Das war jedoch für die BdL nicht entscheidend. Ganz im Gegenteil: Die Erhöhung des Goldanteils von 40 auf 50 Prozent stieß innerhalb der BdL auf große Skepsis. Emminger bezeichnete sie als „eine rein optische Härtung", an der die BdL kein Interesse habe, gegen die sogar Bedenken bestünden ${ }^{258}$. Eher kann die Zustimmung der BdL deshalb mit der Goldzahlung erklärt werden, die mit der Konsolidierung verbunden war. Wenn auch Max Grasmann, Präsident der Landeszentralbank Bayern, festhielt, daß das eine "primitive Überlegung" sei, wies Bernard auf diese "glückliche Lösung" hin und faßte als vorherrschende Meinung zusammen: „Wir kommen auch gar nicht so schlecht weg dabei, denn wir kriegen 170 Mio. Dollar sofort gezahlt. “259 Ein weiterer Teil der Erklärung ist darin zu finden, daß man während der Verhandlungen eine Lösung für die Frage der Währungsreserven gefunden hatte. Die Abkommen sahen vor, die konsolidierten Beträge bis zur effektiven Rückzahlung in der EZU zu behalten. So konnte im Falle von Defiziten auf diese Beträge, die damit weiterhin liquide Währungsreserven waren, zurückgegriffen werden. Der wichtigere Teil der Erklärung ist aber darin zu suchen, daß wirtschaftliche wie politische Gründe für ein Entgegenkommen sprachen.

Als wirtschaftlichen Grund führte die BdL an, daß sich die Währungsreserven der EZU-Länder erhöht hätten, so daß die Dollarknappheit nicht mehr existiere, also die mit der Konsolidierung in unmittelbare Nähe gerückte Konvertibilität die europäische Liberalisierung nicht gefährden würde ${ }^{260}$. Vor diesem Hintergrund bewertete Wolf die Teilnahme des Sterlinggebietes an der EZU als den „eigentlichen Reiz der Verrechnung über die EZU“ und bezeichnete die Option einer Rumpf-EZU als nicht erstrebenswert ${ }^{261}$. Auch Emminger hob hervor, die einzige Option für die Bundesrepublik sei, einer britischen Initiative zu folgen, denn „England [habe] aufgrund seiner allgemeinen Position auf jeden Fall das

257 Vgl. BBK, B 330/2033, Vocke an von Mangoldt, 31.3.1954; BBK, B 330/1313, Vocke an Blücher, 12.3.1954. Gleichzeitig gab es aber auch weiterhin ausgesprochen positive Stellungnahmen zur EZU. Vgl. BBK, B 330/2012, Vocke an Erhard, 23.2.1954; BBK, B 330/77, ZBR, Stenogramm, 28.4.1954.

${ }^{258}$ BBK, B 330/3171, Emminger, Grundzüge der Vereinbarungen für die Verlängerung der EZU, 28.6. 1954; BBK, B 330/77, ZBR, Stenogramm, 2.6.1954; BBK, B 330/1310, Emminger, Sitzung des kleinen Wirtschaftskabinetts, 4.6.1954. Für die Bundesrepublik war die Erhöhung des Goldanteils nicht mit erhöhten Goldzahlungen verbunden, denn sie glich ihre Überschüsse bereits in Rallongen aus.

259 BBK, B 330/78, ZBR, Stenogramm, 16.6.1954. Diese Angabe ist nicht ganz korrekt. Die Zahlung belief sich auf 146,96 Mio. \$.

260 Vgl. BBK, NL Emminger, Vermerke zur Konvertibilität, Emminger, Bemerkungen zu den Vorschlägen für einen Europäischen Fonds, 26. 8.1954.

${ }^{261} \mathrm{BBK}, \mathrm{B} 330 / 78, \mathrm{ZBR}$, Stenogramm, 16.6.1954. 
Schicksal der EZU in der Hand [...] und [...] einmal vor die Tatsache einer englischen Konvertibilität und der EZU-Beendigung gestellt, [werden wir] sowieso nur zwei Wege haben [. . . ]: entweder Konvertibilität oder Bilateralismus. Natürlich werde man sich für ersteren entscheiden. "262 Hinzu kam, daß Wolf und Emminger keineswegs überzeugt waren, die Auflösung der EZU zum Sommer 1955 wäre zwangsläufig. Sie waren vielmehr der Ansicht, daß das Abkommen das Weiterbestehen der EZU für ein weiteres Jahr, möglicherweise noch darüber hinaus, gesichert habe ${ }^{263}$. Deshalb könne die Bundesregierung, die BdL und Europa nun die Zeit für „die notwendigen Vorbereitungen für einen kollektiven und mit gewissen Sicherungen versehenen Übergang zur Konvertierbarkeit" nutzen. Demgegenüber „[hätte] ein kompromißloses Festhalten an ihnen (den ursprünglichen Forderungen der Gläubiger, M.D.) nach der Sachlage mit Sicherheit zu einer Sprengung der EZU geführt ${ }^{\text {«264. }}$.

Die wirtschaftlichen Gründe können jedoch nicht das alleinige Motiv für das Entgegenkommen gewesen sein. Da sich beispielsweise die europäische Reservensituation langsam und allmählich verbessert hatte, kann sie den plötzlichen Meinungsumschwung der BdL nicht zufriedenstellend erklären. Tatsächlich scheinen letztendlich politische Überlegungen den Ausschlag gegeben zu haben. Das legen zumindest Andeutungen Vockes und Bernards nahe. Während sich das deutsch-französische Verhältnis über die französischen Schwierigkeiten einer EVG-Ratifizierung abgekühlt hatte, Adenauer vom Scheitern der deutschfranzösischen Annäherung und einem Entgegenkommen an das Vereinigte Königreich sprach, und während innerhalb der BdL die „Vorläufigkeit unserer ganzen Gesetzgebung und unseres Verhältnisses zur Regierung wie auch die Vorläufigkeit der Regelung der Herrschaft über die Devisen" herausgestellt wurden, räumte Bernard in der Sitzung des Zentralbankrates am 16.Juni 1954 ein: „Wir [machen] sehr viel Konzessionen [...], offenbar mehr, als es vielleicht unbedingt nötig wäre, und [...] dabei [spielt] im Hintergrund - mindestens bei der Regierung - ein Wunsch doch wohl eine Rolle [...]: England doch sehr freundlich entgegenzukommen, vielleicht aus Gründen, die in der reinen Außenpolitik liegen. [...] Die sehr generelle allgemeine Beurteilung, daß wir hier nicht unbedingt das allerbeste für uns herausholen, sondern tatsächlich einige Konzessionen machen, die vielleicht von allgemeinen politischen Erwägungen diktiert sind, teile ich auch. ${ }^{\text {"265 }}$

Da die BdL dem Vereinigten Königreich und Adenauer aus außen- und innenpolitischen Gründen entgegenkommen wollte, ging sie in den Konsolidierungs-

${ }^{262}$ Wiedergabe der Meinung Emmingers durch von der Lippe, vgl. BBK, B 330/2032, von der Lippe an Vocke, 1.7.1954.

${ }^{263}$ Vgl. BBK, B 330/78, ZBR, Stenogramm, 16.6. 1954; BBK, B 330/3171, Emminger, Grundzüge der Vereinbarungen für die Verlängenung der EZU, 28.6.1954.

264 BBK, B 330/78, ZBR, Protokoll, 30.6.1954.

${ }^{265} \mathrm{BBK}, \mathrm{B} 330 / 77$ und 78, ZBR, Stenogramm, 2.6.1954 und 16.6.1954. Vgl. auch BoE, OV 34/67, Bolton, German trends, Basle conversations, 8./10.5.1954, 12.5.1954. 
verhandlungen Kompromisse ein. Kurz nach Abschluß dieser Abkommen wurde jedoch die Unzufriedenheit der BdL wieder deutlich ${ }^{266}$.

1954/55: die Verhandlung der EZU-Härtung und neuer Kooperationsformen

Wenn Europa auch mit der Zustimmung zur Konsolidierung einer baldigen Erklärung der Konvertibilität und einer Auflösung der EZU näher gerückt war ${ }^{267}$, so war eine definitive Entscheidung noch nicht gefallen. Zwar wurde im Sommer 1954 eine hochkarätige internationale Ministergruppe - der Ministerausschuß Konvertibilität - eingerichtet, die das zukünftige europäische Handelsund Zahlungssystem verhandeln sollte ${ }^{268}$. Gleichzeitig bestanden aber noch immer prinzipielle Vorbehalte gegen die Konvertibilität. Auch wurden erhebliche Einwände gegen die britischen Vorschläge, dargelegt in einem Memorandum für den Ministerausschu $\beta^{269}$, vorgebracht. Während Frankreich in der ersten Sitzung des Ministerausschusses die Folgen eines Übergangs zur Konvertibilität, namentlich die Spaltung des OEEC-Europas, „in den schwärzesten Farben“ malte, legte Italien ein Memorandum vor, das für den Erhalt der EZU und für ihre schrittweise Härtung eintrat ${ }^{270}$. Auch in der Bundesrepublik gab es weiterhin Zweifel, selbst wenn man den Konsolidierungen zugestimmt hatte. Adenauer wies darauf hin, daß die Wirtschaftspolitik „eine Funktion der Außenpolitik“ sei. Bei den anstehenden internationalen Verhandlungen sollten deshalb keine Ansichten vertreten werden, „die geeignet sind, der europäischen Gemeinschaft zu schädigen“"271. Gleichzeitig ging das Ministerium für Ernährung, Landwirtschaft und Forsten in die Offensive. Es trat dafür ein, „mit den großen weltweiten Planungen vorsichtig zu beginnen und zuerst in Europa die wirtschaftliche und politische Integration durchzusetzen". Ferner zeigten sowohl die BdL als auch das Finanzmini-

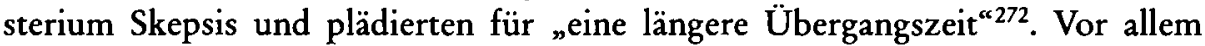
aber distanzierte sich die BdL - mit Unterstützung des konvertibilitätswilligen Wirtschaftsministers - von den britischen Vorschlägen. Der von britischer Seite vorgeschlagene Europäische Fonds traf nicht auf ihre Gegenliebe. Er wurde als

266 Vgl. BBK, B 330/3380, Emminger, Vermerk zu European League for Economic Cooperation, 3.12.1954. Vgl. auch BdL, Monatsbericht Juli 1954, S. $26 \mathrm{ff}$.

${ }^{267}$ Es herrschte die Überzeugung vor, daß dies die letzte Verlängerung der EZU war. Vgl. z.B. von Mangoldt, Stufe; PA AA, Abt. 4/401/23, Hardenberg, Aufzeichnung Ministertagung Konvertibilitätsprobleme, 20.7.1954; BBK, B 330/3171, Emminger, Vermerk, 28.6.1954.

${ }^{268}$ Im Mai 1954 hatte der OEEC-Rat die Konstituierung einer solchen Gruppe beschlossen. Vgl. HAEC, OEEC-TRA 347, Council, Resolution C (54) 131; Cahan, Ministerial Study Group on Convertibility, 10.5.1954. Das ging auf eine Anregung Erhards vom Herbst 1953 zurück. Vgl. Hohmann (Hrsg.), Erhard, S. 391.

269 Vgl. BBK, B 330/3171, Vereinigtes Königreich, Memorandum, GMC (54)1, 4.6.1954.

270 BAK, B 102/55346, Ministerial Examination Group, Minutes of the first session of experts, GMC (54)2, 30.6.1954; BBK, B 330/3171, Emminger, Bericht über die Expertensitzung der OEEC, 23.6.1954. Das italienische Memorandum vom Juli 1954 findet sich in Übersetzung in: BBK, B 330/3380.

271 Kabinettsprotokolle VII (1954), 39. Kabinettssitzung am 13.7.1954, S.309f. Vgl. auch PA AA, Abt. 2/140, Adenauer an Erhard, 5.7.1954.

272 BAK, B 136/7863, Kabinettsausschuß, Protokoll, 13.7.1954. 
„ziemlich sinnlos und überdies gefährlich" angesehen ${ }^{273}$. Wenn die Bundesregierung auch einen solchen Fonds nicht rundheraus ablehnen könne, so betonte die BdL, die europäischen Reserven seien ausreichend. Die Bereitstellung weiterer Kreditmittel sei wohlmöglich sogar kontraproduktiv, denn „die Errichtung eines umfangreichen Fonds mit großzügigen Regeln für die Inanspruchnahme [würde] geradezu als ,Aufforderung zum Tanz' an die verschiedenen Länder wirken müssen, sich bei einem gemeinsamen Konvertibilitätsschritt mindestens für eine gewisse Übergangszeit zurückzuhalten und in dieser Zeit die Mittel des Fonds auszuschöpfen, d.h. sich die Aufrechterhaltung der Liberalisierung möglichst teuer ,abkaufen' zu lassen“274.

Da auch die anderen Punkte des britischen Memorandums - nach einer Übergangszeit sollten die OEEC-Handelsregein in einem reformierten GATT subsumiert werden; eine IMF-GATT-Advisory-Group sollte eingerichtet werden; die EZU-Zwischenfinanzierung sollte durch ein System von Notenbankkrediten ersetzt werden; die Wechselkurspolitik sollte jedem Land überlassen werden - weder in der Bundesrepublik noch in anderen Ländern Zustimmung fanden, konnten auf den ersten beiden Sitzungen des Ministerausschusses keine Beschlüsse gefaßt werden ${ }^{275}$. Da zusätzlich Richard A. Butler, der britische Schatzkanzler, auf der Jahrestagung des IWF im September 1954 betonte, er müsse auch ,innenpolitische Tatbestände" berücksichtigen, und entsprechend die Konvertibilität zurückstellte, erlitten die Verhandlungen über ein zukünftiges Handels- und Währungssystem einen empfindlichen Rückschlag ${ }^{276}$.

Die Bundesregierung und das Zentralbanksystem bedauerten diese Verzögerung $^{277}$. Das Wirtschaftsministerium und das Ministerium für wirtschaftliche $\mathrm{Zu}$ sammenarbeit betonten die „schwerwiegenden Probleme“, die bei Nicht-Erreichen der Konvertibilität auftauchen würden, namentlich die EZU-Überschüs$\mathrm{se}^{278}$. Andererseits aber schickten sich die deutschen Stellen ohne Opposition in diese Verlangsamung. Auch zeigten sie keinerlei Eile, nunmehr ein Handelsund Zahlungssystem für die Zeit nach der Konvertibilitätserklärung zu verabschieden. Erhard - gebunden durch das Gentlemen's Agreement, nur fünf Minuten nach dem Vereinigten Königreich vorzugehen - sagte Butler zu, daß er sich in

${ }^{273}$ BBK, B 330/2030, Könneker an Vocke, 1.7.1954. Zur Haltung des Wirtschaftsministeriums vgl. BAK, B 102/56907, o.V., Vorbereitung einer deutschen Verhandlungslinie, 14.6.1954; BAK, B 136/7863, Kabinettsausschuß, Protokoll, 13.7.1954.

${ }^{274}$ BBK, B 330/78, ZBR, Protokoll, 30.6.1954; BBK, NL Emminger, Vermerke zur Konvertibilität, Emminger, Bemerkungen zu den Vorschlägen für einen Europäischen Fonds, 26.8.1954; BBK, B 330/3171, Emminger, Bericht über Expertensitzung der OEEC, 23.6.1954.

275 Trotzdem wurden die Treffen als Erfolg gewertet, da sich die Positionen angenähert hatten. Vgl. BAK, B 136/7863, l A 1, Vermerk, 5.8.1954; BBK, B 330/78, Emminger, Bericht über die Sitzung der Ministergruppe für Konvertibilitätsfragen, 21.7.1954.

${ }^{276}$ BAK, B 102/55 345, AA, Schnellbrief an BMWi/BMF/BMZ/BdL, 29.9.1954.

277 Der Zentralbankrat sprach davon, daß die Jahrestagung des IWF vom britischen Zögern „überschattet" worden sei. Vgl. BBK, B 330/80, ZBR, Protokoll, 20.10.1954.

${ }^{278}$ Vgl. BAK, B 102/12652, I A 1, Vermerk, 20.9.1954; BAK, B 102/56907, II/5, Aktivierung der OEEC auf dem Gebiet des Zahlungsverkehrs, 15.9.1954. 
Geduld üben würde ${ }^{279}$. Die BdL, unterstützt vom Wirtschaftsministerium, insistierte auf eine retardierende Haltung in den Verhandlungen des Ministerausschusses. Das betraf vor allem die Errichtung eines Europäischen Fonds. Angesichts stetig wachsender europäischer Reserven sah sie die Notwendigkeit eines solchen Kreditsystems nicht ein, opponierte die von einigen Ländern geforderte Ausstattung des Fonds und bestand auf nicht-automatischer Kreditvergabe unter OEEC-Aufsicht. Auch lehnte sie vorerst jegliche definitive Bindung ab. Sie stellte heraus, daß man mit einer vorzeitigen Zustimmung zum Fonds nur dem Vereinigten Königreich die Initiative überlassen würde ${ }^{280}$. Erst im Frühjahr 1955, mit der Aussicht auf eine britische Zustimmung zu einer Bindungs- oder Abbruchklause ${ }^{281}$, ließ die Ablehnung der BdL nach, denn mit einer solchen Abbruchklausel war ihrem Sicherheitsbedürfnis Genüge getan. Emminger stellte nun sogar heraus, daß es wichtig war, ein europäisches Forum zu erhalten: „Nach dem Aufhören der heutigen EZU ist ein neues Forum für die währungspolitische Zusammenarbeit und für ad-hoc-Hilfen auf multilateraler Basis sehr nützlich, wenn nicht sogar unentbehrlich." ${ }^{282}$

Auf dieser Grundlage konnten die Beratungen über den Europäischen Fonds abgeschlossen werden. Am 16. April 1955 legte das EZU-Direktorium dem OEEC-Rat den entsprechenden Bericht vor. Dieser empfahl die Errichtung eines Europäischen Fonds, der nicht-automatische Kredite bis zu einer Gesamthöhe von 600 Mio. RE vergeben konnte ${ }^{283}$. Wenn damit auch eine Empfehlung ausgesprochen worden war, war noch unsicher, ob sie realisiert werden würde. Die Verwirklichung des Europäischen Fonds hing davon ab, ob man sich rechtzeitig über die weiteren Diskussionspunkte - die Handelsregeln, den Ersatz der EZUZwischenfinanzierung und des EZU-Kompensationsmechanismus sowie die EZU-Verlängerung - würde einigen können.

Die BdL stand einer erneuten Verlängerung der EZU zu Anfang zwiespältig gegenüber. Schließlich hatte sie sich schon mit der Idee einer baldigen EZU-Liquidation angefreundet. Ganz in diesem Sinne stellte Emminger fest, daß die

279 Vgl. PRO, T 236/3519, Treasury, Note of a meeting of the Chancellor and Erhard, 30.9.1954. Aus dem Drängen Butlers auf die Einhaltung dieses Gentlemen's Agreement im Juli 1955 und aus den Reaktionen Adenauers und Blüchers ergibt sich, daß es problematisch war, diese Absprache zu vernachlässigen. Vgl. BAK, B 136/7863, Blücher an Adenauer, 20.6.1955; Haenlein an BMF/ BMWi, 28.7.1955.

280 Vgl. BAK, B 102/55340, BdL an Blücher/Erhard/Schäffer, 5.1.1955; V A II, Vorbereitung der Sitzung des Ministerausschusses, 4.1.1955, Gocht an Erhard, 8.1.1955. Vgl. auch BBK, B 330/82, ZBR, Protokoll, 5.1.1955.

281 Die Abbruchsklausel besagt, daß das EWA bzw. der Europäische Fonds nur in Kraft treten kann, wenn Unterzeichner, die 50\% der Beiträge stellen, der Beendigung der EZU und der Anwendung des EWA zustimmen. Vgl. den Text des EWA-Abkommens, Art. 27, in: BGBL 1959 II, S. $293 \mathrm{ff}$.

282 BBK, B 330/1273, Emminger, Vermerk, 10.3.1955. Vgl. auch BBK, B 330/3314, BdL, Vermerk betreffend Aufbau des Europäischen Fonds, 21.4.1955; BBK, B 330/1310, Emminger, Vermerk zu Bericht des EZU-Direktoriums, 30.4.1955.

${ }^{283}$ Vgl. HAEC, OEEC-TRA 344, EPU Board Report, The Renewal of the EPU and the Establishment of a European Fund, C (55)91, 22.4.1955. 
EZU „den Stab bei der Herstellung der wirtschaftlichen Einheit Europas an andere abgeben [muß] ${ }^{\alpha 284}$. Andererseits aber schätzte die Bank die EZU noch immer, so daß sie bald der Verlängerung der EZU wieder zusätzliche positive Seiten abgewann. Mit Blick auf den deutschen NATO/WEU-Beitritt und die damit zusammenhängenden Rüstungsausgaben meinte Emminger: „Es liegt im wohlverstandenen deutschen Interesse, wenn die EZU noch bis zum Auftreten dieser rüstungsbedingten deutschen Defizite aufrechterhalten wird, damit wir unsere angesammelten liquiden EZU-Guthaben [...] noch hierfür einsetzen können. Eine vorzeitige Beendigung der EZU würde bedeuten, daß wir diese EZU-Forderungen gemäß dem EZU-Abkommen auf mehrere Jahre konsolidieren, andererseits aber die danach gegenüber EZU-Ländern anfallenden Defizite in konvertibler Währung zu begleichen hätten. “" 85 Auch Vocke plädierte im Dezember 1954 für eine Verlängerung der EZU „tel quel“, ohne sich den belgischen, holländischen und schweizerischen Forderungen nach Härtung auf 75 Prozent anzuschließen ${ }^{286}$. Erst im Verlauf der Verhandlungen stimmte der Zentralbankrat, wenn auch nicht hundertprozentig überzeugt, einer Härtung auf 75 Prozent zu, „weil sie der von deutscher Seite vertretenen Linie der Härtung der EZU entspricht“. Dabei warnte er zwar vor dem Risiko, „daß die Bundesrepublik für den Fall einer Umkehr ihrer Zahlungsposition mehr Gold einzuschießen habe, als sie bei Aufbau der Überschußposition erhalten habe". Das mußte jedoch nach Auffassung des Zentralbankrates hingenommen werden ${ }^{287}$.

Die möglichen, rüstungsbedingten Defizite waren keineswegs das einzige Motiv für das EZU-Plädoyer der Bank. Genauso wichtig war ihr, daß mit der EZU ein Kompensationsmechanismus erhalten bleiben würde, der ihres Erachtens mehrere Vorteile hatte. Zum ersten würde dadurch das "leidige Problem des Auseinanderfallens in konvertible und nicht-konvertible Länder" gar nicht entstehen $^{288}$. Zum zweiten bliebe damit die EZU-Interimsfinanzierung der Notenbanken erhalten. Ein britischer Vorschlag, diese Zwischenfinanzierung durch kurzfristige Swaps zu ersetzen - laut BdL ein "gefährlicher" Vorschlag, da er die Kreditvergabe erhöhen würde -, würde damit gegenstandslos ${ }^{289}$. Zum dritten

${ }^{284}$ BBK, NL Emminger, Vorträge, Emminger, Manuskript zur EZU, November 1954. Vocke lehnte die Veröffentlichung dieses Vortrages ab.

${ }^{285}$ BBK, B 330/3380, Emminger, Vermerk, 29.10.1954. Vgl. auch BBK, B 330/1314, 6 a/601, Entwurf eine Stellungnahme, 2.11.1954. Vgl. auch BBK, NL Emminger, Vorträge, Emminger, Auf dem Weg zur Konvertibilität, August 1954. Auch die Veröffentlichung dieses Vortrages lehnte Vocke ab.

${ }^{286}$ BBK, B 330/2056, Direktorium, Protokoll, 2.12. 1954. Diese deutsche Haltung hatte die Bank von England richtig vorausgesehen. Vgl. BoE, OV 34/40, Heasman, German attitude towards EPU, 12.11.1954; BoE, OV 46/18, Heasman an Parsons, 11.12.1954.

${ }^{287}$ BBK, B 330/83, ZBR, Protokoll, 2.3.1955.

${ }^{288}$ BBK, B 330/83, ZBR, Stenogramm, 2.3.1955.

${ }^{289}$ BBK, NL Emminger, Vermerke zur Konvertibilität, Emminger, Stellungnahme zu dem Boltonund Ansiaux-Plänen, 5.11.1954; BBK, NL Emminger, Notenbankvereinbarungen, Emminger an Vocke, 6.10.1954. Vgl. auch BoE, OV 46/65, Bolton an Cobbold, 2.10.1954; BoE, G 1/507, Bolton an Cobbold, 25.9.1954. 
würde aufgrund der EZU-Wechselkursgarantie das Wechselkursrisiko vermindert und eine gewisse Stabilität der Wechselkurse garantiert ${ }^{290}$. Zum vierten würden mit der EZU die bisherigen OEEC-Handelsregeln in Kraft bleiben.

Aus diesen Gründen trat die BdL - zusammen mit anderen europäischen Ländern und, abgesehen vom Wirtschaftsministerium, einer Meinung mit der Bundesregierung - für die Idee einer "Metamorphose" der EZU ein. Die EZU solle nicht liquidiert, sondern schrittweise in den Europäischen Fonds überführt werden. Die Möglichkeit der Gewährung von EZU-ad-hoc-Krediten sollte ausgebaut werden, während die automatischen Kreditlinien gleichzeitig schrittweise abzubauen waren ${ }^{291}$. Problematisch war allerdings, daß sich das Vereinigte Königreich, das inzwischen einer Härtung der EZU auf 75 Prozent zustimmte, einer solchen Metamorphose entgegenstellte. Deshalb mußten im Frühjahr 1955 ein neuer Kompensationsmechanismus und zukünftige Handelsregeln diskutiert werden.

Bei diesen Verhandlungen, die zum Teil im Ministerausschuß, zum Teil unter Notenbankvertretern geführt wurden, bildete sich schnell „eine überraschend starke kontinentaleuropäische Front" heraus. Das Vereinigte Königreich lehnte einen multilateralen Abrechnungsmechanismus ab, da dies durch Devisenarbitrage besorgt werden könne. Darüber hinaus wollte es sich in der Wechselkurspolitik nicht binden. Demgegenüber befürworteten die kontinentaleuropäischen Länder unter der Führung der Schweiz ein Kompensationssystem. Dabei visierten sie auch eine Verankerung der Wechselkursstabilität $a^{292}$. Die deutsche Seite sympathisierte weitgehend mit diesen Forderungen: Wenn auch das Wirtschaftsministerium solche "perfektionistische" Bemühungen opponierte ${ }^{293}$, so hießen die BdL und die Bundesvertretung in Paris diese Pläne für gut: „Ein Kompromiß, der mindestens Frankreich - und damit wohl auch Italien - zum Mitgehen bei der ,non-resident convertibility' bringt, ist unter allen Umständen anzustreben, da andernfalls die Gefahr eines teilweisen Rückfalls in bilaterale Zahlungspraktiken sehr groß wäre. [...] Unter diesem Gesichtspunkt sei insbesondere auch unsere Sympathie für eine zeitlich begrenzte Fortdauer der multilateralen

${ }^{290}$ Das Wechselkursrisiko und die Angst vor flexiblen Wechselkursen standen im Zentrum der Aufmerksamkeit der BdL. Vgl. BBK, NL Emminger, Notenbankvereinbarungen, Emminger, Bericht über eine Diskussion von Notenbank-Experten über Fragen des europäischen Zahlungsverkehrs, 17.11.1954; Emminger an Vocke, 6.10.1954.

291 Für die Haltung des Zentralbankrates vgl. BBK, B 330/83, ZBR, Protokoll und Stenogramm, 2.3.1955. Für das Ministerium für wirtschaftliche Zusammenarbeit und die Bundesvertretung in Paris vgl. BAK, B 126/7743, BMZ an BMW//AA/BdL, 24.5.1955. Für die Haltung der anderen Länder vgl. BBK, B 330/82, Emminger, Bericht über die Sitzung des Stellvertreter-Ausschusses am 7./8.12.1954, 9.12.1954. Für das Wirtschaftsministerium vgl. BAK, B 102/12653, I A 1, Vermerk, 22.4.1955. Auf die Frage, wie die deutsche Delegation ihre Ideen mit Erhards Äußerungen vereinbarte, antwortete von Mangoldt, "that one of their objectives at the moment was to keep Erhard's mouth closed“. BoE, OV 46/66, Parsons, Note on Uniscan meeting, 30.3.1955.

${ }^{292}$ BBK, B 330/3172, Emminger, Bericht über die Sitzung der Stellvertreter, 15.5.1955.

293 BAK, B 102/12653, I A 1, Vermerk, 22.4.1955. 
Kompensation in Europa bei Aufhören der gegenwärtigen EZU zu sehen. Selbst wenn sachlich nicht allzu viel drin stecken würde, wäre es von Vorteil. “294

Erst Ende Juni 1955 näherten sich die Fronten an. Nur wegen der Aussicht, die britischerseits schon akzeptierte Verlängerung einer auf 75 Prozent gehärteten EZU durch eine insistierende Haltung zu gefährden, war die BdL zu einem Kompromiß bereit. Der Zentralbankrat stimmte in seiner Sitzung am 22./ 23. Juni einem System des Zahlungsausgleichs zu, auch wenn sich damit das Vereinigte Königreich nicht auf feste Wechselkurse verpflichten ließ ${ }^{295}$. Der Meinungswandel der Bank ist vor allem dadurch zu erklären, daß die britische wirtschaftliche Situation ein Inkrafttreten des EWA vorerst unwahrscheinlich mach$\mathrm{te}^{296}$, während ein Entgegenkommen die inzwischen angestrebte Verlängerung und Härtung der EZU, die zeitweise durchaus gefährdet erschien, ermöglich$t^{297}$. Entsprechend stellte Emminger in einer Sitzung des Zentralbankrates fest, daß das EWA lediglich ein „Schubladenbeschluß“ sei, während die auf 75 Prozent gehärtete EZU vermutlich noch einige Jahre unangefochten weiterbestehen würde ${ }^{298}$.

Aufgrund dieser Einschätzung stimmte die BdL auch den neuen Handelsregeln - zeitlich unbeschränkte Geltung der OEEC-Handelsregeln mit einigen Modifikationen und Bedingungen - zu, selbst wenn ihres Erachtens die Durchsetzung dieser Regeln „wahrscheinlich nur ein Pyrrhussieg“ war ${ }^{299}$.

\section{Lockerung und Umgestaltung der deutschen Devisenbewirtschaftung}

In den internationalen Verhandlungen hatte die BdL eine konvertibilitätsskeptische und retardierende Haltung eingenommen. Das war aber nur eine Seite der Medaille. Auf der anderen Seite - bei der allmählichen Lockerung der Devisenbewirtschaftung und bei der schrittweisen Herstellung der Inländer-Konvertibilität - nahm sie einen anderen Standpunkt ein. Von Anfang an stellte sie heraus, daß die Bundesrepublik auch gewisse unilaterale Maßnahmen ergreifen könne. Nachdem sie ihre anfängliche Angst vor „einem Sprung in das Chaos“ überwunden

294 BBK, B 330/2020, Emminger an Vocke, 24.5.1955.

295 Vgl. BBK, B 330/86, ZBR, Stenogramm, 22./23.6. 1955. Für die frühere Position vgl. BBK, B 330/ 84, ZBR, Protokoll, 16.3.1955.

296 Anfang 1955 zeigten sich erneut britische Zahlungsbilanzschwierigkeiten. Vgl. Shonfield, Policy, S. $199 \mathrm{ff}$.

${ }^{297}$ Der Zentralbankrat befürchtete, daß die anderen Länder wegen der ausgesprochen hohen deutschen Überschüsse vom Sommer 1955 in letzter Minute von der Härtung und Verlängerung der EZU Abstand nehmen könnten. Vgl. BBK, B 330/86, ZBR, Stenogramm, 22./23.6. 1955.

298 BBK, B 330/2020, Emminger an Vocke, 21.6. 1955; BBK, B 330/87, ZBR, Stenogramm, 3.8.1955.

299 BBK, B 330/86, ZBR, Stenogramm, 22./23.6. 1955. Die modifizierten Handelsregeln erlaubten die Suspendierung der Liberalisierung nur, wenn sich die Gesamtzahlungsbilanz eines Landes negativ entwickelte. Für die Einführung dieser Handelsregeln war die Existenz eines multilateralen Abrechnungssystems Bedingung. Emminger befürchtete, daß Frankreich diesen Passus restriktiv auslegen würde. Zu den handelspolitischen Diskussionen und Entwicklungen vgl. Asbeek Brusse, Tariff Plans, S. $202 \mathrm{ff}$. 
hatte ${ }^{300}$, sah sie hier erhebliche Möglichkeiten. Im folgenden strebte sie die Umgestaltung und Lockerung der deutschen Devisenbewirtschaftung "so rasch [...] als devisenpolitisch vertretbar " $\mathrm{an}^{301}$. Dieser Politik lag ein dreifaches Motiv zugrunde: Zum ersten wollte die BdL durch die Aufhebung der devisenpolitischen Restriktionen schrittweise die Inländer-Konvertibilität herstellen, da das Vorteile für die deutsche Wirtschaft hätte. Zum zweiten sah sie eine solche Politik als eine Good Creditor Policy an, die anderen Ländern Fortschritte auf ihrem Weg zur Konvertibilität erlauben würde ${ }^{302}$. Zum dritten sah sie sich veranlaßt, britischen Maßnahmen zu folgen, um gegebenenfalls auf eine britische Konvertibilitätsinitiative vorbereitet $\mathrm{zu} \operatorname{sein}^{303}$.

\section{OEEC-Liberalisierung}

Schon zu Anfang der deutschen Konvertibilitätsdiskussionen hatte sich die BdL als entschiedene Befürworterin der OEEC-Liberalisierung profiliert, wobei sie eine strikte Gegenposition zu Erhard eingenommen hatte. Auch im weiteren Verlauf sprach sie sich für eine stetige Erhöhung der OEEC-Liberalisierung aus. Als im April 1953 eine Liberalisierung des Warenverkehrs von über 90 Prozent erreicht war, dehnte sie ihr Plädoyer sogar - wenn auch nach anfänglichem Zögern - auf die Liberalisierung der unsichtbaren Transaktionen aus. Die Motive hierfür waren vor allem, daß die Bank mit diesen Maßnahmen der deutschen Gläubigerposition in der EZU entsprechen wollte. Dadurch würde die EZU funktionsfähig erhalten ${ }^{304}$.

\section{Devisen-Monopol und -Arbitrage}

Aber nicht nur in bezug auf den Waren- und Dienstleistungsverkehr, sondern auch in bezug auf ihr Devisenmonopol verfügte die BdL. Lockerungen. Noch bevor die Parität der DM offiziell beim IWF festgesetzt worden war ${ }^{305}$, ergriff die BdL die Initiative, um „unverzüglich“ nach der Kursfestsetzung die Grundsteine eines Devisenmarktes legen zu können, der „eine beweglichere, marktkonformere Notierung“ erlauben sollte ${ }^{306}$. Dabei war die Möglichkeit der Devisenarbitrage zwischen der DM, dem holländischen Gulden, dem schweizerischen und belgischen Franken, möglichst auch dem Pfund vorgesehen. Da auch die Bank

\footnotetext{
300 BBK, B 330/57, ZBR, Protokoll, 11.6.1952.

${ }^{301}$ BBK, B 330/13073(2), Emminger, Stand der Konvertibilitätsgespräche, 30.6.1953.

${ }^{302} \mathrm{Vgl}$. ebenda.

${ }^{303}$ Im Rahmen des Collective Approach verfolgte das Vereinigte Königreich die Annäherung an die defacto-Konvertibilität des Pfundes. Es baute Handels- und Zahlungsrestriktionen schrittweise ab, eröffnete die Londoner Rohstoffmärkte und verfügte am 22.3.1954 eine Vereinheitlichung der unterschiedlichen Sterlingkonten. Vgl. Rees, Britain, S. $155 \mathrm{ff}$.; Fforde, Bank of England, S. $492 \mathrm{ff}$.

${ }^{304} \mathrm{Vgl}$. S. 134f. und $149 \mathrm{f}$.

${ }^{305}$ Die Bundesrepublik war im Sommer 1952 Mitglied des IWF geworden. Die Parität der DM wurde aber erst Anfang 1953 festgesetzt. Vgl. Bulletin des Presse- und Informationsamtes, Nr.27, 10.2.1953, S.220.

${ }^{306}$ BBK, B 330/5461, Treue, Vermerk, 8.1.1953; BBK, B 330/64, ZBR, Protokoll, 7.1.1953. Vgl. auch BBK, NL Emminger, Vermerke zur Konvertibilität, Emminger, Vermerk, 3.12.1952.
} 
von England eine multilaterale Devisenarbitrage zwischen einigen EZU-Währungen anvisierte ${ }^{307}$, darüber hinaus inzwischen einer Notierung der DM in London zustimmte ${ }^{308}$, wurde im Frühjahr 1953 der multilaterale Devisenhandel zwischen Pfund, DM, holländischem Gulden, schwedischer Krone, französischem, belgischem und schweizerischem Franken aufgenommen ${ }^{309}$. Wenngleich sich der Zentralbankrat dagegen verwahrte, dies als einen „eigentlichen Schritt zur Konvertibilität" anzusehen, war damit eine Bresche in das Devisenmonopol der BdL geschlagen $^{310}$ : Exporteure konnten nun Währungskonten einrichten, die Ablieferungspflicht für Devisen wurde gelockert; die Belassungsfrist wurde anfänglich auf zehn Tage, später auf sechs Monate festgesetzt. Die Wirtschaft wie die Geschäftsbanken wurden so wieder in den Devisenhandel eingebunden. Das hatte das angenehme und sehr geschätzte Nebenergebnis, daß die EZU-Überschüsse zu Zeiten weniger zutage traten. Im August 1953 versuchte die Bank, innerhalb der EZU-Quote von 650 Mio. RE zu bleiben, indem sie die Möglichkeiten der Devisenbelassung bei den Außenhandelsbanken vergrößerte ${ }^{311}$.

\section{Dollar-Liberalisierung}

Bei den Diskussionen über die Konvertibilität und die Lockerung der Devisenbewirtschaftung war auch die Handelsliberalisierung zum Dollarraum ein wichtiger Punkt. Schon im Frühjahr 1953 befürwortete Hans Karl von Mangoldt, deutscher Vertreter im EZU-Direktorium, eine Liberalisierung zum Dollarraum. Die Bundesrepublik solle bei ihrer zufriedenstellenden Reservensituation die Möglichkeit des billigen Rohstoffbezugs ausnutzen ${ }^{312}$. Kurze Zeit später plädierten auch Erhard und deutsche Wirtschaftsvertreter für eine Dollarliberalisierung. $\mathrm{Da}$ die Regelung der Einfuhranrechte ausgelaufen war, suchten sie andere Möglichkeiten, die Einfuhr aus dem Dollarraum zu erleichtern ${ }^{313}$.

Die BdL stand der Dollarliberalisierung anfangs skeptisch und abwartend gegenüber. Sie bestand darauf, auch die Gründe, die gegen die Liberalisierung der Dollarimporte sprachen, in die Überlegungen einzubeziehen ${ }^{314}$. Zum ersten wür-

\footnotetext{
${ }^{307}$ Vgl. BoE, OV 46/13, Bridge, Multilateral Arbitrage, 21.11.1952.

${ }^{308} \mathrm{Vgl}$. BoE, OV 34/65, Bridge, The DM, 17.12.1952. Trotz heftigen Insistierens deutscherseits hatte das Vereinigte Königreich im Jahre 1949/50 eine Notierung der DM abgelehnt. Vgl. hierzu BoE, OV 34/56, Rootham, Note, 22.8.1950 und die Unterlagen über die Verhandlung eines deutschbritischen Zahlungsabkommens in BoE, OV 34/50 und OV 34/58.

${ }^{309} \mathrm{Vgl}$. BAK, B 102/55 333, BdL an BMWi, 8.5.1953; Kühne, Regelungen, S. $300 \mathrm{ff}$.

310 BBK, B 330/64, ZBR, Stenogramm, 21./22.1.1953; BBK, B 330/79, ZBR, Protokoll, 8. 9.1954. Vgl. auch BBK, B 330/5461, Auslandssekretariat, Vermerk, 4.3.1953. Vgl. auch Kühne, Regelungen, S. 300, der diese Maßnahmen als "von erheblicher Bedeutung für die Normalisierung des deutschen Auslandszahlungsverkehrs" ansieht.

311 Vgl. BBK, B 330/2056, Direktorium, Protokoll, 16.7.1953 und 13.8.1953.

312 Vgl. BAK, B 102/11 579, von Mangoldt, Abbau der diskriminierenden Einfuhrbeschränkungen gegenüber dem Dollarraum, 25.4.1953.

313 Vgl. BBK, B 330/2056, Direktorium, Protokoll, 18.6.1953. Zu den Einfuhranrechten vgl. Fußnote 82.

${ }^{314}$ Vgl. BBK, B 330/70, ZBR, Stenogramm, 24.6.1953.
} 
de sich - so befürchtete die Bank - eine Dollarliberalisierung nachteilig auf die Beziehungen zu anderen Zahlungsräumen auswirken, weil sich ein Abbau der bilateralen Guthaben dadurch schwieriger stellen würde. Auch könnte sich durch eine Liberalisierung der Dollareinfuhr die deutsche Überschußposition innerhalb der EZU akzentuieren ${ }^{315}$. Zum zweiten - so betonte sie - hinge die Dollarliberalisierung nicht nur von der eigenen Position ab, sondern vor allem auch davon, inwieweit die deutschen Überschüsse gegenüber anderen Ländern konvertibel seien ${ }^{316}$. Zum dritten müsse man sich durch ein gemeinsames Vorgehen der OEEC-Länder "gewisse amerikanische Zugeständnisse" einhandeln ${ }^{317}$. Da einige EZU-Mitglieder erhebliche Vorbehalte gegen eine Liberalisierung der Dollareinfuhr hatten, hieß dieses Plädoyer der Bank, daß vorerst keine Schritte unternommen werden sollten.

Erst als im November 1953 die USA, die bisher die OEEC-Liberalisierung anstandslos akzeptiert hatten, auf einen Abbau der Beschränkungen zum Dollarraum drängten ${ }^{318}$, befürwortete auch die BdL ein schrittweises Vorgehen. Dabei legte sie besonderen Wert auf die Liberalisierung der Rohstoffe und Grundnahrungsmitte ${ }^{319}$. Auf dieser Grundlage wurde im Februar 1954 die erste Dollarfreiliste in Kraft gesetzt, wodurch 51,9 Prozent der Einfuhrwerte von 1953 liberalisiert wurden. Im Verlauf des Jahres 1954/55 erhöhte die Bundesrepublik diesen Satz auf 68,1 Prozent. Zwar fanden diese Erhöhungen nicht immer sofort die Zustimmung der Bank. Letztendlich aber stimmte sie nach einigen Diskussionen zu, denn die inzwischen auf $10 \mathrm{Mrd}$. DM angewachsenen konvertiblen Währungsreserven sprachen eindeutig für eine weitere Liberalisierung. Hermann Tepe, Präsident der Landeszentralbank Bremen, führte in der Sitzung des Zentralbankrates am 2.Februar 1955 aus: „Ich wollte nur feststellen, ob der in der letzten Sitzung prinzipiell gefaßte Beschluß, sich der Liberalisierung entgegenzustellen, etwas modifiziert werden soll. Es ist mir etwas wesentlich, daß wir nicht principiis obsta sagen: Wir sitzen auf unseren Dollars wie der Drache auf dem Nibelungenschatz. ${ }^{\text { } 320}$

Als Ergebnis dieser Politik wuchs der prozentuale Anteil der Dollarimporte von 13,5 Prozent (1953) auf 17,4 Prozent (1956) an. In der gleichen Zeit ging die Einfuhr aus dem EZU-Raum von 66,3 Prozent (1953) auf 60,1 Prozent (1956) zurück ${ }^{321}$.

${ }^{315}$ Vgl. BBK, B 330/70, ZBR, Protokoll, 24.6.1953; BBK, NL Emminger, Konvertibilität/Abbau der Devisenzwangswirtschaft II 1953, Emminger, Zum Thema Konvertibilität, 8.4.1954.

316 Vgl. BBK, NL Emminger, Konvertibilität/Abbau der Devisenzwangswirtschaft II 1953, Emminger, Abbau der Devisenrestriktionen, 12.2.1954.

317 BBK, B 330/2056, Direktorium, Protokoll, 18.6.1953.

318 Während Erhards Amerika-Reise sprachen die Amerikaner die Dollarliberalisierung immer wieder an. Vgl. die Protokolle der Besprechungen in: NARA, RG 59 862A.00/11-2353 bis 862A.00/112553.

319 Vgl. BBK, NL Emminger, Konvertibilität/Abbau der Devisenzwangswirtschaft II 1953, o.V., Konvertierbarkeit der Währungen von Deutschland aus gesehen, o. D. [Januar 1954]

320 BBK, B 330/83, ZBR, Stenogramm, 2.2.1955. Vgl. Deutsches Geld- und Bankwesen, S. 348.

321 Vgl. Tabelle 5. 


\section{Die Beko-Mark und der Abbau des Bilateralismus}

Im Jahre 1952 waren mit der Entwicklung deutscher Überschüsse zu den bilateralen Verrechnungsländern und vor allem mit dem Brasilienfall die Probleme des Bilateralismus deutlich geworden. Nachdem das deutsche Mitglied im EZUDirektorium, Hans Karl von Mangoldt, im Sommer 1953 den Abbau des Bilateralismus angesprochen, gleichzeitig der IWF während der jährlichen Konsultationen entsprechende Maßnahmen gefordert hatte ${ }^{322}$, griff im Herbst 1953 auch die BdL das Problem des Abbaus des Bilateralismus auf. Sie wollte „den Boden für eine Konvertibilität [...] vorbereiten" und forderte den Wirtschaftsminister auf, bei einzelnen Ländern, mit denen bilateral abgerechnet wurde, den Übergang zum freien Dollarverkehr einzuführen ${ }^{323}$. Das jedoch war nicht einfach. Zum einen opponierte die deutsche Exportindustrie derartige Maßnahmen ${ }^{324}$, zum anderen zeigten bilaterale Verhandlungen, daß die Umstellung der Zahlungsabkommen auf Dollarabrechnung bei den Abkommenspartnern auf Vorbehalte stieß. Nur Mexiko und Kolumbien willigten ein, zur Dollarabrechnung überzugehen ${ }^{325}$.

Da bilaterale Verhandlungen auf Schwierigkeiten trafen, verlegte sich das BdLDirektorium auf unilaterale Maßnahmen. Im November 1953 schlug es vor, die deutsche Devisenbewirtschaftung kurzerhand durch eine Mitteilung an die AuBenhandelsbanken zu „vereinfachen“. Der Zentralbankrat stimmte diesem Vorschlag zu. Die BdL informierte daraufhin das Wirtschaftsministerium, daß sie die Vielzahl der DM-Sonderkonten für Ausländer ${ }^{326}$ in zwei Kontenarten - freie DM-Konten und beschränkt konvertierbare DM-Konten - umwandeln wollte. Dabei sollten Zahlungen aller Art mit den Guthaben dieser Konten erlaubt sein, wobei allerdings Guthaben der beschränkt konvertierbaren Konten nicht auf frei konvertierbare Konten übertragen werden konnten. Wenngleich die Bank nur Kann-Vorschriften vorschlug und außerdem vorerst vorsah, die laut den jeweiligen Zahlungsabkommen vorgesehenen DM-Konten ausländischer Zentralbanken von dieser Regelung auszunehmen, zielte diese einfache, banktechnische Maßnahme auf eine grundlegende Umgestaltung der deutschen Devisenbewirt-

322 Vgl. den Kieler Vortrag von von Mangoldt, in: BdL, Auszüge aus Presseartikeln, Nr. 80/53; BAK, B 102/11 579, Fischer, Konsultationen mit dem IWF 1953, 19.4.1954.

${ }^{323}$ Vgl. BBK, B 330/73, BdL an BMW, 30.10 .1953 ; BBK, B 330/2056, Direktorium, Protokoll, 8.10.1953.

${ }^{324}$ Vgl. BBK, B 330/3341, BDI, Memorandum DM-Ausländerkonten, 19.1.1955, Boden (BDI) an Vocke, 20.1.1955.

${ }^{325}$ Die Zahlungsabkommen mit Mexiko und Kolumbien wurden zum 1.6.1952 bzw. zum 1.10.1954 auf Dollar umgestellt. Chile wollte an seinem bilateralen Abkommen festhalten. Vgl. BdL, Geschäftsbericht 1954, S. 103; BBK, B 330/73, ZBR, Protokoll, 11.11 .1953 und BBK, B 330/74, ZBR, Stenogramm, 16.12.1953.

${ }^{326}$ Es gab eine Vielzahl von DM-Sonderkonten wie Agenten-Konten, Luftfahrtkonten, Exportabwicklungskonten, Dienstleistungsentgeltkonten, Vertreterkonten, Sperrmarkkonten für ausländische Handelsfirmen, Dienstbezügekonten. Ferner gab es noch Sperrmarkkonten und DM-Konten ausländischer Zentralbanken oder Verrechnungsinstitute. Alle diese Sonderkonten waren genehmigungspflichtig und unterlagen erheblichen Beschränkungen. 
schaftung. Da vorgesehen war, daß einerseits deutsche Importeure - sofern es ihren Handelspartnern angenehm war und es ihnen von ihren nationalen Devisenbehörden erlaubt wurde - ihre Schulden durch Zahlung auf diese Konten begleichen konnten, andererseits ausländische Importeure mit diesen Guthaben ihre Bezüge aus der Bundesrepublik bezahlen konnten, würden damit die Keime von zwei Zahlungskreisen - dem Kreis der konvertierbaren DM und dem Kreis der beschränkt konvertierbaren DM oder Beko-Mark - gelegt werden ${ }^{327}$. Dadurch käme der DM im internationalen Zahlungsverkehr aufgrund ihrer erhöhten Transferierbarkeit eine größere Bedeutung zu. Vor allem bot sich damit die Gelegenheit, den strikten Bilateralismus zu lockern.

Die Initiative der BdL stieß auf erhebliche Bedenken. Der Finanzminister warf ein, daß es sich „nicht um bloße Änderungen zahlungstechnischer Verfahrensregeln" handele. Er verlangte nicht nur, daß sein Ressort zu den Beratungen hinzugezogen würde - er lehnte auch eine solche Maßnahme aus prinzipiellen Gründen $a b$. Unter dieser neuen Regelung würde der deutsche Export leiden. Das bilaterale Verrechnungssystem könne Guthaben verlieren. Möglicherweise würde der Bund verstärkt in Anspruch genommen werden ${ }^{328}$. Seine Einwände wurden aber nicht akzeptiert. Die Bank hob die Vorteile dieser Regelung wie höhere Verwendbarkeit der DM und mögliche Preiszugeständnisse seitens der Zulieferer hervor. Den Ausfuhrmöglichkeiten insgesamt werde durch die neuen Bestimmungen kein Abbruch getan. Die Bundesrepublik befände sich gegenüber allen bilateralen Ländern in einer Gläubigerposition, so daß die bisher im Rahmen bilateraler Abkommen verwendeten Guthaben auf jeden Fall zu einem Abbau der Gläubigerposition beitragen würden ${ }^{329}$. Unterstützt wurde die Bank in ihrem Plädoyer für diese neue Regelung vom Wirtschaftsministerium. Wie das Finanzministerium kritisierte es zwar die Vorgehensweise der BdL und bestand auf einem Runderlaß Außenwirtschaft, an dem es mitwirken würde. Darüber hinaus stimmte es aber der vorgeschlagenen Regelung zu. Angesichts der von der BdL vorgeschlagenen sachlichen Begrenzung lägen Bedenken nicht vor. Dieser Schritt müsse getan werden, „um auf dem Wege zur freien Währungsaustauschbarkeit wenigstens ein Stück weiter zu kommen “ ${ }^{\text {“30. }}$ So konnte am 22. März 1954 der Runderlaß Außenwirtschaft Nr.24/54 verabschiedet und die von der BdL vorgeschlagene neue Kontenregelung eingeführt werden $^{331}$.

${ }^{327}$ Vgl. BBK, B 330/2056, Direktorium, Protokoll, 5.11.1953; BBK, B 330/74, ZBR, Protokoll und Stenogramm, 16.12.1953, Vocke an Bernard, ZBR-Vorlage, 24.11.1953; BAK, B 102/57551, BdL an $\mathrm{BMWi}, 30.11 .1953$.

$328 \mathrm{BBK}, \mathrm{B} 330 / 3241, \mathrm{BMF}$ an BdL/BMWi/BMZ, 10.1.1954.

329 V.gl. BBK, B 330/2043, BdL an BMF, 4.3.1954.

${ }^{330}$ BAK, B 102/57551, Felsch, Vermerk, 10.12.1953; V A 9a an V A 8, 18.2.1954.

${ }^{331} \mathrm{Zu}$ den technischen Details vgl. Kühne, Regelungen, S. $387 \mathrm{ff}$. 
Tabelle 9: DM-Verpflichtungen der Geschäftsbanken 1954-1956

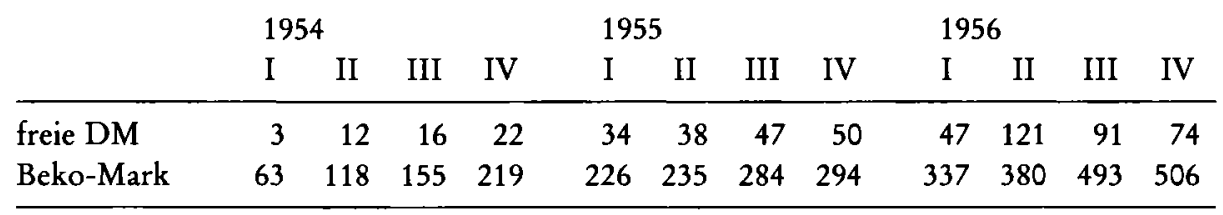

Anmerkung: Alle Angaben in Mio.DM.

Quelle: BdL, Monatsberichte, verschiedene Ausgaben.

Die neu eingeführten Konten erfreuten sich von Anbeginn regen Zuspruchs. Wie Tabelle 9 zeigt, waren schon Ende März 1954 auf den Beko-Mark-Konten Guthaben in Höhe von über 50 Mio. DM aufgelaufen.

Auf der Grundlage dieses regen Interesses konnte im weiteren Verlauf diese Abrechnungsmöglichkeit weiter ausgebaut und viele bilaterale Zahlungsabkommen nach und nach auf Beko-Mark umgestellt werden. Nach zum Teil heftigen Auseinandersetzungen mit dem Wirtschaftsministerium und dem Bundesverband der Deutschen Industrie, die in spezifischen Fällen bemängelten, dadurch würde der Einfluß der deutschen Handelspolitik auf den Warenverkehr mit den bilateralen Ländern zurückgeschraubt ${ }^{332}$, wurde zum 1.Oktober 1954 die Beko-Mark im Zahlungsverkehr mit Finnland, zum 16. Mai 1955 im Zahlungsverkehr mit Spanien eingeführt. Es folgten Brasilien, Paraguay, Japan, Ungarn und Uruguay, während mit Ecuador die Dollarabrechnung vereinbart wurde. Insgesamt wurde bis Ende 1956 die Zahl der bilateralen Zahlungsabkommen von anfänglich 18 auf 3 zurückgeführt. Die von der BdL gewährten Swing-Kredite sanken von 208 Mio. Dollar auf knapp 1 Mio. Dollar ${ }^{333}$. Die Bank von England beobachtete dieses schrittweise deutsche Vorgehen argwöhnisch. Sie bezeichnete es als „multilateralism where it suits them, bilateralism where it does not ${ }^{\text {"334 }}$.

\section{Der Sperrmarkkomplex und die Libka-Mark}

Im Verlauf der Währungskrise des Jahres 1931 waren die RM-Guthaben ausländischer Gläubiger gesperrt worden. Über diese Guthaben und ihre Erträgnisse konnte nur eingeschränkt verfügt werden: Sie konnten weder ins Ausland transferiert noch im Inland allen Verwendungen zugeführt werden. Wenn auch die BdL der Liberalisierung der Kapitalerträgnisse anfänglich zurückhaltend gegen-

332 Vgl. BBK, B 330/2056, Direktorium, Protokoll, 28.9.1954; BBK, B 330/3241, BDI, Memorandum DM-Ausländerkonten, 19.1.1955; Boden (BDI) an Vocke, 20.1.1955; BdL an Erhard, 20.10.1955; BdL an Boden, 10.2.1955. Zu dem besonders umstrittenen Spanien-Fall vgl. BBK, B 330/80, ZBR, Protokoll und Stenogramm, 22.9. 1954; BBK, B 330/2021, BdL an Erhard, 29.9. 1954; BBK, B 330/ 2011, Abs an Vocke, 13.11.1954; Vocke an Abs, 22.11.1954. Vgl. auch BoE, G 1/98, Cobbold, Notes on BIS talks, 10.1.1955.

333 Stand Januar 1954 und Dezember 1956. Vgl. BdL, Monatsbericht März 1955, S. 76; BdL, Monatsbericht Januar 1957, S. 112.

${ }^{334}$ BoE, OV 34/68, Rootham an Bolton, Germany, 6.12.1954. 
überstand und die Konvertibilität nicht auf den Kapitalverkehr ausdehnen wollte, so sah sie den Abbau des sogenannten Sperrmarkkomplexes doch als einen wichtigen Baustein bei der Lockerung der Devisenbewirtschaftung an. Seit Sommer 1953 kreisten die Gedanken der BdL um die Lösung dieses Problems, dem höchste Priorität zugemessen wurde. Immer wenn das Thema der Konvertibilität angesprochen wurde, betonte Vocke: „Indessen sollten wir uns bewußt halten, daß wir zur Errichtung der sogenannten Konvertibilität oder gesunden Währung in erster Linie das gewaltige Problem der Auftauung der Sperrmark vor uns haben, das ganz außerordentliche Anstrengungen auf dem Devisengebiet von uns erfordert. Darauf sollten wir uns konzentrieren. ${ }^{\text {“335 }}$

Die „Auftauung“ der Sperrmark wurde in der BdL zwar als erwünscht, aber gleichzeitig auch als problematisch angesehen. Immerhin hatten sich im Verlauf der Jahre Sperrmarkguthaben in Höhe von 774,3 Mio. DM angesammelt. Davon entfielen 234,6 Mio. DM oder 30,3 Prozent auf den Dollarraum ${ }^{336}$. Wenngleich die vollständige Freigabe der Sperrmark bei einer konvertiblen Währungsreserve von 1169,2 Mio. Dollar den Reservenbestand maximal um 15,8 Prozent hätte verringern können ${ }^{337}$, so hielt doch Vocke eine Freigabe des Kapitals an Sperrmarkgläubiger für „zurzeit völlig indiskutabel“"338. Zwar strebte er für die Zukunft „eine großzügige und wirklich liberale Regelung“ an, die auch die Liberalisierung gewisser Sperrmarkerträgnisse in den Dollarraum vorsah, doch sollte vorerst noch abgewartet werden ${ }^{339}$. Diese Meinung fand bis Ende 1953 regen $\mathrm{Zu}$ spruch innerhalb der BdL. Wenngleich die Dollarüberschüsse Ende des Jahres hoch waren, malte Wolf das Gespenst eines Rückzuges der amerikanischen Truppen aus der Bundesrepublik an die Wand und wies auf die Möglichkeit einer Krise in den Vereinigten Staaten hin. Das wäre unmittelbar mit einem Wiederauftauchen der Dollarlücke verbunden ${ }^{340}$. Da gleichzeitig davon abgeraten wurde, nur einen Erträgnistransfer in den EZU-Raum anzuvisieren, schreckte man vorerst vor Maßnahmen zurück. $\mathrm{Zu}$ dieser Möglichkeit, die zwar angesprochen worden war, meinte Vocke nur: „Es hat gar keinen Sinn, innerhalb der EZU mit diesem Erträgnistransfer anzufangen und gegenüber dem Dollar zu diskriminieren, denn daß wir innerhalb der EZU der stärkste Gläubiger mit der stärksten Währung sind, das wissen die Leute sowieso." ${ }^{341}$

Die ablehnende Haltung der BdL lockerte sich aber schon Anfang des Jahres 1954. Angesichts des „besonders hohen Devisenüberschusses“ im Dezember 1953 sowohl gegenüber dem Dollar- als auch dem EZU-Raum, der möglicherwei-

\footnotetext{
335 BBK, B 330/2012, Vocke an Erhard, 13.11.1953.

336 Stand Ende 1953. Vgl. BdL, Geschäftsbericht 1953, S. 96.

${ }^{337} \mathrm{Da}$ die Sperrmark im Ausland frei handelbar war, konnten theoretisch alle Sperrmarkverpflichtungen zu Dollarverpflichtungen werden.

338 BBK, B 330/3379, Vocke, Zur Frage der Konvertibilität, 9.12.1953.

339 Ebenda.

340 Vgl. BBK, B 330/3379, Wolf, Vermerk über das Konvertibilitätsproblem und die Sperrmarkfrage, 7.12.1953.

${ }^{341}$ BBK, B 330/74, ZBR, Protokoll und Stenogramm, 16.12.1953.
} 
se drohenden OEEC-Empfehlung zur Exportdrosselung und des steten OEECDruckes auf Liberalisierung der Kapitalerträgnisse billigte die BdL nun eine Liberalisierung, die nicht nur Erträgnisse, sondern auch die teilweise Freigabe von $\mathrm{Ka}$ pital einschloß. Damit bezog sie Opposition zum zögernden Finanzministerium. Emminger wies dessen Bedenken mit dem Hinweis auf die Entwicklung der EZU-Position zurück: „Diese Tatsache (die anhaltende Tendenz zu EZU-Überschüssen, M.D.) wird nicht nur den von der OEEC in Paris ausgehenden Druck zu weiteren Liberalisierungs- und sonstigen Entlastungsmaßnahmen verstärken, sondern zwingt uns auch im eigensten Interesse zu einer erneuten Überprüfung der gesamten Transferregelungen. Es wäre bei der gegenwärtigen Entwicklung kaum mehr zu vertreten, wenn wir auf der einen Seite immer weitere Devisenüberschüsse in niedrig verzinslicher und zum Teil sogar unerwünschter Form ansammelten, mit der Folge, daß unsere Ausfuhr auf immer größere Hindernisse stößt und vielleicht sogar eines Tages durch bewußte Maßnahmen gedrosselt werden muß, während sich auf der anderen Seite durch den Nicht-Transfer von Erträgnissen auf ausländische Anlagen im Bundesgebiet unsere Auslandsverschuldung (in zum Teil hochverzinslicher Form) immer weiter erhöhen [. . .] würde. “342

Da das Plädoyer der Bank Anfang 1954 auch die Unterstützung von Abs fand, zog der Finanzminister seine Bedenken Ende Januar zurück ${ }^{343}$. Es wurde erlaubt, orginäre Sperrmarkguthaben, die am 31. Dezember 1953 bestanden, bis zu einer Höhe von 10000 DM in einer Summe bzw. bis zu einer Höhe von 500 DM im Monat zu transferieren ${ }^{344}$. Im weiteren Verlauf wurden auch diese Beschränkungen aufgehoben. Allerdings wurde der Transfer jetzt nur noch in den mit den Beko-Mark-Konten geschaffenen Verrechnungsraum gestattet. Das anfängliche Plädoyer der BdL, auch einen Transfer in den Dollarraum zu genehmigen, wurde wegen der Entwicklung des EZU-Saldos, wegen des kontinuierlichen Drukkes der OEEC und wegen der positiven Haltung der USA zu einer solchen Diskriminierung fallengelassen ${ }^{345}$ : Anfang April 1954 konnten dementsprechend alle Sperrmarkguthaben, die am 31. März 1954 bestanden hatten, auf Beko-MarkKonten übertragen werden. Im September 1954 wurde diese Regelung erweitert, und alle, auch die zukünftigen, Sperrmarkguthaben wurden zum Transfer auf Beko-Mark-Konten zugelassen. Gleichzeitig wurde die Sperrmark in Liberalisierte Kapitalmark bzw. Libka-Mark umbenannt, wobei die Verwendungsmöglichkeiten für Libka-Guthaben erweitert wurden ${ }^{346}$. Ihnen standen nunmehr alle

${ }^{342}$ BBK, B 330/2043, Emminger, Entwurf eines Schreibens an Schäffer, 8.1.1954.

${ }^{343}$ Vgl. BBK, B 330/2011, Abs an Schäffer, 16.1.1954. Die Entscheidung fiel in der Sitzung des Kabinettsausschusses am 25.1.1954. Das Protokoll der entsprechenden Sitzung konnte nicht eingesehen werden, da das Bundeskanzleramt den Bestand nicht freigegeben hat.

344 Vgl. Kühne, Regelungen, S.312 f.

345 Vgl. BBK, B 330/2015, Vocke an Blücher, 31.3.1954; BBK, B 330/2033, Vocke an von Mangoldt, 31.3.1954; BBK, B 330/76, ZBR, Protokoll und Stenogramm, 31.3.1954.

346 Vgl. Kühne, Regelungen, S. 409ff.; BBK, B 330/79, ZBR, Protokoll, 8. 9.1954; BBK, B 330/2021, VA 14, Protokoll über die Ressortbesprechung, 10.9.1954; BBK, NL Emminger, Konvertibilitätskonferenz, Emminger, Bemerkungen, 20.8.1954. 
Tabelle 10: Sperr- und Libka-Mark 1954-1957

\begin{tabular}{|c|c|c|c|c|c|c|c|c|}
\hline & 1954 & & 1955 & & 1956 & & 1957 & \\
\hline & I & III & I & III & I & III & I & III \\
\hline Sperrmark & 778 & 148 & 35 & 11 & 3 & 2 & 2 & 2 \\
\hline Libka-Mark & 0 & 426 & 385 & 427 & 375 & 450 & 497 & 499 \\
\hline Total & 778 & 574 & 420 & 438 & 378 & 452 & 499 & 501 \\
\hline
\end{tabular}

Anmerkung: Alle Angaben in Mio. DM.

Quelle: BdL, Monatsberichte, verschiedene Ausgaben.

Verwendungen innerhalb der Bundesrepublik offen. Allerdings wurde in diesem Zusammenhang die Speisung der Libka-Konten durch Einbringung von Devisen untersagt, womit ein Kontrollinstrument für ausländische Investitionen eingerichtet wurde. Das lag der BdL am Herzen, denn im Herbst 1954 schätzte sie die deutsche Zahlungsbilanzsituation bereits so ein, daß der Zufluß von Auslandskapital schon bald währungspolitisch gefährlich werden könnte und demnach zu kontrollieren sei ${ }^{347}$. Das Direktoriumsmitglied Heinrich Hartlieb führte aus, daß die BdL keine neue Auslandsverschuldung eingehen, sondern den Schuldenkomplex konsolidieren wolle ${ }^{348}$.

Als Konsequenz dieser restriktiven Politik blieb die Nachfrage nach Sperrbzw. Libka-Mark kräftig. Trotz der Verfügungsmöglichkeiten wurden die Guthaben nur eingeschränkt transferiert. Vielmehr wurden die Erträgnisse, wie Tabelle 10 zeigt, zum Teil den Libka-Konten zugeführt. Für die große Nachfrage spricht auch, daß die Libka-Mark zeitweise auf dem Zürcher Devisenmarkt mit einem Agio gehandelt wurde. Von einer „Devisenzwangswirtschaft" konnte also in bezug auf den Kapitalverkehr nicht mehr die Rede sein.

\section{Die Bank deutscher Länder zwischen Europa und der Welt}

Im Sommer 1955 war der deutsche Außenhandel weitgehend von quantitativen Beschränkungen befreit und die deutsche Devisenbewirtschaftung erheblich gelockert: Der Liberalisierungssatz zum OEEC-Raum betrug 91,3 Prozent, der zum Dollarraum 68,1 Prozent. Die bilateralen Zahlungsabkommen waren im Abbau begriffen, und die Sperrmark war der Libka-Mark gewichen. In bezug auf das europäische oder internationale Währungssystem hatten sich die OEECMitglieder auf die Beibehaltung ihrer Zusammenarbeit, den Fortbestand der OEEC-Handelsregeln und des EZU-Zahlungsmechanismus verständigt. Dabei

\footnotetext{
347 Vgl. BAK, B 136/1306, Kabinettsausschuß, Zum 6. OEEC-Bericht, 23.7.1954; BAK, B 102/ 12656, Ehm, Aufzeichnung über die Sitzung des Ausschusses für neue ausländische Devisenanlagen, 16.8.1954; BBK, B 330/2056, Direktorium, Protokoll, 14.9.1954. Vgl. auch Dernburg, Blocked Mark, S. 30 .

348 Vgl. BBK, B 330/79, ZBR, Stenogramm, 11.8.1954.
} 
hatten sie dieses System durch die Härtung der EZU der Konvertibilität und damit dem Bretton-Woods-System angenähert. Gleichzeitig hatten sie das EZU-System mit diesen Modifikationen gläubiger-freundlicher gestaltet. Ferner hatten sie sich geeinigt, das europäische Währungssystem später mit einem gemeinsamen Übergang zur Konvertibilität aufzulösen. Für die Zeit nach der Konvertibilitätserklärung hatten sie mit dem EWA einen europäischen Kredit- und Zahlungsmechanismus verabschiedet. Dieser sollte den europäischen Zusammenhalt sowie die währungspolitische Kooperation Europas auch unter einem Konvertibilitätsregime garantieren.

Obwohl sich Mitte der fünfziger Jahre Schwächen des alten wie des neuen Systems - geringer Einfluß des EZU-Direktoriums, mangelhafte Durchsetzung der Liberalisierungsverpflichtungen, die Möglichkeit unliebsamer, internationaler Kapitalbewegungen und Spekulation - andeuteten oder gar offensichtlich waren, fand dieses neue System die Zustimmung der BdL. Sie hatte sogar in erheblichem Ausmaß an seiner Konstruktion mitgewirkt.

Ihre anfänglich ausgesprochen widersprüchliche Haltung zur Konvertibilitätsfrage - Skepsis gegenüber der Konvertibilität, prinzipielle Kritik am GoldDevisen-Standard und Ablehnung gegenüber den Konvertibilitätsbestrebungen einerseits; Befürwortung der Konvertibilität andererseits - verwandelte sich in den folgenden Jahren in ein eindeutiges Plädoyer für die EZU und den Regionalismus. Obwohl sich die deutsche Reserven- und Außenhandelsposition in diesen Jahren entscheidend verbessert hatte, befürwortete sie - vor die Wahl zwischen Europa und der Welt gestellt - eindeutig die erste Option. Wenn sich auch die Kosten des Regionalismus und der Nicht-Konvertibilität mit den wachsenden deutschen Zahlungsbilanzüberschüssen erhöhten, trat sie doch für den Erhalt der europäischen Währungskooperation ein. Da den Kosten Vorteile, wie zum Beispiel die OEEC-Liberalisierung und damit ein relativ sicherer und möglicherweise zu beeinflussender Exportmarkt sowie eine hohe Verwendbarkeit der EZU-Guthaben, gegenüberstanden, war sie bereit, diese Kosten zu tragen.

Die BdL konnte ihre Position in den internationalen Konvertibilitätsverhandlungen weitgehend durchsetzen, denn sie befand sich im Einvernehmen mit den wirtschaftspolitischen und politischen deutschen Ressorts. Auch fand sie in den anderen EZU-Mitgliedern und in den USA Verbündete. Des weiteren kam ihr ihre Machtposition als Kreditgeberin der EZU bzw. als Zentralbank eines Gläubigerlandes zu Hilfe. Aufgrund dieser Machtposition konnte sie zu einem bedeutenden Teil die Verhandlungen über die EZU und das EWA bestimmen. Dagegen erlitten das Vereinigte Königreich und der deutsche Wirtschaftsminister Erhard, welche die Abschaffung der EZU und die Konvertibilität befürworteten, eine Niederlage. Wenngleich sie mit ihren Vorstößen und Plänen, worauf die BdL oftmals nur reagieren konnte, die Tagesordnung bestimmten, mußten sie letztendlich angesichts der geschlossenen Opposition gegenüber derartigen Plänen und angesichts der britischen außenwirtschaftlichen Schwäche einlenken. 
Das Bild einer BdL, die den Regionalismus und die EZU befürwortete, ist allerdings nicht eindeutig. Es ist auch festzuhalten, daß die BdL bei vielen Gelegenheiten für eine Härtung der EZU eintrat, Lockerungen der Devisenbewirtschaftung verfügte und sich durch unilitaterale Maßnahmen der Konvertibilität annäherte. Ein Großteil dieser Politik kann darauf zurückgeführt werden, daß ihr - neben dem Abbau des Bilateralismus und der Regelung des SperrmarkKomplexes - an einer Good Creditor Policy lag. Mit diesen Maßnahmen strebte sie ein besseres Funktionieren der EZU an. Darüber hinaus war zum Beispiel die Dollarliberalisierung auf das Drängen der USA und die hohen deutschen Devisenreserven zurückzuführen. Ferner wurde mit dem Andauern der Konvertibilitätsverhandlungen mehr und mehr offensichtlich, daß die EZU nicht fortbestehen würde. Schließlich ist festzuhalten, daß mit der verbesserten europäischen Reservenposition die Konvertibilität die europäische Liberalisierung nicht mehr akut gefährdete.

In den Jahren 1952 bis 1955 war die BdL in bezug auf die Konstruktion eines internationalen Währungssystems eine wichtige und entscheidende Instanz. Die Tatsache, daß sie bei den internationalen Konvertibilitätsverhandlungen nicht immer von innen- und außenpolitischen Umständen absehen konnte, und die Tatsache, daß sie zum Teil eine Gefangene der positiven deutschen Außenhandels- und Reservenentwicklung war, verändert dieses Bild nur geringfügig. 